\title{
Algorithmic equiresolution of deformations of embedded varieties
}

Augusto Nobile

\begin{abstract}
A theory of simultaneous resolution of singularities for families of embedded varieties (over a field of characteristic zero) parametrized by the spectrum of a suitable artinian ring, and compatible with a given algorithm of resolution, is presented. As usually, this a simple consequence of a similar theory for analogous families of basic objects, to which the main portion of this article is devoted.
\end{abstract}

\section{Introduction}

The fact that an arbitrary algebraic variety $X$, over a field of characteristic zero, admits a resolution (that is, a proper birational morphism $f: X^{\prime} \rightarrow X$, with $X^{\prime}$ regular) was first established by H. Hironaka in his celebrated 1964 article [14].

Once resolution is available for a single variety, a natural question is to try to simultaneously resolve all the members of a given family, say parameterized by a variety $T$, a process that might be called equiresolution. It is clear that this is not always possible: to succeed means that, in some sense, the singularities of the different members are not too different. Moreover, there are many conditions that can be imposed to a simultaneous resolution process, which lead to different notions (see [25], written in the late 1970's). Another possible approach, using more recent developments, involves algorithmic resolutions. These theories arose as an attempt to improve and better understand the article [14].

In [14] the resolution is achieved by means of a finite sequence of blowingups, or monoidal transforms, each with a regular center contained in the

2000 Mathematics Subject Classification: 14B05, 14E15, 14E99,14D99.

Keywords: Resolution of singularities, simultaneous resolution, deformation. 
singular set (and satisfying some further technical conditions, like normal flatness). But the proof, aside from being very complicated, is existential in the sense that it is not made very clear how to choose each center. For many years there was no improvement upon the original presentation. But starting in the late 1980's several authors (basing their work, in part, on some pioneering contributions of Hironaka himself, e.g., [15]) obtained "algorithmic resolution theorems", i.e., processes of resolution where one specifies the center to choose each time we blow-up. See, e.g., [26], [11], [5], [6], [9], [17], [28]. This approach not only simplifies the original proof of [14], but affords some additional results. For instance, one obtains equivariant resolutions, i.e., compatible with the action of a group on the variety.

A common feature of all these works is the substitution of the original problem, where one directly deals with algebraic varieties, by another, seemingly more technical one, where one tries to "improve" other objects, involving a sheaf of ideals on a regular ambient scheme. More precisely, one deals with basic objects. We follow the terminology of [5], although the language and presentation vary with the different authors. A basic object (over a field $k$ ) is a four-tuple $B=(W, I, b, E)$ where $W$ is a $k$-smooth variety, $I$ a never-zero sheaf of $\mathcal{O}_{W}$-ideals, $b$ a positive integer and $E$ a collection of smooth divisors of $W$ with normal crossings. One defines a suitable notion of permissible transformation, which gives us a new basic object, induced by $B$ on the blowing-up $W^{\prime}$ of $W$ with an appropriate regular center. Then essentially the goal is to reach, by means of a sequence of permissible transformations, a basic object $B_{r}=\left(W_{r}, I_{r}, b, E_{r}\right)$ where for all $x \in W_{r}$ the order of the stalk $\left(I_{r}\right)_{x}$ in $\mathcal{O}_{W_{r}, x}$ is less than $b$. Moreover, one should be able to describe each center, say as the set of maximum value of an upper semicontinuous function taking values in a suitable totally ordered set (depending on the dimension of $W$ only). If this is done in an appropriate way, the solution to this problem easily implies that of resolving varieties, in such a way that the centers of the blowing-ups used are explicitly described ([5], Section 5). See also, for other procedures, e.g., [6], [16] or [28].

So, returning to the problem of equiresolution, a possible approach is this one: in the presence of a given algorithm of resolution (say, of embedded varieties, i.e., a closed inclusion of varieties $X \subset W$, with $W$ regular), and given a family of such embedded varieties, introduce and study a reasonable notion of algorithmic equiresolution, i.e., a sequence of monoidal transformations of the ambient total space inducing on each fiber the algorithmic resolution process. When the parameter space is smooth (or, at least, reduced), this problem was studied in [10], where several proposed definitions (for families of ideals and of embedded varieties) are seen to be equivalent. See also [7], Section 6 . 
But there is something unsatisfactory about the restriction to the use of reduced parameter spaces only. In many problems one is naturally lead to the consideration of families parameterized by non-reduced schemes. For instance, assuming a suitable resolution algorithm has been fixed, an application discussed in [10] consists of a stratification of the Hilbert scheme of subvarieties of $\mathbf{P}^{n}$ with a given Hilbert polynomial, expressing it as a union of reduced locally closed subschemes, such that the restriction of the universal family to each stratum is algorithmically equisolvable, and universal with respect to equisolvable families parameterized by reduced schemes. Clearly the "reduced" condition is not entirely satisfactory. Indeed, the Hilbert scheme itself might be non-reduced, moreover many usual techniques to study features such as smoothness, tangent spaces, etc., involve the consideration of families parametrized by schemes of the form $S=\operatorname{Spec}(A)$, with $A$ an artinian ring. So, it seems natural to study, at least, the notion of algorithmic equiresolution for families parametrized by $S$ as above (i.e., infinitesimal families or infinitesimal deformations of embedded varieties).

In this paper the latter problem is investigated. As said, initially we deal with basic objects over an artinian ring $A$ as above (also called $A$ or $S$-basic objects, see Definition 4.1). Of course, the main difficulty here is that we have just one fiber (the naturally induced basic object over the only point of $S$ ), so that to directly compare the algorithmic resolutions of the various fibers makes no sense. Rather, one should try to introduce conditions that make the notion "the algorithmic resolution of the (single) fiber evenly spreads along $S=\operatorname{Spec}(A)$ " rigorous. It seems that, essentially, the only tool we have is to require that, in a suitable sense and working with appropriate completions of local rings, the orders of certain series do not change when we reduce the coefficients modulo the maximal ideal of $A$. This is what we attempt to do.

For instance, the most fundamental notion developed along these lines is that of permissible $B$-center (or permissible center relative to $S$ ), where $B=$ $(p: W \rightarrow S, I, b, E),(S=\operatorname{Spec}(A), A$ an artinian local ring whose residue field has characteristic zero and $p$ is a smooth morphism) is a basic object over $A$, see 4.1 for the complete definition. Namely, by controlling certain orders of ideals, one may impose a condition on a closed subscheme $C \subset W$, flat over $S$ which, were $S$ a reduced scheme rather than an infinitesimal one, would induce on each fiber of $p$ (a regular variety over a field) a permissible center, say in the sense of [5]. Then it is possible to define a natural notion of permissible transform of $B$ with center $C$. If we repeat the process of choosing a permissible center and transforming, we get what we call an A-permissible sequence of $A$-basic objects, and this induces a permissible sequence (in the sense of [5]) at the level of closed fibers. If this sequence is 
a resolution of $B^{(0)}$ (the fiber of $B$ ) we say that our $A$-permissible sequence is an equiresolution of the $A$-basic object $B$. (This is done in Sections 3 and 4). A natural question is: if an algorithm for resolution of basic objects over fields (of characteristic zero) is given, when will an equiresolution be algorithmic?

In [10] the problem of algorithmic equiresolution of families parametrized by a reduced scheme is studied. There one works with an arbitrary resolution algorithm satisfying certain conditions ("good algorithms"). So far I do not know how to treat the present deformation situation with this degree of generality. Trying to get experience, I deal with a specific resolution algorithm, namely essentially that of [11] (or [5], [21], [8]). We review the algorithm, for the reader's convenience, in Section 2.

Let us better explain what is our main result. Let $\mathcal{A}$ be the class of artinian local algebras, whose residue a field has characteristic zero. If $B^{(0)}$ is a basic object over a characteristic zero field $k$, write $\ell(B)=r$ if

$$
\text { (1) } \quad B^{(0)}=B_{0}{ }^{(0)} \leftarrow \cdots \leftarrow B_{r}{ }^{(0)}
$$

is the algorithnmic resolution of $B^{(0)}$. Then we have:

Theorem 1.1. There is a function associating to each A-basic object

$$
B=(W \rightarrow \operatorname{Spec} A, I, b, E)
$$

$(A \in \mathcal{A})$ with fiber $B^{(0)}$ a non-negative integer $e(B) \leq \ell\left(B^{(0)}\right)$ and $A$ permissible centers $C_{0}, \ldots, C_{e(B)-1}$, where $C_{0}$ is a $B=B_{0}$-center, $C_{1}$ an $A$-center for the transform $B_{1}$ of $B_{0}$ with center $C_{0}$, and so on (thus an A-permissible sequence

$$
\text { (2) } \quad B=B_{0} \leftarrow B_{1} \leftarrow \cdots \leftarrow B_{e(B)}
$$

is determined), such that: $(i)$ each center $C_{i}$ induces on the fiber the $i$ th algorithmic center used in the sequence (1) (and hence (2) induces the truncation at level $e(B)$ of $(1))$, (ii) this correspondence is functorial with respect to homomorphisms $A \rightarrow A^{\prime}$ and etale morphisms $W^{\prime} \rightarrow W$.

The sequence (2) thus obtained is called the partial algorithmic equiresolution of $B$. When $e(B)=\ell\left(B^{(0)}\right)$ we say that $B$ is algorithmically equisolvable and call the corresponding sequence (2) its algorithmic equiresolution.

The idea to show this is to try to generalize, or "spread", the different steps of the algorithmic resolution process of the fiber (a basic object over a field) to the $A$-basic object $B$. The number $e(B)$ indicates how far we can go in the process. Of course, often $e(B)<\ell\left(B^{(0)}\right)$, not any infinitesimal family 
will be equisolvable. This generalization is studied in Sections 4 though 8 . Theorem 1.1 is proved in Section 9, where we introduce conditions which, if valid, insure that centers used in the algorithmic resolution of the fiber $B^{(0)}$ extend over $S=\operatorname{Spec}(A)$, producing the unique centers $C_{i}$ of the Theorem.

From this theorem similar results for infinitesimal families of ideals or of embedded varieties may be easily obtained.

Actually, the algorithm we use is not strictly that of [11]. We use a slight variation of this method. The reason is that there are some technical difficulties to adapt the whole process of [11] to our situation. This occurs at a key inductive step, where a basic object $B=(W, I, b, E)$ is replaced by another $B_{Z}$, whose underlying scheme is a regular hypersurface $Z$ of $W$, which is only locally defined, not canonically (what many authors call a $h y$ persurface of maximal contact). Because of this, there is a serious glueing problem to get globally valid statements. In the references above this is addressed by re-doing the theory in a more general set-up, that of generalized basic objects where, after proving some technical results, the glueing process is easier. It seems difficult to adapt this approach to the context of basic objects over an artinian ring $A$. But, following a suggestion of S. Encinas, by using techniques of Wlodarczyk in [28] (homogenized basic objects, specially a suitable version of his "Glueing Lemma"), it is possible to develop a satisfactory theory over $A$, bypassing the use of generalized basic objects. See 9.11 for more details.

Most of this article deals with basic objects, the transition to families of ideals or embedded varieties, by now standard (see [5], Section 5), is explained in Section 9. Of course, geometrically the most interesting case is that of families of varieties, but at present it does not seem possible to discuss this situation without a rather lengthy previous study of families of basic objects (or something similar). Also at the end of Section 9 it is indicated how our conditions $\mathcal{E}_{i}$ afford a natural notion of algorithmic equiresolution for families parametrized by arbitrary noetherian schemes.

In another article we shall compare this notion with others available when the parameter space is reduced $([10])$. In the future we hope to study applications (like those discussed in [10], Section 4) as well as the functors on Artin rings that naturally arise (see [24]).

In an appendix (Section 11) we include some rather basic algebraic results, that we could not find in the literature.

It is my pleasure to thank O. Villamayor and S. Encinas for their help and encouragement, as well as the referee, for useful suggestions to improve this article. 


\section{The algorithm}

2.1. Terminology and conventions. In general, we shall use the notation and language of [13]. We describe next a few exceptions. If $W$ is a scheme, a $W$-ideal will mean a coherent sheaf of $\mathcal{O}_{W}$-ideals. If $I$ is a $W$-ideal, the symbol $\mathcal{V}(I)$ will denote the closed subscheme of $W$ defined by $I$. As usual, $V(I)$ will denote the closed subset of the underlying topological space of $W$ of zeroes of $I$. If $Y$ is a closed subscheme of a scheme $W$, the symbol $I(Y)$ denotes the $W$-ideal defining $Y$. An algebraic variety over a field $k$ will be a reduced algebraic $k$-scheme. If $W$ is a reduced scheme, a never-zero $W$-ideal is a $W$-ideal $I$ such that the stalk $I_{x}$ is not zero for all $x \in W$, in general $I$ is a never-zero ideal of $W$ if $I \mathcal{O}_{W^{\prime}}$ is never-zero, with $W^{\prime}=W_{\text {red }}$.

The term local ring will mean noetherian local ring. In general, the maximal ideal, or radical, of a local ring $R$ will be denoted by $r(R)$. Often, we write $(R, M)$ to denote the local ring $R$ with maximal ideal $M$. The order of an ideal $I$ in the local ring $(A, M)$ is the largest integer $s$ such that $I \subseteq M^{s}$. If $W$ is a noetherian scheme, $I$ is a $W$-ideal and $x \in W$, then $\nu_{x}(I)$ denotes the order of the ideal $I_{x}$ of $\mathcal{O}_{W, x}$.

All the varieties we consider in this paper are defined over characteristic zero fields.

2.2. The goal of this article is to study certain questions on simultaneous resolution, or equiresolution, in the presence of an algorithm of resolution of singularities. The most interesting case is that of algebraic varieties, but in our approach it is more convenient to study first, in detail, the analogous problem for a more formal type of objects, the so-called basic objects (over fields). From this similar results for varieties easily follow. We shall work with a specific algorithm, essentially that developed in [26] and [27] (and presented more sistematically in [11] or [5]). For the reader's convenience, and also to motivate our work in the succeeding sections, we shall briefly review this algorithm. We omit proofs and many details, found in the references just mentioned or, extended to the more general setting of basic objects over artinian rings, in further sections of the present paper.

In this section we work with algebraic schemes over a field $k$, of characteristic zero (although something similar can be done in a more general context, see $[10,5.11])$.

2.3. A basic object (over a field $k$, of characteristic zero) is a four-tuple $B=(W, I, b, E)$, where $W$ is a smooth, equidimensional algebraic variety over $k, I$ is a never-zero $W$-ideal, $b>0$ is an integer and $E=\left(H_{1}, \ldots, H_{m}\right)$ is a sequence of distinct regular hypersurfaces of $W$ (i.e., each $H_{i}$ is a regular Weil divisor of $W$ ) with normal crossings $([5,2.1])$. The smooth variety $W$ 
is the underlying scheme of $B$, denoted by us $(B)$. The dimension of $B$ is the dimension of the variety us $(B)$.

In [11] or [5], the notation $(W,(I, b), E)$ is used for a basic object, we drop the inside parenthesis to simplify.

2.4. The singular set $\operatorname{Sing}(B)$ of a basic object is $\left\{x \in W: \nu_{x}(I) \geq b\right\}$. This is a closed set of $W$. Indeed, one may introduce an operation $\Delta^{i}$ on $W$-ideals, $i \geq 1$, so that $\operatorname{Sing}(B)=V\left(\Delta^{b-1}(I)\right)$. Concerning $\Delta=\Delta^{1}$, if $w$ is a closed point of $W$ and $x_{1}, \ldots, x_{r}$ is a regular system of parameters of $R=$ $\mathcal{O}_{W, w}$ and $D_{i}$ is the derivation associated to $x_{i}$ (the "partial derivative" with respect to $x_{i}$ ), then $\Delta(I)_{w}$ is the ideal of $R$ generated by $I_{w} \cup\left\{D_{i} f: f \in I_{w}\right\}$, $\Delta^{i}$ is defined by iteration. One defines $\Delta(I)$ more intrinsically by means of suitable Fitting ideals of $\Omega_{\nu(I) / k}, k$ the base field. See [5, Section 13] for more details.

The use of differential methods (such as the operators $\Delta^{j}(I)$ ) was pioneered by Jean Giraud (see [12]). These have greatly helped to clarify and simplify the theory of resolution of singularities. They give us an alternative way to treat Hironaka's notion of maximal contact, and play and important role in algorithmic techniques.

2.5. Permissible transformations. If $B=(W, I, b, E), E=\left(H_{1}, \ldots, H_{m}\right)$ is a basic object, a permissible center for $B$ is a closed subscheme $C \subset W$ having normal crossings with the hypersurfaces $H_{i}, i=1, \ldots, m$ (in particular, $C$ is regular) such that $C \subseteq \operatorname{Sing}(B)$. We define the transform of $B$ with center $C$ as the basic object $\left.B_{1}=\left(W_{1}, I_{1}, b, E_{1}\right)\right)$ where $W_{1}$ is the blowingup of $W$ with center $C$ (so, we have a natural morphism $W_{1} \rightarrow W$ ), $E^{\prime}=$ $\left(H_{1}^{\prime}, \ldots, H_{m}^{\prime}, H_{m+1}\right)$, where $H_{i}^{\prime}$ is the strict transform of $H_{i}(i=1, \ldots m)$ and $H_{m+1}$ is the exceptional divisor, finally $I_{1}:=I\left(H_{m+1}\right)^{-b} I \mathcal{O}_{W_{1}}$ (cf. [5], Section 3). The process of replacing $B$ by such new basic object $B_{1}$ is is called the (permissible) transformation of $B$ with center $C$, denoted by $B \leftarrow B_{1}$. We'll also write $B_{1}:=\mathcal{T}(B, C)$.

If $W_{1} \rightarrow W$ is as above, we also define the proper transform of $I$ to $W_{1}$ as the $W_{1}$-ideal $\mathcal{E}^{-a} I \mathcal{O}_{W_{1}}$, where $\mathcal{E}$ defines the exceptional divisor and the exponent $a$ is as large as possible. This integer is constant along each irreducible component of the center $C$ used, but in general not globally constant.

2.6. A resolution of the basic object $B$ is a sequence $B_{0} \leftarrow \cdots \leftarrow B_{r}$, where each arrow $B_{j} \leftarrow B_{j+1}$ is a permissible transformation, such that $\operatorname{Sing}\left(B_{r}\right)=\emptyset$.

An algorithm of resolution (for basic objects in $\mathcal{S}$ ) is a rule that associates to each positive integer $d$ a totally ordered set $\Lambda^{(d)}$ and, for any given 
basic object $B_{0}=\left(W_{0}, I_{0}, b_{0}, E_{0}\right)$ of dimension $d$, functions as follows. First, there is an upper semicontinuous function $g_{0}: \operatorname{Sing}\left(B_{0}\right) \rightarrow \Lambda^{(d)}$, (taking finitely many values), such that $C_{0}=\operatorname{Max}\left(g_{0}\right)=\left\{w \in \operatorname{Sing}\left(B_{0}\right): g_{0}(w)\right.$ is maximum $\}$ is a permissible center. If $B_{1}=\mathcal{T}\left(B_{0}, C_{0}\right)$ has $\operatorname{Sing}\left(B_{1}\right) \neq \emptyset$, a function $g_{1}: \operatorname{Sing}\left(B_{1}\right) \rightarrow \Lambda^{(d)}$ is given, such that $C_{1}=\operatorname{Max}\left(g_{1}\right)$ is a well determined $B_{1}$-permissible center, which we blow-up, and so on. Eventually we get in this way a permissible sequence $B_{0} \leftarrow B_{1} \leftarrow \cdots \leftarrow B_{r}$. We require that this be a resolution, i.e., $\operatorname{Sing}\left(B_{r}\right)=\emptyset$. This is called the algorithmic resolution sequence of $B$. Moreover, this should be stable under etale base change, meaning that if $B^{\prime}$ is the basic object obtained by pull-back under an etale map $W^{\prime} \rightarrow W$, then the pull-back of the resolution sequence above may be identified to the resolution sequence of $B^{\prime}$ (and the new resolution functions are induced by the original ones) and, similarly, under extension of the base field.

2.7. In this paper we shall work with a specific algorithm, a variant of that of [5] or [11] (with some elements from [28]). We shall refer to it as the VW-algorithm. In its construction, the following auxiliary notions are important.

(i) the functions $\omega_{r}$ and $t_{r}$. If

$$
B_{0} \leftarrow \cdots \leftarrow B_{r}
$$

is a sequence of basic objects and permissible transformations (where we write $B_{j}=\left(W_{j}, I_{j}, b, E_{j}\right)$, for all $\left.j\right)$, we define, for $x \in \operatorname{Sing}\left(B_{r}\right), \omega_{r}(x):=$ $\nu_{x}\left(\bar{I}_{r}\right) / b$ (where $\bar{I}_{r}$ denotes the proper transform of $I_{0}$ to $W_{r}$ ). It can be proved that if in our sequence the center of each transformation is contained in $\operatorname{Max}\left(\omega_{j}\right)$ (the set of points where $\omega_{j}$ reaches its maximum value $\max \left(\omega_{j}\right)$ ) then $\max \left(\omega_{j-1}\right) \geq \max \left(\omega_{j}\right)$, for $j<r$. Such a sequence will be called $\omega$-permissible.

The functions $t_{r}$ are defined by induction on the length $r$ of a $\omega$-permissible sequence as above. If $r=0$, for $x \in \operatorname{Sing}\left(B_{0}\right)$ we write $t_{0}(x)=$ $\left(\omega_{0}(x), n_{0}(x)\right)$, where $n_{0}(x)$ is the number of hypersurfaces in $E_{0}$ containing $x$. Assume that $t_{j}=\left(\omega_{j}, n_{j}\right)$ was defined (on $\left.\operatorname{Sing}\left(B_{j}\right)\right)$ for $j<r$ and that in our sequence (1) is $t$-permissible, i.e., that each center $C_{i}$ used in the blowing-ups is contained in the subset of $\operatorname{Sing}\left(B_{i}\right)$ where $t_{i}$ reaches its maximum value (in particular then (1) is $\omega$-permissible). Let $s$ be the smallest index such that $\max \left(\omega_{s}\right)=\max \left(\omega_{r}\right)$ and $E_{r}^{-}$the collection of the hypersurfaces in $E_{r}$ which are strict transforms of those in $E_{s}$. Then, for $x \in \operatorname{Sing}\left(B_{r}\right)$ we set: $t_{r}(x)=\left(\omega_{r}(x), n_{r}(x)\right)$, where $n_{r}(x)$ is the number of hypersurfaces in $E_{r}^{-}$containing $x$. A $B_{r}$-center which is contained in $\operatorname{Max}\left(t_{r}\right)$ will be called $t$-permissible. It can be proved that in a $t$-permissible sequence the sequence $\max \left(t_{j}\right)$ is non-increasing. 
(ii) Monomial objects. A basic object $B=(W, I, b, E)$, where $E=$ $\left(H_{1}, \ldots, H_{m}\right)$ is monomial if for each $w \in W$ we have:

$$
I_{w}=I\left(H_{1}\right)^{\alpha_{1}(w)} \ldots I\left(H_{m}\right)^{\alpha_{m}(w)}
$$

with each function $\alpha_{i}: W \rightarrow \mathbf{Z}$ constant on each irreducible component of $H_{i}$ and zero outside $H_{i}$. If $B$ is monomial, one may define (using combinatorial techniques) a function $\Gamma_{B}$ from $\operatorname{Sing}(B)$ to $\mathbf{Z} \times \mathbf{Q} \times \mathbf{Z}^{\mathbf{N}}$ which is uppersemicontinuous (when the target is lexicographically ordered). Then, it turns out that $C:=\operatorname{Max}\left(\Gamma_{B}\right)$ is a permissible center and that, if $B_{1}=\mathcal{T}(B, C)$, $\max \left(\Gamma_{B_{1}}\right)<\max \left(\Gamma_{B}\right)$. This center $C$ is the intersection of certain hypersurfaces in $E$, which are explicitly determined from $\Gamma_{B}$ (see [5, Section 20], or 5.4 of this paper).

2.8. Now we briefly describe our resolution algorithm, that will be called the $V W$-resolution algorithm.

$(\alpha)$ For each integer $d \geq 1$ we must indicate a totally ordered set $\Lambda^{(d)}$ and, for any given basic object $B_{0}=\left(W_{0}, I_{0}, b_{0}, E_{0}\right)$ over $k$ of dimension $d$, the corresponding resolution functions $g_{j}$.

This process will be defined inductively on the dimension of $B_{0}$, as follows. In the sequel, $\mathcal{S}_{1}:=\mathbf{Q} \times \mathbf{Z}$ and $\mathcal{S}_{2}:=\mathbf{Z} \times \mathbf{Q} \times \mathbf{Z}^{\mathbf{N}}$, in all cases lexicographically ordered.

If $\operatorname{dim}\left(B_{0}\right)=1$, let $\Lambda^{(1)}=\mathcal{S}_{1} \cup \mathcal{S}_{2} \cup\left\{\infty_{1}\right\}$, where if $a \in \mathcal{S}_{2}$ and $b \in \mathcal{S}_{1}$ then $a>b$ and $\infty_{1}$ is the largest element of the set. Then we define for $w \in \operatorname{Sing}\left(B_{0}\right), g_{0}(w)=t_{0}(w)$ and, if $g_{i}$ is defined for $i<s$, determining a permissible sequence $B_{0} \leftarrow B_{1} \leftarrow \cdots \leftarrow B_{s}$ we define, for $w \in \operatorname{Sing}\left(B_{s}\right)$, $g_{s}(w)=t_{s}(w)$ if $\omega_{s}(w)>0$ and $g_{s}(w)=\Gamma_{B_{s}}(w)$ otherwise.

In the induction step we need the following auxiliary construction.

$(\beta)$ Inductive step. Assume that we have an algorithm of resolution defined for basic objects of dimension $<d$. Consider a $t$-permissible sequence of basic objects and transformations

$$
B_{0} \leftarrow B_{1} \leftarrow \cdots \leftarrow B_{s}
$$

Let $w \in \operatorname{Max}\left(t_{s}\right)$ and suppose that, near $w, \operatorname{dim} \operatorname{Max}\left(t_{s}\right) \leq d-2$. Then there is an open neighborhood $U$ of $w$ (in $W_{s}=u s\left(B_{s}\right)$ ), a hypersurface $Z_{s}$ on $U$, containing $w$, and a basic object $B_{s}{ }^{*}=\left(Z_{s}, I_{s}{ }^{*}, b_{s}{ }^{*}, E_{s}{ }^{*}\right)$, having the following properties:

(i) $\operatorname{Sing}\left(B_{s}{ }^{*}\right)=\operatorname{Max}\left(t_{s \mid U}\right)$.

(ii) The algorithmic resolution sequence corresponding (by the induction hypothesis) to $B_{s}{ }^{*}$ :

$$
B_{s}^{*} \leftarrow\left(B_{s}^{*}\right)_{1} \leftarrow \cdots \leftarrow\left(B_{s}^{*}\right)_{p}
$$


(determined, say, by resolution functions $\widetilde{g}_{i}$ ) induces a $t$-permissible sequence

$$
\widetilde{B}_{s} \leftarrow \widetilde{B}_{s+1} \leftarrow \widetilde{B}_{s+p}
$$

(obtained by using the same centers $C_{i}=\operatorname{Max}\left(\widetilde{g}_{i}\right)$, and denoting by $\widetilde{B}_{s}$ the restriction of $B_{s}$ to $U$ ).

(iii) If $\max \left(t_{s}\right)=\max \left(t_{s+j}\right)(j=1, \ldots, p)$ then, for all such indices $j$, $u s\left(\left(B_{s}{ }^{*}\right)_{j}\right)$ gets identified to $Z_{s+j}$, the strict transform of $Z_{s}$ to $u s\left(\widetilde{B}_{s+j}\right)$ and $\operatorname{Sing}\left(\left(B_{s}{ }^{*}\right)_{j}\right)=\operatorname{Max}\left(\widetilde{t_{r+j}}\right)$ (where $\widetilde{t_{j}}$ are the $t$-functions of the sequence $(3)$ ).

(iv) Under the assumption of (iii) for all $j=0, \ldots, p$, if $w_{j} \in \operatorname{Max}\left(\widetilde{t_{s+j}}\right)$ is in the pre-image of $w$ (under the morphism $\widetilde{B}_{s+j} \rightarrow \widetilde{B}_{s}$ arising from (3)), the resolution function $\widetilde{g}_{j}$ of $B_{s}{ }^{*}$ defines a function (still denoted by $\widetilde{g}_{j}$ ) on a neighborhood (in $\operatorname{Max}\left(\widetilde{t_{s+j}}\right)$ ) of $w_{j}$. The process does not uniquely determine the neighborhood $U$ nor the hypersurface $Z_{s}$, but the value $\widetilde{g}_{j}\left(w_{j}\right)$ is independent of the choices.

In 2.9 we are going to indicate how to make these constructions.

$(\gamma)$ Now, assuming the resolution functions given for dimension $<d$, we'll define resolution functions $g_{j}$ for objects of dimension $d$ as follows. In this case, the totally ordered set of values will be: $\Lambda^{(d)}=\left(\mathcal{S}_{1} \times \Lambda^{(d-1)}\right) \cup \mathcal{S}_{2} \cup\left\{\infty_{d}\right\}$, where $\mathcal{S}_{1} \times \Lambda^{(d-1)}$ is lexicographically ordered, any element of $\mathcal{S}_{2}$ is larger than any element of $\mathcal{S}_{1} \times \Lambda^{(d-1)}$ and $\infty_{d}$ is the largest element.

Consider first a single basic object $B_{0}$. Let $M:=\operatorname{Max}\left(t_{0}\right)$ and $M(1)$ the union of the one-codimensional components of $M$. Given $x \in \operatorname{Sing}\left(B_{0}\right)$, necessarily we have $\omega_{0}(x)>0$ and there are three cases. (a) $x \in M(1)$. Then set $g_{0}(x)=\left(t_{0}(x), \infty_{d-1}\right)$. (b) $x \in M \backslash M(1)$. Then take a neighborhood $U$ of $x$ (in $W$ ) such that the basic object $B_{0}^{*}$ above is defined and the function $\widetilde{g_{0}}: Z_{0} \rightarrow \Lambda^{(d-1)}$ as in (iv) above (with $s=j=0$ ) Then set $g_{0}(x)=$ $\left(t_{0}(x), \widetilde{g}_{0}(x)\right)$. This value is independent of the choices made. (c) $x \notin M$ Then set $g_{0}(x)=\left(t_{0}(x), \infty_{d-1}\right)$.

Assume now that resolutions functions $g_{i}, i=0, \ldots, j-1$ have been defined, determining centers $C_{i}=\operatorname{Max}\left(g_{i}\right), i=0, \ldots, j-1 \ldots$, leading to a permissible sequence $B_{0} \leftarrow \cdots \leftarrow B_{j}, B_{i}=\left(W_{i}, I_{i}, b, E_{i}\right), i=0, \ldots, j$, $j \geq 0$. We assume that if $B_{j-1}$ is not a monomial object, then this is a $t$-sequence.

There are two basic cases: (a) $\max \left(\omega_{j}\right)=0,(\mathrm{~b}) \max \left(\omega_{j}\right)>0$.

In case (a), $B_{j}$ is monomial, for $x \in \operatorname{Sing}\left(B_{j}\right)$ let $\Gamma_{j}$ be its $\Gamma$-function and set $g_{j}(x):=\Gamma_{j}(x)$. In case (b), let $M_{1}(j)$ denote the union of the one-codimensional components of $M(j):=\operatorname{Max}\left(t_{j}\right)$ and $H$ the exceptional divisor of the blowing-up (with center $C_{i}$ ) $W_{j-1} \leftarrow W_{j}$. For $x \in \operatorname{Sing}\left(B_{j}\right)$ there are three sub-cases: 
$\left(b_{1}\right) x \in M_{1}\left(t_{j}\right) \cap H$. Then we set $g_{j}(x)=\left(t_{j}(x), \infty_{d-1}\right)$ if $x \in M_{1}(j)$ and $g_{j}(x)=\left(t_{j}(x), \infty_{d-1}\right)$ if $x \in \operatorname{Sing}\left(B_{j}\right)$ but $x \notin M_{1}(j)$.

$\left(b_{2}\right) x \in\left(M(j) \backslash M_{1}(j)\right) \cap H$. Then consider the smallest index $s$ such that $t_{s}\left(x_{s}\right)=t_{j}(x)$, where $x_{s}$ is the image of $x$ in $\operatorname{Sing}\left(B_{s}\right)$ induced by the sequence above. Using the construction of $(\beta)$, applied to $x_{s} \in W_{s}$, we obtain resolution functions of $B_{s}^{*}, \widetilde{g_{0}}, \widetilde{g_{1}}, \ldots$ Then it makes sense to take $\widetilde{g_{j-s}}(x)$, and it can be proved that this value is well-defined. We set $g_{j}(x)=\left(t_{j}(x), \widetilde{g_{j-s}}(x)\right) \in \mathcal{S}_{1} \times \Lambda^{(d-1)}$.

$\left(b_{3}\right) x \notin H$. Then, if $x^{\prime}$ is the image of $x$ in $W_{j-1}$, set $g_{j}(x)=g_{j-1}\left(x^{\prime}\right)$

With this definition, if $B_{j}$ is not monomial then the center $C_{j}=\operatorname{Max}\left(g_{j}\right)$ is contained in $\operatorname{Max}\left(t_{j}\right)$.

It can be proved that the sequence $\left\{\max \left(g_{j}\right)\right\}$ is strictly decreasing, which leads to a resolution of $B([5]$ or [11]).

2.9. We shall better explain some details of this process, specially the crucial inductive step of 2.8. For this, we must review some other concepts.

( $\alpha$ ) Adapted hypersurfaces, nice objects. A hypersurface $Z \subset W$ is adapted to $B$ (or $Z$ is $B$-adapted) if the following conditions hold: (A1) $I(Z) \subseteq \Delta^{b-1}(I)$ (an inclusion of sheaves of $\mathcal{O}_{W}$-ideals), (A2) $Z$ is transversal to $E$ (see 6.1). If, moreover: (A3) "Whenever $D$ (resp. $D^{\prime}$ ) is an irreducible component of $Z$ (resp. of $\left.V\left(\Delta^{b-1}(I)\right)\right)$ then $D \neq D^{\prime \prime}$ holds, we say that $Z$ is inductive.

We shall say that $B$ (a basic object in $\mathcal{S}$ ) is nice if either $\operatorname{Sing}(B)$ is empty or $B$ admits an adapted hypersurface. An adapted hypersurface is necessarily regular.

$(\beta)$ Inductive objects If $B$ is a nice basic object we define a $W$-ideal, called the coefficient ideal and denoted by $\mathrm{C}(I)$, as follows:

$$
\mathcal{C}(I):=\sum_{i=0}^{b-1}\left[\Delta^{i}(I)\right]^{b ! / b-i}
$$

If $Z$ a $B$-inductive hypersurface, then the coefficient ideal relative to $Z$, or the $Z$-coefficient ideal, denoted by $\mathcal{C}(I, Z)$, is the restriction of $\mathcal{C}(I)$ to $Z$. This is a never-zero $Z$-ideal.

The basic object $B_{Z}:=\left(Z, \mathcal{C}(I, Z), b !, E_{Z}\right), E_{Z}=\left(H_{1} \cap Z, \ldots, H_{m} \cap Z\right)$, is called the inductive object of $B$, relative to the inductive hypersurface $Z$.

$(\gamma)$ Consider a $t$-permissible sequence of basic objects and transformations $B_{0} \leftarrow \cdots \leftarrow B_{r}$ (where $\left(B_{j}=\left(W_{j}, I_{j}, b, E_{j}\right)\right)$ and a point $w \in W_{r}$ such that, near $w, M_{w}=\left\{x: t_{r}(x)=t_{r}(w)\right\}$ has codimension (in $\left.W_{r}\right)>1$. Then there is a nice object $B_{r}^{\prime \prime}$, defined on a suitable neighborhood $U$ of $w$ in $W_{r}$, admitting an inductive hypersurface $Z$, such that $\operatorname{Sing}\left(B_{r}^{\prime \prime}\right)=M_{w}$. 
The definition and properties of this object are presented in $[11,9.5]$ or, in a more general context, in Section 8 of this paper.

$(\delta)$ Homogenized ideals and objects. If $W$ is a variety, a $W$-weighted ideal is a pair $(I, b)$, where $I \subset \mathcal{O}_{W}$ is $W$-ideal and $b$ is a non-negative integer.

The associated homogenized ideal of $(I, b)$ is the the $W$-ideal

$$
\mathcal{H}(I, b)=I+\Delta(I) T(I)+\cdots+\Delta^{i}(I) T(I)^{i}+\cdots+\Delta^{b-1}(I) T(I)^{b-1}
$$

where we have written $T(I):=\Delta^{b-1}(I)$ (see [28], Section 2).

If $B=(W, I, b, E)$ is a basic object in $\mathcal{S}$, the basic object $\mathcal{H}(B):=$ $(W, \mathcal{H}(I, b), b, E)$ is the homogenized object associated to $B$. This is discussed in detail (in a more general setting) in Section 7.

2.10. We return to the discussion of the VW-resolution algorithm. In the notation of 2.8, we take as the open set $U$ a neighborhood of $w$ over which the nice object $B_{s}^{\prime \prime}$ of $2.9(\gamma)$ is defined. Then its associated homogenized object $\mathcal{H}\left(B_{s}^{\prime \prime}\right):=\left(H B_{s}^{\prime \prime}\right)$ is again nice and it admits an adapted hypersurface $Z_{s}$ containing $w$, defined on $U$. This will be the $Z_{s}$ of 2.8. Our object $B_{s}^{*}$ of $2.8(\beta)$ will be $\left(\mathcal{H}\left(B_{s}^{\prime \prime}\right)\right)_{Z_{s}}$.

In 8.5 of this paper we shall check that properties (i)-(iv) of $2.8(\beta)$ are valid.

2.11. The algorithm discussed in [11] or [5] is very similar to the VWalgorithm just described. It proceeds as in 2.8 , the only difference is that in the inductive step the auxiliary object $B_{s}^{*}$ now is $\left(B_{s}^{\prime \prime}\right)_{Z_{s}}$ rather than $\left(\mathcal{H}\left(B_{s}^{\prime \prime}\right)\right)_{Z_{s}}$. This looks simpler, however with this approach it is more difficult to check that the process is independent of the choice of the adapted hypersurfaces $Z_{s}$ we choose (as mentioned in the introduction, see also 9.11).

Actually, it can be proved that both the VW-algorithm and that of [11] are the same, in the sense that the resolution functions in either case coincide. We shall not check this fact in this paper.

We want to emphasize that we do not claim that the VW-algorithm (or that of [11]) and that of [28] are the same. They are not, as is proved in [7] Remark 6.16. The VW-algorithm is not that of [28], but essentially that of [11], with some details changed by using some concepts from [28].

2.12. The algorithms just discussed enjoy some important additional properties. For instance, they are functorial with respect to etale morphisms $W^{\prime} \rightarrow W$ (where $W=u s(B), B$ a basic object) and extension of the base field. For the precise statements and a proof see [11] or [5].

2.13. In the following sections ( 3 through 8 ) we attempt to generalize, as much as possible, the theory just described to the case where we do not work over a base field $k$ but rather a (suitable) artinian ring $A$. Our point of view is to regard a basic object defined over $A$ (see 4.1) as an infinitesimal family 
of basic objects, or an infinitesimal deformation of the only (closed) fiber that we have. In our approach, when adapting a given notion is adapted to this situation, the intuitive idea is that it applies to the fiber and it "spreads well" along the (infinitesimal) parameter space $\operatorname{Spec}(A)$. This won't be always possible. When the whole resolution process of the fiber can be extended to an object over $A$ we'll say that we achieved algorithmic equiresolution. The extension of this theory to the mentioned relative situation sometimes is straightforward, sometimes not. In general, when the translation to this relative situation is simple we shall omit the proofs or certain details.

Concerning the algorithm of resolution, working over an artinian ring $A$ the resolution functions $g_{i}$ of 2.6 do not seem very useful. Indeed, these functions are really defined on the underlying topological space $W$ (notation of 2.3), or a subspace thereof. If $W$ is a scheme over $A$ (artinian), $W$ and the fiber share the same topological space. Hence, for our purposes the VWalgorithm should be regarded as a rule selecting the appropriate centers $C_{0}, C_{1}, \ldots$ involved in the algorithmic resolution sequence of 2.6. This is our point of view.

\section{Basic notions}

3.1. In general, we shall use the notation and terminology introduced in 2.1. In addition, throughout, the symbol $\mathcal{A}$ will denote the collection of artinian local rings $(A, M)$ such that the residue field $k=A / M$ has characteristic zero. Since such a ring is necessarily complete and equicharacteristic, $A$ will contain a (unique) field of representatives, i.e. a subfield mapping onto $k$ via the canonical homomorphism $A \rightarrow A / M=k$. Thus, $A$ is automatically a $k$-algebra. If $A \in \mathcal{A}$ we'll usually write $S=\operatorname{Spec}(A)$.

3.2. If $p: W \rightarrow S$ is a smooth morphism, $S=\operatorname{Spec}(A)$, the fiber (or closed fiber) of $p$ is the fiber over the only point of $S$ (regarded as a closed subscheme of $S$ ). It is denoted it by $W^{(0)}$. Then $W^{(0)}=W_{\text {red }}$ and this is an algebraic variety, smooth over the field $A / M$. If $I \subset \mathcal{O}_{W}$ is a $W$-ideal, $I^{(0)}:=I \mathcal{O}_{W^{(0)}}$ is called the fiber of $I$.

A hypersurface on $W$ over $S$, or an $S$-hypersurface, or an $A$-hypersurface, is a positive Cartier divisor $H$, flat over $S$, inducing over the fiber $W^{(0)}$ a regular codimension one subscheme $H^{(0)}$. Thus, the ideal $I(H)_{y}$ (the stalk at $y$ of the ideal sheaf defining $H \subset W)$ is defined by a single element, inducing at $\mathcal{O}_{W^{(0)}, y}$ an element $a$ of order one. This element $a$ is a non-zero divisor of $\mathcal{O}_{W, x}$. This is a consequence of Theorem 23.2 (page 179) in [20] (or use 11.2 , witn $n=1$ ). It can be proved that the naturally induced projection morphism $H \rightarrow S$ is smooth (see 11.2). 
3.3. Let $p: W \rightarrow S$ be as in 3.1, $w$ a point of $W, R=\mathcal{O}_{W, w}, R^{\prime}=\mathcal{O}_{W^{(0)}, w}$ (the local ring of the special fiber at $w$, which is regular.) A system of elements $a_{1}, \ldots, a_{n}$ in $R$ is called a regular system of parameters of $R$ relative to $p$, or simply (if the morphism $p$ is clear) an $A$-regular (or $S$-regular) system of parameters if the induced elements $a_{1}^{(0)}, \ldots, a_{n}^{(0)}$ in $R^{\prime}$ form a regular system of parameters, in the usual sense. Elements $a_{1}, \ldots, a_{r}$ of $R$ are part of an A-regular system of parameters, or a partial A-regular system of parameters, if they are contained in an $A$-regular system of parameters $a_{1}, \ldots, a_{n}$, $r \leq n$, of $R$. Then necessarily $a_{1}, \ldots, a_{n}$ is a regular sequence in the local ring $\mathcal{O}_{W, w}$ (see 11.2, or use the result in [20] cited at the end of 3.2 and induction).

3.4. An collection $E=\left\{H_{1}, \ldots, H_{m}\right\}$ of $S$-hypersurfaces of $W$ is said to have normal crossings if for all points $w \in W$ there is an $A$-regular system of parameters $a_{1}, \ldots, a_{n}$ of $\mathcal{O}_{W, w}$ such that $H_{1} \cup \cdots \cup H_{m}$ is defined at $w$ by a product of elements $a_{i}$, without multiple factors. This product might be empty, hence $=1$ (this happens when no hypersurface $H_{i}$ contains $w$.)

A subscheme $C$ of $W$ (with defining sheaf of ideals $I(C)$ ) is said to have normal crossings with $E$ over $S$ (or relative to $S$ ) if $E$ has normal crossings and for all points $w \in C$, there is an $A$-regular system of parameters $a_{1}, \ldots, a_{n}$ in $\mathcal{O}_{W, w}$ such that the stalk $I(C)_{w}$ is generated by $\left(a_{1}, \ldots, a_{r}\right) \mathcal{O}_{W, w}$ (for some $r \leq n$ ) and for each $H_{i}$ containing $w$, the ideal $I\left(H_{i}\right)_{w}$ is generated by a suitable element $a_{j}, j \in\{1, \ldots, n\}$.

It can be proved that the induced projection $C \rightarrow S$ is smooth, and the blowing-up $W_{1}$ of $W$ with center $C$ is also $S$-smooth (see 11.2).

3.5. An $S$-pair (or $A$-pair) is an ordered pair $(p: W \rightarrow S, E)$, with $p$ smooth and $E=\left(H_{1}, \ldots, H_{m}\right)$ an ordered $m$-tuple of distinct $S$-hypersurfaces on $W$ having normal crossings. We call $W$ the scheme of the pair $(p, E)$. When $A$ is a field which should be clear from the context (e.g., when taking a closed fiber), often we shall write $(W, E)$ for the pair.

A permissible center for the pair $(p, E)$ (as above) is a subscheme $C$ of $W$, having normal crossings with $E$. Then $C$ is automatically smooth over $S(3.4)$.

3.6. We use the notation of 2.1. Let $B=(p: W \rightarrow S, E)$ be a pair over $S, I$ a never zero $W$-ideal and $C \subset W$ be an irreducible $S$-permissible center for the pair $(p, E)$, defined by the $W$-ideal $J \subset \mathcal{O}_{W}$, with generic point $y \in C$.

We shall say that the order of $I$ along $C$ is $\geq m$, written $\nu(I, C) \geq m$, if (in the local ring $\mathcal{O}_{W, y}$ ) we have $I_{y} \subseteq\left(J_{y}\right)^{m}$.

Finally, we write $\nu(I, C)=m$ if $m$ is the largest integer such that $\nu(I, C) \geq m$. 
Example 3.7. Let $W=\mathbf{A}_{A}^{2}=\operatorname{Spec} A[x, y]$ with $A=k[\epsilon]=k[t] /\left(t^{2}\right)$ (and, say, $k=\mathbf{C}), S=\operatorname{Spec} A, W \rightarrow S$ the natural projection, $I=\left(\epsilon x+y^{2}+x^{3}\right)$ $A[x, y], E=\emptyset, C=V(x, y), w$ the "origin", i.e. the maximal ideal $(\epsilon, x, y)$ $A[x, y], b=2$. Then $C$ is $S$-permissible and $I_{w}$ has order 2 , but $\nu(I, C)=1$.

3.8. Let $W \rightarrow S=\operatorname{Spec} A$ (with $A \in \mathcal{A}$ ) be a smooth morphism, $w \in W$, $a_{1}, \ldots a_{r}$ elements of $R:=\mathcal{O}_{W, w}$ which are part of an $A$-regular system of parameters, $J=\left(a_{1}, \ldots, a_{r}\right) R, R^{\prime}=R / J$. Then, the completion $R^{*}$ of $R$ with respect to $J$ is isomorphic to the power series ring in $r$ variables $R^{\prime}\left[\left[x_{1}, \ldots, x_{r}\right]\right]$, where $x_{i}$ corresponds to $a_{i}$, for all $i$ (see 11.6).

Now, with the notation of 3.6, let $w$ be the generic point of the center $C$ and $J=I(C)_{w}$. Then, by definition of permissible center, there is a sequence $a_{1}, \ldots, a_{r}$ which is part of an $A$-regular system of parameters (see 3.3 ) and generates $J$. Then, again with notation of 3.6, $\nu(I, C)=m$ if and only if each $f \in I_{w}$, when regarded as an element of the completion $R^{*}=R^{\prime}\left[\left[x_{1}, \ldots, x_{n}\right]\right]$, $R^{\prime}=\mathcal{O}_{C, w}$, can be written as a power series in $x_{1}, \ldots, x_{r}$ of order $\geq m$, with coefficients in $R^{\prime}$, and for some $f$ that order will be exactly $m$.

3.9. The notions of 3.6 can be described in a more global way as follows (cf. [11] or [5, Section 13]). We retain the assumptions and notation of 3.6. Let $J$ be a $W$-ideal. Define $\Delta(J / S):=J+\mathcal{F}_{d-1}\left(\Omega_{Y / S}\right)$, where $Y:=\mathcal{V}(J)$ and $\mathcal{F}_{d-1}$ denotes the $(d-1)$-Fitting ideal. Then, using well known properties of the objects involved and remarks in 3.8, the following facts are easily verified.

If $w \in C$ is a closed point the stalk $I(C)_{w}$ is generated by an $A$ regular sequence $a_{1}, \ldots, a_{n}$ of $\mathcal{O}_{W, w}$, let $R^{*}=A^{\prime}\left[\left[x_{1}, \ldots, x_{n}\right]\right]$ (with $A^{\prime}=$ $\left.\mathcal{O}_{W, w} /\left(a_{1}, \ldots, a_{n}\right)\right)$ denote the completion of $\mathcal{O}_{W, w}$ with respect to the ideal $\left(a_{1}, \ldots, a_{n}\right)$. Then $\Delta(J / S)_{w} R^{*}$ is the ideal generated by the elements $f \in J_{w}$ and the partials $\partial f / \partial x_{i}, i=1, \ldots, n$, for all $f \in J_{w}$.

We may iterate this construction, getting $W$-ideals $\Delta^{i}(J / S), i=1,2, \ldots$, such that $\Delta^{i}(J / S) R^{*}$ is the ideal generated by elements of $I$ and their partial derivatives of order $\leq i$.

The connection between this object and the notion of 3.6 is given in the following result.

Proposition 3.10. If $(W \rightarrow S, E)$ is an $S$-pair, $C$ is an irreducible permissible center, with generic point $y$ and defining ideal $I(C)=J$, and $I$ a never-zero $W$-ideal, then the following statements are equivalent:

(i) $I_{y} \subseteq\left(J_{y}\right)^{b}$ (i.e., $\nu(I, C) \geq b$ ).

(ii) $I_{x} \subseteq\left(J_{x}\right)^{b}$ for every closed point $x$ in a dense open subset of $C$ (i.e., a non-empty subset of $C)$.

(iii) $\Delta^{b-1}(I / S)_{x} \subseteq J_{x}$ for every closed point $x$ in a dense open subset of $C$ (say, for $x$ in $U \cap C, U$ a suitable open set of $W$.)

(iv) $\Delta^{b-1}(I / S) \subseteq J$ 
Proof. The other implications being well-known, we discuss the implication $(i i i) \Rightarrow(i v)$. Recall the following basic facts. Here, if $Y$ is a locally closed subscheme of a scheme $W, \operatorname{cl}(Y)$ denotes the scheme-theoretic closure of $Y$ in $W$.

(a) If $Y$ is a closed subscheme of $W, U$ an open of $W, Y^{\prime}=Y \cap U$ then $\operatorname{cl}\left(Y^{\prime}\right) \subseteq Y$.

(b) In the notation of (a) if, moreover, the closed subscheme $Y$ is irreducible, has no embedded points (e.g., if it is C-M, that is a Cohen-Macaulay scheme) and $U$ and $Y$ have at least one point in common, then $\operatorname{cl}\left(Y^{\prime}\right)=Y$

Now we prove $(i i i) \Rightarrow(i v)$ as follows. Let $Y=\mathcal{V}\left(\Delta^{b-1}(I / S)\right)$, note that $\mathcal{V}(I(C))=C$. Observe, moreover, that $W$ is C-M (use the fact that $W$ is smooth over $S$ which is C-M, now use the Corollary to Thm. 23.3, page 181 of [20]). Since $C$ is locally defined by a regular sequence, $C$ is also C-M, in particular with no embedded points. Let $U$ be an open set in $W$ such that the open subset of $C$ in (iii) is of the form $C^{\prime}=C \cap U$. Let $Y^{\prime}=Y \cap U$, and $C^{\prime}=C \cap U$. The assumption of $($ iii) implies that the restriction of $\Delta^{b-1}(I / S)$ to $U$ is contained in the restriction of $I(C)$ to $U$ (because the closed points are dense, $W_{\text {red }}$ being an algebraic scheme over a field). This implies, taking $\mathcal{V}$, that $C^{\prime} \subseteq Y^{\prime}$. Taking closure we get $\operatorname{cl}\left(C^{\prime}\right) \subseteq \operatorname{cl}\left(Y^{\prime}\right)$. But, by (a) and (b), $c l\left(C^{\prime}\right)=C$ and $c l\left(Y^{\prime}\right) \subseteq Y$. Thus, $C \subseteq Y$, which is equivalent to $(i v)$.

3.11. Note that the notion of $S$-hypersurface given in 3.2 may be equivalently expressed as follows. A subscheme $H$ of $W$ is an $S$-hypersurface if, for each $x \in H, I(H)_{x}$ is generated by an element $a \in \mathcal{O}_{W, x}$ whose image in $\mathcal{O}_{W^{(0)}, x}$ is in $M \backslash M^{2}$ (with $M=r\left(\mathcal{O}_{W^{(0)}, x}\right)$ ). Indeed, as remarked in 3.2, the definition given there implies this notion. Conversely, if $H \subset W$ is as indicated, then $H$ is flat over $S$ (see the end of 3.4) and, using 3.8, the completion of $R=\mathcal{O}_{W, x}$ with respect to $(a)$ is isomorphic to $A^{\prime}[[a]]$ (with $a$ analytically independent over $\left.A^{\prime} \cong A /(a)\right)$, showing that $a$ is not a zero divisor in $\mathcal{O}_{W, x}$. Hence, $H$ is a relative Cartier divisor over $S$.

3.12. Let $(p: W \rightarrow S, E)$ be an $S$-pair, $C$ an $S$-permissible center of it. If $W^{\prime}$ is the blowing up of $W$ with center $C, p^{\prime}: W^{\prime} \rightarrow S$ the induced morphism and $E^{\prime}=\left(H_{1}^{\prime}, \ldots, H_{m}^{\prime}, H_{m+1}^{\prime}\right)$, where $H_{i}^{\prime}$ is the strict transform of $H_{i}$, for $i=1, \ldots, m$ and $H_{m+1}^{\prime}$ is the exceptional divisor, then $\left(p^{\prime}, E^{\prime}\right)$ is a new $S$-pair, called the transform of $(p, E)$ with center $C$. The fact that $H_{m+1}^{\prime}$ is an $A$-hypersurface (in the sense of 3.2 ) is seen by using 3.11 , the other points are easy. The closed fiber of $\left(p^{\prime}, E^{\prime}\right)$ may be identified to the transform of $\left(W^{(0)}, E^{(0)}\right)$ with center $C^{(0)}:=C \cap W^{(0)}$ (use 11.7). 


\section{Basic objects}

Definition 4.1. A basic object over $S$ is a four-tuple $B=(p: W \rightarrow$ $S, I, b, E)$, where $(p, E)$ is an $S$-pair, $I$ a never-zero $W$-ideal (2.1) and $b$ is a non-negative integer.

The pair $(p, E)$ is the underlying pair of the basic object $B$ and $W$ is the underlying scheme of the basic object $B$, denoted by us $(B)$. The integer $b$ is the index of the $A$-basic object $B$. The dimension of $B$, $\operatorname{dim}(B)$, is the dimension of the scheme $u s(B)$.

There is a naturally defined notion of the fiber of a basic object $B$, usually denoted by $B^{(0)}=\left(W^{(0)}, I^{(0)}, b, E^{(0)}\right)$. We let $\operatorname{Sing}\left(B^{(0)}\right)$ denote the closed set $\left\{w \in W^{(0)}: \nu_{w}\left(I^{(0)}\right) \geq b\right\}$ and $\operatorname{Sing}(B):=\operatorname{Sing}\left(B^{(0)}\right)$.

4.2. If $B=(p: W \rightarrow S, I, b, E)$ is an $S$-basic object, a subscheme $C \subseteq W$ is a permissible center for $B$ (or $B$-permissible, or just a $B$-center) if it is a permissible center for its underlying $S$-pair $(p, E)$ and moreover, for each irreducible component $C^{\prime}$ of $C$ we have, letting $C^{\prime(0)}$ be the fiber of the natural projection $C^{\prime} \rightarrow S$, that

$$
\text { (1) } \nu\left(I, C^{\prime}\right)=\nu\left(I^{(0)}, C^{\prime(0)}\right) \geq b
$$

with the notation of 3.6.

Note that if $A=k$ (a field) then a $B$-permissible center is a permissible center in the sense of [11], [5] or 2.5. A $B$-permissible center induces a permissible center on the fiber $B^{(0)}$.

Example 4.3. It is possible to have $A$-basic objects without any permissible centers. This is a simple example.

Let $B=(W \rightarrow S, I, 2, \emptyset)$, where $S=\operatorname{Spec}(A)$, with $A=k[\epsilon](k$ a characteristic zero field, $\left.\epsilon^{2}=0,\right), W=\operatorname{Spec}(A[x])$ ( $x$ an indeterminate) $I=\left(x^{2}, \epsilon x\right)$. Were $C$ a $B$-permissible center, it should induce the only permissible center of the fiber $B^{(0)}$, namely the origin $\mathcal{V}(x)$. Hence, the ideal $I(C)$ is generated by an element of the form $y=x+h, h \in(\epsilon) A[x]$ and $A[x]=A[y]$. Then $I=\left((y-h)^{2}, \epsilon(y-h)\right)=\left(y^{2}, \epsilon y\right)$ and $C=\mathcal{V}(y)$. Now it is clear that $\nu(I, C)=1$ while $\nu\left(I^{(0)}, C^{(0)}\right)=2$, impossible were $C$ a $B$-permissible center.

Proposition 4.4. (a) Let $C$ be an irreducible B-center, with $B$ as in 4.1, $Z \subset W$ an irreducible $S$-hypersurface, both $C$ and $Z$ having a common point $y$. Assume $I(Z)_{y} \subseteq I(C)_{y}$. Then, $C \subset Z$.

(b) Assume $C, C^{\prime}$ are irreducible B-centers, having a common point $y$, and $I(C)_{y}=I\left(C^{\prime}\right)_{y}$. Then $C=C^{\prime}$. 
Proof. It is similar to that of 3.10. (a) Let $c l$ indicate scheme-theoretic closure in $W$. The hypothesis implies that there is an open dense neighborhood $U$ of $y$ in $W$ such that, letting $C_{1}:=C_{\mid U}, Z_{1}:=Z_{\mid U}$, we get $C_{1} \subseteq Z_{1}$. Hence $\operatorname{cl}\left(C_{1}\right) \subseteq \operatorname{cl}\left(Z_{1}\right)$. But since both $C$ and $Z$ are Cohen-Macaulay, hence without non-trivial embedded points, necessarily $\operatorname{cl}\left(C_{1}\right)=C, \operatorname{cl}\left(Z_{1}\right)=Z$.

(b) Use the same argument as in (a), with $C^{\prime}$ rather than $Z$, to get $C \subseteq C^{\prime}$. Similarly we get $C^{\prime} \subseteq C$.

4.5. If $B$ is an $A$-basic object, $C$ an $A$-permissible center for $B$ and $\pi$ : $W_{1} \rightarrow W$ the blowing-up of $W$ with center $C$, the sheaf $I$ induces several important sheaves of $\mathcal{O}_{W_{1}}$-ideals. Namely, we have:

(i) $I_{1}^{\prime}=I \mathcal{O}_{W_{1}}$ (the total transform of $I$ to $W_{1}$ ),

(ii) $I_{1}:=\mathcal{E}^{-b} I_{1}^{\prime}$, where $\mathcal{E}$ defines the exceptional divisor (the controlled transform of $I$ to $W_{1}$ ),

(iii) $\overline{I_{1}}:=\mathcal{E}^{-a} I^{\prime}{ }_{1}$, with $a$ as large as possible (the proper transform of $I$.) (If $C$ is not connected, the exponent $a$ is constant along $p^{-1}\left(C^{\prime}\right)$, for each connected component $C^{\prime}$ of $C$, but not necessarily globally constant).

4.6. Given a $A$-basic object $B$ (as in 4.2), the 4 -tuple $B_{1}:=\left(W_{1} \rightarrow\right.$ $S, I_{1}, b, E_{1}$ ) (where $I_{1}$ is the controlled transform of $I$ and $E_{1}$ is defined as in 3.5) is a new $A$-basic object, called the transform of the basic object $B$ with center $C$ (or at $C$ ). The process of replacing a basic object $B$ by its transform $B_{1}$ (with a permissible center $C$, as above) will be called the transformation of $B$ with center $C$, indicated by $B \leftarrow B_{1}$, if $C$ is clear. Sometimes we shall write $\mathcal{T}(B, C)$ to denote the transform of $B$ with center $C$.

Using the statement at the end of 3.12, one may verify that the transform of an $S$-basic object with a $B$-permissible center over $S$ induces (by taking fibers) the transform of the fiber $B^{(0)}$ with center $C^{(0)}:=C \cap W^{(0)}$ (notation as in 3.12). Moreover, ${\overline{I_{1}}}^{(0)}=\overline{I^{(0)}{ }_{1}}$.

When $A=k=$ a field, the notions of 4.5 and 4.6 reduce themselves to those of 2.5 .

4.7. A permissible sequence of transformations over $S$ is one of the form:

$$
\text { (1) } \quad B_{0} \leftarrow \cdots \leftarrow B_{r}
$$

where each arrow $B_{i} \leftarrow B_{i+1}$ stands for a transformation of basic objects, with a $B_{i}$-permissible center, say $C_{i} \subset W_{i}=u s\left(B_{i}\right)$. Note that, by taking fibers, such a sequence induces one:

$$
B_{0}^{(0)} \leftarrow \cdots \leftarrow B_{r}^{(0)}
$$

where the $i$-th arrow is the transformation with permissible center $C_{i-1}^{(0)} \subset$ $u s\left(B_{i}^{(0)}\right)$, where $C_{i-1}^{(0)}$ is the restriction of $C_{i-1}$ to the fiber. The sequence 
obtained from (1) by deleting $B_{j+1}, \ldots, B_{r}$ and the corresponding arrows is called the $j$-truncation of the sequence (1).

The sequence (1) is called an equiresolution of the basic object $B_{0}$ if $\operatorname{Sing}\left(B_{r}\right)=\emptyset$

4.8. $W$-equivalence. Let $B=(W \rightarrow S, I, b, E)$ and $B^{\prime}=\left(W \rightarrow S, J, c, E^{\prime}\right)$ be basic objects $(S=\operatorname{Spec}(A), A$ as in 3.1) be basic objects over $S$. We shall say that they are pre-equivalent if the following conditions hold: (0) $C \subset W$ is a $B$-permissible center if and only if it is a $B^{\prime}$-permissible center, (1) If $B_{1}$ (resp. $B_{1}^{\prime}$ ) denotes the transform of $B$ (resp. $B^{\prime}$ ) with center $C$, then $C_{1}$ is a $B_{1}$-permissible center if and only if it is a $B_{1}^{\prime}$ permissible center, consider the transforms with center $C_{1}, \ldots,(n)$ if we repeat this process $n$ times $(n$ any natural number), obtaining basic objects $B_{n}$ and $B_{n}^{\prime}$ respectively, $C_{n}$ is a $B_{n}$-permissible center if and only if it is a $B_{n}^{\prime}$-permissible center.

We say that $B$ and $B^{\prime}$ are $W$-equivalent if, in addition, the special fibers $B^{(0)}$ and $B^{\prime(0)}$ are also pre-equivalent.

In case where the base is a field, pre-equivalence is the same as equivalence. This notion is what in [28] is called equivalence. The notion of equivalence used in [11] requires a further condition, for that reason we use our terminology of $\mathrm{W}$-equivalence.

Example 4.9. Let $B=(W \rightarrow S, I, b, 2, \emptyset)$ and $B=\left(W \rightarrow S, I^{\prime}, b, 2, \emptyset\right)$, where $S=\operatorname{Spec} A), A=k[\epsilon], k$ a field, $\epsilon^{2}=0, W=\operatorname{Spec}(A[x]), I=$ $\left(x^{2}+\epsilon x\right), I^{\prime}=\left(x^{5}, \epsilon x\right)$. Then, the $A$-basic objects $B$ and $B^{\prime}$ are preequivalent but not equivalent.

\section{Some resolution tools}

In this section we study, in the context of $A$-basic objects $(A \in \mathcal{A})$, some notions discussed in 2.7 (where we worked over a field).

5.1. $\omega$ - and t-permissible centers. Consider a permissible sequence of $A$ basic objects

$$
\text { (1) } B_{0} \leftarrow B_{1} \leftarrow \cdots \leftarrow B_{r}
$$

where $B_{i+1}=\mathcal{T}\left(B_{i}\right), C_{i}$ a $B_{i}$-permissible center, for all $i$. We want to define, recursively on the length $r$, the notion "(1) is an $\omega$-permissible sequence, or an $\omega$-sequence" (see 2.7, where $A=k$, a field). If $r=0$, by definition (1) is always $\omega$-permissible. Assume we know, by induction, what is an $\omega$ permissible sequence when the length is $r$, and that this implies that the induced sequence of special fibers

$$
B_{0}^{(0)} \leftarrow B_{1}^{(0)} \leftarrow \cdots \leftarrow B_{r}^{(0)}
$$


is such that each center $C_{i}^{(0)}$ that was used satisfies $C_{i}^{(0)} \subseteq \operatorname{Max}\left(\omega_{i}\right)$ (i.e., is $\omega$-permissible). Given a sequence of length $r+1$, we say it is $\omega$-permissible if its $r$-truncation is so (then we have a function $\omega_{r}$ with $\operatorname{domain} \operatorname{Sing}\left(B_{r}^{(0)}\right):=$ $\operatorname{Sing}\left(B_{r}\right)$. Let $\left.b_{r} / b:=\max \left(\omega_{r}\right)\right)$ and if the center $C_{r}$ used to obtain $B_{r+1}$ satisfies $\nu\left(\overline{I_{r}}, C_{r}\right)=\nu\left(\overline{I_{r}^{(0)}}, C_{r}^{(0)}\right)=b_{r}$ (where, e.g., $\overline{I_{r}}$ demotes proper transform, see 4.5). Then the induced sequence of fibers satisfies $C_{i}^{(0)} \subseteq \operatorname{Max}\left(\omega_{i}\right)$ for all $i$, with notation as above. Given a $\omega$-permissible sequence (1), each center $C_{j}$ used in it is said to be $\omega$-permissible for $B_{j}$. If (1) is an $\omega$ permissible sequence, by the function $\omega_{j}$ of (1) we mean the function $\omega_{j}$ of the corresponding sequence of fibers.

Now we study $t$-permissible sequences. Consider a sequence of $A$-basic objects and transformations (1), where we write $B_{j}=\left(W_{j} \rightarrow S, I_{j}, b, E\right)$, for all $j$. We shall define, by induction on the length $r$, what it means that (1) is $t$-permissible.

If $r=0$ (i.e. there is just one basic object), the sequence (reduced to one object) is $t$-permissible.

Next assume the notion of $t$-permissible center is defined, by induction, if the sequence has length $\leq r$, in such a way that it induces a sequence of fibers which is $t$-permissible, in the sense of 2.8. We declare a sequence of length $r+1 t$-permissible if the following conditions (a) and (b) hold: (a) the $r$-truncation of (1) is $t$-permissible . Hence by looking at fibers we have functions $t_{i}, i=1, \ldots, r$ satisfying $\max \left(t_{i}\right) \geq \max \left(t_{i+1}\right), i=0, \ldots, r$. Let $s$ be the smallest index such that $\max \left(\omega_{s}\right)=\max \left(\omega_{r}\right)$. Let $E_{r}^{-}$consist of the hypersurfaces in $E_{r}$ which are strict transforms of those in $E_{s}$ and $(\bar{\omega}, \bar{n})=\max \left(t_{r}\right)$. Then we demand: (b) any component $C$ of the center $C_{r}$ used to obtain $B_{r+1}$ satisfies: $\nu\left(\overline{I_{r}}, C\right)=\nu\left(\overline{I_{r}^{(0)}}, C^{(0)}\right)=b_{r}$ and for each closed point $y \in C$, the number of hypersurfaces in $E_{r}^{-}$containing $y$ is as equal to $\bar{n}$. If (1) is a $t$-permissible sequence, each center $C_{j}$ used in it is said to be $t$-permissible for $B_{j}$, and by the function $t_{j}$ of (1) we mean the function $t_{j}$ of the corresponding sequence of fibers.

5.2. We shall consider, in the present situation, the analogue of certain numerical invariants introduced in [26] (see also [27], where it is proved that the algorithm is equivariant, or [11], which is an exposition of these two papers). But we shall use, to simplify, a different notation. Consider a permissible sequence of $A$-basic objects and transformations as in (1) of 5.1, which is also $t$-permissible.

If $C$ is any irreducible permissible center for $B_{j}, j=0, \ldots r$, we define

$$
\omega_{j}(C):=\nu\left(\bar{I}_{j}, C\right) / b, \quad \sigma_{j}(C):=\nu\left(I_{j}, C\right) / b
$$


where we have used the notation of 3.6 and 4.5. In [11] these numbers are denoted by w-ord and ord respectively.

Proposition 5.3. With the notation and assumptions introduced in 5.2, let $C$ be an irreducible permissible center for $B_{r} D_{i}^{\prime}$ its image by the natural morphism $W_{r} \rightarrow W_{i}:=u s\left(B_{i}\right)$, assume $D_{i}^{\prime} \subseteq C_{i}$ and let $D_{i}$ be the irreducible component of the center $C_{i}$ containing $D_{i}^{\prime}, i=0, \ldots, r-1$. Then,

$$
\omega_{r}(C)=\sigma_{r}(C)-\sigma_{r-1}\left(D_{r-1}\right)-\cdots-\sigma_{0}\left(D_{0}\right)+r
$$

Proof. Note that if $B_{j}=\left(W_{j} \rightarrow S, I_{j}, b, E_{j}\right)$, with $E_{j}=\left(H_{1}, \ldots, H_{m+j}\right)$, where $H_{1}, \ldots, H_{m}$ are the strict transforms of the hypersurfaces in $E_{0}$, then we have an expression

$$
\text { (1) } \quad I_{j}=I\left(H_{m+1}\right)^{a_{1}} \ldots I\left(H_{m+j}\right)^{a_{j}} \overline{I_{j}}
$$

where each exponent $a_{i}$ is constant on each irreducible component of $H_{i}$. From this (for $j=r$ ), taking orders along $C$ (see 3.6) we get:

$$
\nu\left(I_{r}, C\right)=\nu\left(\overline{I_{j}}, C\right)+\sum_{j=1}^{r} a_{j}(y)
$$

where $y$ is the generic oint of $C$. Note that if $y_{j}$ is the image of $y$ in $W_{j}$ via the appropriate morphism,

$$
a_{j}\left(y_{j}\right)=\nu\left(I_{j-1}, D_{j-1}\right)-b
$$

From this, iterating, we obtain:

$$
\nu\left(\overline{I_{r}}, C\right)=\nu\left(I_{r}, C\right)-\left(\sum_{i=0}^{r-1} \nu\left(I_{i}, D_{i}\right)\right)+r b
$$

Dividing by $b$ we obtain the desired formula.

We shall discuss the notion of monomial objects $(2.7(i i)$ or [11], Section 5$)$ in the context of basic objects over a ring $A \in \mathcal{A}$.

5.4. Monomial objects. Using the notation of $2.7(i i)$, let $B=(W, I, b, E)$ (where $E=\left(H_{1}, \ldots, H_{m}\right)$ ) be a monomial object (with $W$ a $k$-variety, $k$ a characteristic zero field). We define functions $\Gamma_{i}, i=1,2,3$, with domain $S:=\operatorname{Sing}(B)$, as follows.

If $w \in S, \Gamma_{1}(w)$ is the smallest integer $p$ such that there are indices $i_{1}, \ldots, i_{p}$ such that

$$
\alpha_{i_{1}}(w)+\cdots+\alpha_{i_{p}}(w) \geq b
$$


Consider, for $w \in S$, the set $P^{\prime}(w)$ of sequences $i_{1}, \ldots, i_{p}$ satisfying (1) above, and let $\Gamma_{2}(w)$ be the maximum of the rational numbers $\left(\alpha_{i_{1}}(w)+\right.$ $\left.\cdots+\alpha_{i_{p}}(w)\right) / b$, for $\left(i_{1}, \ldots, i_{p}\right) \in P^{\prime}(w)$.

If $w \in S$, let $P(w)$ be the set of all sequences $\left(i_{1}, \ldots, i_{p}, 0,0, \ldots\right)$ such that $\left(\alpha_{i_{1}}(w)+\cdots+\alpha_{i_{p}}(w)\right) / b=\Gamma_{2}(w)$, and define $\Gamma_{3}(w)$ to be the maximum of the set $P(w)$, when we use the lexicographical order.

Finally, one defines a function $\Gamma$ (or $\Gamma_{B}$ ) from $S$ to $\mathbf{Z} \times \mathbf{Q} \times \mathbf{Z}^{\mathbf{N}}$ by the formula $\Gamma(w)=\left(-\Gamma_{1}(w), \Gamma_{2}(w), \Gamma_{3}(w)\right)$. When the target is lexicographically ordered the function $\Gamma$ is upper semicontinuous.

Let $\max \left(\Gamma_{3}\right)=\left(i_{1}, \ldots, i_{p}, 0,0, \ldots\right)$ and take $C=H_{i_{1}} \cap \cdots \cap H_{i_{p}}$. Then, it turns out that $C$ is a permissible center for the pair $(W, E)$ and that the transform $B_{1}$ of $B$ is again monomial, satisfying $\max \left(\Gamma_{B_{1}}\right)<\max \left(\Gamma_{B}\right)$. Thus, iterating this process, after a finite number of steps we reach a situation where the singular locus is empty. (See [11, Section 5] or [5, Section 20]).

Now consider $A$-basic objects $B=(W \rightarrow S, I, b, E), E=\left(H_{1}, \ldots, H_{m}\right)$ $S=\operatorname{Spec}(A) A \in \mathcal{A}$. We shall say that such an object is premonomial if its closed fiber $B^{(0)}$ is monomial. Let $\Gamma:=\Gamma_{B^{(0)}}$. We say that our $A$-basic object $B$ is monomial if it is premonomial and, letting $\left(i_{1}, \ldots, i_{p}, 0,0, \ldots\right)=$ $\max \left(\Gamma_{3}\right)$, then $C:=H_{i_{1}} \cap \cdots \cap H_{i_{p}}$ is a $B$-permissible center. This is called the canonical center of the monomial $A$-basic object $B$.

The following proposition is an easy consequence of the definitions.

Proposition 5.5. Let $B$ be a monomial A-basic object $B, C:=H_{i_{1}} \cap \cdots \cap H_{i_{p}}$ its canonical center. Let $H_{i}$ induce $H_{i}^{(0)}$ on the special fiber $B^{(0)}$. Then, if $B_{1}$ is the transform of $B$ with center $C$, the closed fiber $B^{(0)}$ is naturally isomorphic to the transform of $B^{(0)}$ with center $H_{i_{1}}^{(0)} \cap \cdots \cap H_{i_{p}}^{(0)}$, and this is the canonical center of $B^{(0)}$.

It immediately follows from the proposition that if $B$ is monomial, with canonical center $C$, then the transform $B_{1}$ of $B$ at $C$ is again premonomial.

Definition 5.6. If $B$ is a basic object over $A$, we say that $B$ admits a $\Gamma$ permissible center if: (a) $B$ is monomial, (b) the transform $B_{1}$ of $B$ at the canonical center is again monomial.

5.7. $\rho$-permissible sequences. A sequence $B_{0} \leftarrow \cdots \leftarrow B_{r}$ of $A$-basic objects and transformations is called $\rho$-permissible if there is an integer $s \geq 0$ such that: (a) $B_{0} \leftarrow \cdots \leftarrow B_{s}$ is $t$-permissible, (b) $B_{j}$ is monomial if $s \leq j$ and, for all such $j, B_{j} \leftarrow B_{j+1}$ is the transformation with the canonical center of $B_{j}$. In particular, it could be $s>r$, in this case the sequence is $t$-permissible, or $s=0$, in this case all the objects are monomial and the transformations have canonical centers. 


\section{The inductive object}

6.1. Throughout this section, $A$ denotes an artinian ring in $\mathcal{A}(3.1)$ and $S=$ $\operatorname{Spec}(A)$. Given an $A$-basic object $B=(W \rightarrow S, I, b, E), E=\left(H_{1}, \ldots, H_{m}\right)$, we say that an $S$-hypersurface $Z \subset W$ is transversal to $E$ if for each closed point $w$ in $Z$, there is an $A$-regular system of parameters $a_{1}, \ldots, a_{n}$ of $\mathcal{O}_{W, w}$ such that $I(Z)_{w}$ is defined by $a_{1}$ and for every $H$ in $E$ containing $w$, the ideal $I(H)_{w}$ is defined by some $a_{i}$, with $i>1$. In particular, $Z$ has normal crossings with $E$, in the sense of 3.4 .

6.2. Adapted and inductive hypersurfaces, nice objects. Given an A-basic object $B=(W \rightarrow S, I, b, E)$ as above, we say that an $S$-hypersurface $Z \subset W$ is adapted to $B$ (or that $Z$ is $B$-adapted) if the following conditions hold:

$(\mathrm{A} 1) \quad I(Z) \subseteq \Delta^{b-1}(I / S)$ (an inclusion of sheaves of $\mathcal{O}_{W}$-ideals),

(A2) $Z$ is transversal to $E$.

If $Z$ is adapted and, moreover, it satisfies

(A3) Whenever $D$ (resp. $D^{\prime}$ ) is an irreducible component of $Z$ (resp. of $\left.V\left(\Delta^{b-1}(I / S)\right)\right)$ we have $D \neq D^{\prime}$, then we say that $Z$ is $B$-inductive (or just inductive, if $B$ is clear).

In [11] the analogues of (A1) and (A2) are called (LC) and (IA), respectively. Condition (A3) does not follow from the others. For instance, if $B$ is the basic object (Spec $(\mathbf{C}[x, y],(y), 1, \emptyset)$ and $Z$ is the line $V(y)$, then $Z$ satisfies (A1) and (A2) but $D=Z$ is a component of both $Z$ and $V\left(\Delta^{b-1}(I / S)\right)$. But if $\operatorname{Sing}(B)$ has codimension at least two, then any $B$-adapted hypersurface is automatically inductive.

We shall say that $B$ (a basic object over $A$ ) is nice if either $\operatorname{Sing}(B)$ is empty or $B$ admits an adapted hypersurface. We say that $B$ is nice at $w \in W$ if there is an open neighborhood $U$ of $w$ such that the restriction of $B$ to $U$ is nice; $B$ is locally nice if $B$ is nice at $w$, for any $w \in W$.

6.3. Remarks on hypersurfaces. We keep the previous assumptions and notation. Recall that, according to 3.2 and 3.11, if $Z \subset W$ is a regular $A$ hypersurface and $w \in Z$ is a closed point, then the stalk $I(Z)_{w}$ is generated by an element $a \in \mathcal{O}_{W, w}$ which is part of an $A$-regular system of parameters 3.3. In particular, if $a_{1}, \ldots, a_{d}$ form an $A$-regular system of parameters of $\mathcal{O}_{W, w}, J:=\left(a_{1}, \ldots, a_{d}\right)$ and $a \in J$, then the order of the generator $a$ with respect to $J$ is $=1$.

Now assume that the regular hypersurface $Z$ also satisfies (A1), i.e., $I(Z) \subseteq \Delta^{(b-1)}:=\Delta^{b-1}(I / S)$. Then, if $w \in Z$, the generator $a \in I(Z)_{w}$ 
defining $Z$ near $w$ belongs to the stalk $\left[\Delta^{(b-1)}\right]_{w}$. If $C$ is a $B$-permissible center, then by (A1) and 3.10 necessarily $C \subseteq Z$. If $w \in C$, then $J:=$ $I(C)_{w}$ is generated by part of an $A$-regular system of parameters. Since $C \subseteq Z, a \in J$ and hence the order of $a$ with respect to $J$ is 1 . Thus we see that $\left[\Delta^{(b-1)}\right]_{w}$ contains an element which is part of an $A$-regular system of parameters.

Conversely, if $w \in W$ and $\left[\Delta^{(b-1)}\right]_{w}$ contains an element $a$ which is part of an $A$-regular system of parameters, then $a$ defines, near $w$, an $A$-regular hypersurface satisfying condition (A1).

Lemma 6.4. If $B$ is nice basic object over $A$, then its fiber $B^{(0)}$ is also a nice basic object (over the residue field of $A$ ).

The proof is simple and will be omitted.

6.5. If $B$ is a nice object and $C \subset W$ is an irreducible $B$-permissible center, with generic point $y$, then $\nu(C, I)=b$. To see this, it suffices to show that if $w$ is a closed point of $\operatorname{Sing} B$, then the stalk $\left[\Delta^{(b-1)}\right]_{w}$ contains contains an element which is part of an $A$-regular system of parameters. But if $Z$ is any adapted hypersurface, from condition (A1) and the inclusion $\Delta^{b-1}(I / S) \subseteq$ $I(C)$ we obtain $I(Z) \subseteq I(C)$. From this, our assertion follows from 6.3 , taking $J=I(C)_{y}$.

6.6. Recall that if $B=(W, I, b, E)$ is a basic object over a field, then it is called good if $\nu_{w}(I)=b$ for any $w \in \operatorname{Sing}(B)$ (see [11]). Working over $A$ artinian, we shall say that an $A$-basic object $B$ is good if its special fiber $B^{(0)}$ is good, in the sense just mentioned. The result of 6.5 says that, working over a field, a nice object is good. Since the fiber of a nice $A$-object is a nice $k$-object ( $k$ being the residue field of $A$ ), the same is true working with $A$-basic objects.

Remark 6.7. Given a good $A$-basic object $B=(W \rightarrow S, I, b, E)$, a closed subscheme $C$ of $W$ which is a center for the pair $(W \rightarrow S, E)$ (see 3.5) is also a $B$-permissible center if and only if $\Delta^{b-1}(I / S) \subseteq I(C)$. In other words, the extra condition $\nu\left(I, C^{\prime}\right)=\nu\left(I^{(0)}, C^{\prime(0)}\right)$, for any component $C^{\prime}$ of $C$, automatically follows. Indeed, one implication is clear, for the other note that from the inclusion and the assumption that $B$ is good we get $b \leq \nu\left(I, C^{\prime}\right) \leq \nu\left(I^{(0)}, C^{\prime(0)}\right)=b$, hence all of these are equalities.

6.8. The notion of adapted hypersurface is stable under permissible transformation, in the sense that if $B \leftarrow B_{1}=\left(W_{1} \rightarrow S, I_{1}, b, E_{1}\right)$ is a transformation along a $B$-permissible center $C$ and $Z_{1}$ is the strict transform of $Z$ to $W_{1}$, then $Z_{1}$ is an adapted hypersurface for $B_{1}$. The stability of condition (A1) follows from Lemma 6.10 below (Giraud's Lemma), part (b), 
and that of (A2) is well known. Lemma 6.10 follows from Lemma 6.9, to be given next. The proofs of both lemmas are entirely analogous to those given in 9.1 and 9.2 of [11], and will be omitted. Indeed, thanks to the remarks in 3.8, the necessary calculations in completions of rings (discussed in $[11$, Section 9]) can be carried out in our case.

Lemma 6.9. Using the notation of 6.8 , let $B \leftarrow B_{1}$ be a permissible transformation of $A$-basic objects, $H \subset W_{1}$ the exceptional divisor, with defining ideal $I(H)$. Then,

(i) $\Delta^{b-i}(I / S) \mathcal{O}_{W_{1}} \subseteq I(H)^{i}$,

(ii) $I(H)^{-i} \Delta^{b-i}(I / S) \mathcal{O}_{W_{1}} \subseteq \Delta^{b-i}\left(I_{1} / S\right)$.

Lemma 6.10. Consider an A-basic object $B=(W \rightarrow S, J, b, E)$, an $A$ adapted hypersurface $Z \subset W$, an irreducible $B$-permissible center $C \subset Z$ and $B_{1}=\left(W_{1} \rightarrow S, J_{1}, b, E_{1}\right)$, the transform of $B$ with center $C$. Let $B^{\prime}=$ $(W \rightarrow S, I(Z), 1, E)$. Then :

(a) $C$ is a $B^{\prime}$-permissible center, and if $B_{1}^{\prime}=\left(W_{1} \rightarrow S, I(Z)_{1}, 1, E_{1}\right)$ is the the transform of $B^{\prime}$ with center $C$, then $I(Z)_{1}=I\left(Z_{1}\right)$, where $Z_{1}$ is the strict transform of $Z$ to $W_{1}$,

(b) $I\left(Z_{1}\right) \subset \Delta^{b-1}\left(J_{1} / S\right)$.

6.11. The coefficient ideal and the object $B_{Z}$. We use the notation of 6.1. Let $B$ be a nice $A$-basic object and $Z$ a $B$-inductive hypersurface.

(a) We define a sheaf of $\mathcal{O}_{W}$-ideals, called the coefficient ideal and denoted by $\mathrm{e}(I / S)$, as follows:

$$
\mathcal{C}(I / S):=\sum_{i=0}^{b-1}\left[\Delta^{i}(I / S)\right]^{b ! / b-i}
$$

Often, when $A$ is a field we'll simply write $\mathrm{C}(I)$. If $B$ is nice and $Z$ a $B$ inductive hypersurface, we define a sheaf of ideals on $Z$, called the coefficient ideal relative to $Z$, or the $Z$-coefficient ideal, denoted by $\mathcal{C}(I / S, Z)$, as the restriction of $\mathrm{e}(I / S)$ to $Z$.

(b) The $A$-basic object $B_{Z}:=\left(Z \rightarrow S, \mathrm{C}(I / S, Z), b\right.$ !, $\left.E_{Z}\right)$, where we write $E_{Z}:=\left(H_{1} \cap Z, \ldots, H_{m} \cap Z\right)$ is called the inductive object of $B$, relative to the inductive hypersurface $Z$. Indeed, this is an $A$-basic object. The fact that $E_{Z}$ is a system of $A$-hypersurfaces with normal crossings follows from (A2); what is left is to check that $\mathrm{e}(I / S, Z)$ is a never zero sheaf of ideals, and this follows from (A3). 
6.12. Condition (A3) is important. For instance, if the basic object is $B=$ (Spec $\left(\mathbf{C}[x, y],\left(y^{2}\right), 2, \emptyset\right)$ and $Z$ is the line $V(y)$, then $\mathcal{C}\left(\left(y^{2}\right)\right)$ restricts the zero sheaf of ideals on $Z$. So, $Z$ is not a $B$-inductive hypersurface.

But, as remarked in 6.2 , if $\operatorname{Sing}(B)$ has codimension at least two, any $B$-adapted hypersurface is automatically inductive, so for any $B$-adapted hypersurface the inductive object is defined. We shall use this observation several times, for instance in Sections 9.7 and 9.8.

It is well known that the object $B_{Z}$ is not necessarily nice, even if the base ring $A$ is a field $k$.

6.13. In case we are working over a field $k$ (characteristic zero), if $B=$ $(W, J, b, E)$ is a nice basic object and $Z$ an inductive hypersurface, it is proved in [11] that the object $B_{Z}$ has the following properties:

(i) $\operatorname{Sing}(B)=\operatorname{Sing}\left(B_{Z}\right)$. From this it follows that a subscheme $C$ of $W$ is a $B$-permissible center if and only if $C$ is a $B_{Z}$-permissible center.

(ii) If $B \leftarrow B_{1}$ is a permissible transformation, by $(i)$ using the same center we obtain a permissible transformation $B_{Z} \leftarrow\left(B_{Z}\right)_{1}$. By 6.8 the strict transform $Z_{1}$ of $Z$ to $B_{1}$ is an inductive hypersurface of $B_{1}$. Although we cannot prove that $\left(B_{1}\right)_{Z_{1}}$ is isomorphic to $\left(B_{Z}\right)_{1}$, it is true that $\operatorname{Sing}\left(\left(B_{1}\right)_{Z_{1}}\right)=\operatorname{Sing}\left(\left(B_{Z}\right)_{1}\right)$. We may repeat the process, taking a transformation of $B_{1}$ with a permissible center $C_{1}$ (which will also be $\left(B_{Z}\right)_{1}$ permissible.) After iterating $k$ times we get (in obvious notation):

$$
\text { (1) } \quad \operatorname{Sing}\left(\left(B_{k}\right)_{Z_{k}}\right)=\operatorname{Sing}\left(\left(B_{Z}\right)_{k}\right)
$$

The analog of $(i)$ in the context of basic objects over an artinian ring $A$ would be the assertion: if $B$ is a nice $A$-basic object and $Z$ is a $B$-inductive hypersurface, then a center $C$ is $B$-permissible if and only if it is $B_{Z}$-permissible. More precisely, if $C$ is a permissible center for $B_{Z}$, then by (A2) $C$ will be a permissible center for the pair $(W \rightarrow S, E)$ (see 3.5). But, will it be $B$-permissible (i.e., will $\nu(I, C)=\nu\left(I^{(0)}, C^{(0)}\right) \geq b$ hold?) Conversely, if $C$ is a $B$-permissible center, then by (A1) it will be permissible for the pair $\left(Z \rightarrow S, E_{Z}\right)$. But, will it be $B_{Z}$-permissible?

The answer to both questions is negative. Next we present examples.

Example 6.14. (Showing that $C$ is $B$-permissible does not imply $C$ is $B_{Z^{-}}$ permissible). Let $B=(W \rightarrow S, I, 2, \emptyset)$ where $S=\operatorname{Spec}(A), A=k[\epsilon], k$ a field, $\epsilon^{2}=0, W=\operatorname{Spec}(A[x, z]), I=\left(z^{2}+\epsilon x^{2}, z^{3}+x^{3}\right)$. Then, $\Delta^{1}(I / S)=$ $\left(z, \epsilon x, x^{2}\right)$ and one easily checks that $Z$, defined by the ideal $(z) A[x, z]$ is a $B$-inductive hypersurface, and $B_{Z}=\left(\operatorname{Spec}(A[x]) \rightarrow \operatorname{Spec}(A),\left(\epsilon x^{2}, x^{3}\right), 2, \emptyset\right)$. Then we see that $C \subset W$, defined by the ideal $(x, z)$ is a $B$-permissible center but not a $B_{Z}$-permissible center. 
Example 6.15. (Showing that $C$ is $B_{Z}$-permissible does not imply $C$ is $B$-permissible). Let $A$ and $W \rightarrow S$ be as in Example 6.14, $B=(W \rightarrow$ $S, I, 4, \emptyset)$, where $I=\left(x^{5}+\epsilon x^{2} z+z^{4}\right)$. Then one verifies that $Z$, the subscheme of $W$ defined by $(z) A[x, z]$ is a $B$-inductive hypersurface, and $B_{Z}=\left(\operatorname{Spec}(A[x]) \rightarrow \operatorname{Spec}(A),\left(x^{30}\right), 24, \emptyset\right)$. The subscheme $C$ of $Z$, defined by $(x) A[x]$ on $Z$ and by $(x, z)$ on $W$, is a $B_{Z}$-permissible center but not a $B$-permissible one.

Definition 6.16. We use the notation of 6.11. A closed subscheme $C$ of $Z$ is a strongly permissible center for $B_{Z}$ (or a strong $B_{Z}$-center) if it is a permissible center for both $B_{Z}$ and $B$.

6.17. We extend this notion to sequences as follows. Let $B$ be a nice $S$ basic object, $Z$ a $B$-inductive hypersurface. We use the notation of 6.11 . A permissible sequence of $S$-basic objects:

$$
B_{Z}:=\left(B_{Z}\right)_{0} \leftarrow\left(B_{Z}\right)_{1} \leftarrow \cdots \leftarrow\left(B_{Z}\right)_{m}
$$

(with centers $\left.C_{i} \subset u s\left(\left(B_{Z}\right)_{i}\right), i=0, \ldots m-1\right)$ is said to be strongly permissible if: $C_{0}$ is a strongly permissible $\left(B_{Z}\right)_{0}$-center (hence, $C_{0}$ is a $B$-center and if $B_{1}:=\mathcal{T}\left(B, C_{0}\right)$, us $\left(\left(B_{Z}\right)_{1}\right)$ may be identified, to the strict transform $Z_{1}$ of $Z$ to $u s\left(B_{1}\right)$, and $C_{1}$ to a closed subscheme of $\left.u s\left(B_{1}\right)\right), C_{1}$ is a $B_{1}$-permissible center; if $B_{2}:=\mathcal{T}\left(B_{1}, C_{1}\right)$, with similar identifications $C_{2}$ is a permissible $B_{2}$-center, and so on. So, eventually, with these identifications, $C_{i}$ must be a $B_{i}$ center, $i=0, \ldots, m-1$.

Thus, the strongly permissible sequence (1) induces a permissible sequence of $S$-basic objects

$$
\text { (2) } B \leftarrow B_{1} \leftarrow \cdots \leftarrow B_{m}
$$

using the same centers $C_{i}$ as in (1).

When $A=k=$ a field, according to 6.13 a center is permissible if and only if it is strongly permissible.

\section{The homogenized ideal and applications}

In this section we present, adapted to our needs, some notions and results due to Wlodarzcyck (cf. [28], where one works over a field). Throughout, $S=\operatorname{Spec}(A), A \in \mathcal{A}$, as in 3.1.

Definition 7.1. Given a scheme $W$, a weighted $W$-ideal is a pair $(I, b)$, where $I \subset \mathcal{O}_{W}$ is $W$-ideal and $b$ is a non-negative integer. Often, if $W$ is clear, we'll talk about a weighted ideal. 
Definition 7.2. Let $(I, b)$ be a weighted $W$-ideal, where $W$ is a scheme, smooth over $S$. The homogenized ideal, relative to $S$, associated to $(I, b)$ is the the $W$-ideal $\mathcal{H}(I / S, b)=I+\Delta(I / S) T(I / S)+\cdots+\Delta^{i}(I / S) T(I / S)^{i}+$ $\cdots+\Delta^{b-1}(I / S) T(I / S)^{b-1}$, where we have written $T(I / S):=\Delta^{b-1}(I / S)$. If $B=(W \rightarrow S, I, b, E)$ is a $A$-basic object, we use the notation $\mathcal{H}(B):=$ $(W \rightarrow S, \mathcal{H}(I / S, b), b, E)$ (a new $A$-basic object).

Note that passing to the fiber we obtain, in the usual notation, $\mathcal{H}\left(I^{(0)}, b\right)=$ $\mathcal{H}(I / S, b)^{(0)}$

Lemma 7.3. If $(I, b)$ is a weighted ideal on an $S$-smooth scheme $W$, then $\Delta^{b-1}(I / S)=\Delta^{b-1}(\mathcal{H}(I / S, b))$

The proof is elementary and we leave it to the reader.

Proposition 7.4. If $B=(W \rightarrow S, I, b, E)$ is a good A-basic object (see 5.4), then $\mathcal{H}(B)$ is $W$-equivalent to $B$.

Proof. By Remark 6.7 and Lemma 7.3 a center $C$ is $B$-permissible if and only if it is $\mathcal{H}(I)$-permissible. By induction, by the same reason it suffices to show that, for all $r>0$, if $B \leftarrow B_{1} \leftarrow \cdots \leftarrow B_{r}$ and $\mathcal{H}(B) \leftarrow[\mathcal{H}(B)]_{1} \leftarrow$ $\cdots \leftarrow[\mathcal{H}(B)]_{r}$ are permissible sequences of basic objects and transformations, where in both cases the same permissible centers have been used, then

$$
\text { (1) } \quad \Delta^{b-1}\left(I_{r} / S\right)=\Delta^{b-1}\left([\mathcal{H}(I / S, b)]_{r}\right)
$$

(where $\mathcal{H}(I / S, b)_{r}$ is the ideal in the basic object $\left.\mathcal{H}(B)_{r}\right)$. To simplify, sometimes we'll drop " $b$ ", writing, e.g., $\mathcal{H}(I / S, b)=\mathcal{H}(I / S)$.

First we shall check that

$$
\text { (2) } \quad[\mathcal{H}(I / S)]_{r} \subseteq \mathcal{H}\left(I_{r} / S\right)
$$

To see this, consider $\left[\mathcal{H}(I / S]_{r-1}=I_{r-1}+\sum_{i=1}^{b-1} \Delta^{i}\left(I_{r-1} / S\right) T\left(I_{r-1} / S\right)^{i}\right.$. The controlled transform of this ideal to $W_{r}$ is

$$
[\mathcal{H}(I / S)]_{r}=I_{r}+\sum_{i=1}^{b-1}\left[\Delta^{i}\left(I_{r-1} / S\right) T\left(I_{r-1} / S\right)^{i}\right]_{1} .
$$

But, letting $H$ be the exceptional divisor of the blowing up $W_{r-1} \leftarrow W_{r}$ and $\mathcal{E}:=I(H),\left[\Delta^{i}\left(I_{r-1} / S\right) T\left(I_{r-1} / S^{i}\right]_{1}=\mathcal{E}^{-(b-i)} \Delta^{i}(I / S)\left[\mathcal{E}^{-1} T(I / S)\right]^{i} \subseteq\right.$ $\Delta^{i}\left(I_{r} / S\right)\left[T\left(I_{r} / S\right)\right]^{i}$, the last inclusion by 6.9. From this (2) clearly follows. So, we have inclusions $I_{r} \subseteq \mathcal{H}(I / S, b)_{r} \subseteq \mathcal{H}\left(I_{r} / S, b\right)$. Applying the operator $\Delta^{b-1}$ to each of these and using 7.3 we see that the resulting first and third terms are equal, hence we obtain (1). 
We shall need the following basic result.

Proposition 7.5. Let $B=(W \rightarrow S, I, b, E)$ be an A-basic object, $y$ a point of $W, R=\mathcal{O}_{W, y}, a_{1}, \ldots, a_{r}$ an A-regular system of parameters of $R, M=$ $r(A)$ and $J=M R+\left(a_{1}, \ldots, a_{r}\right) R$. Then, $J$ is the maximal ideal of $R$.

Proof. Let $J^{\prime}:=\max (R)$. We have $J \subseteq J^{\prime}$. To see that equality holds, letting $R^{\prime}$ denote the completion of $R$ with respect to $J$, and using the fact that $R^{\prime}$ is a flat $R$-algebra, it suffices to show that $J R=J^{\prime} R^{\prime}$. Now, we have the following identifications: $R^{\prime} / J R^{\prime}=\left(R^{\prime} / M R^{\prime}\right) /\left(a_{1}, \ldots, a_{r}\right)\left(R^{\prime} / M R^{\prime}\right)=$ $(\widehat{R / M R}) /\left(a_{1}, \ldots, a_{r}\right)(\widehat{R / M R})=\widehat{\left(R^{(0)}\right)} / \max \widehat{\left(R^{(0)}\right)}$, where the hat indicates completion with respect to the maximal ideal. The last equality holds because $a_{1}, \ldots, a_{r}$ induces a regular system of parameters in the regular local $\operatorname{ring} R^{(0)}=\mathcal{O}_{W^{(0)}, y}$, in particular it generates the maximal ideal of $R^{(0)}$. So, $R^{\prime} / J R^{\prime}$ is a field, hence $J R^{\prime}$ must be equal to the maximal ideal $J^{\prime} R^{\prime}$, as claimed.

Proposition 7.6. With the notation of the previous proposition, letting $\widehat{R}$ denote the completion of $R$ with respect to its maximal ideal and $R^{\prime}$ the completion with respect to $\left(a_{1}, \ldots, a_{r}\right) R$, we have $R^{\prime}=\widehat{R}$.

Proof. Using the fact that $A$ is artinian and hence a power of its maximal ideal $M$ is equal to zero, it is easy to verify that there is a positive integer $s$ such that for all sufficiently large integers $t$ we have $Q^{t} \subseteq J^{t} \subseteq Q^{t-s}$, where $Q=\left(a_{1}, \ldots, a_{r}\right) R$ and $J=M R+\left(a_{1}, \ldots, a_{r}\right) R=r(R)$ (use 7.5). It is well known that these inclusions imply that the topologies defined by $Q$ and $J$ are the same, whence the claimed equality of completions.

Proposition 7.7. Let $B$, the point $y \in W$ and $R=\mathcal{O}_{W, y}$ be as in 7.5, let both $u, u_{2}, \ldots, u_{n}$ and $v, u_{2}, \ldots, u_{n}$ be A-regular systems of parameters of $R$, $\widehat{R}$ the completion of $R$ with respect to its maximal ideal $\mathcal{M}$. Then, there is an A-linear automorphism $\phi$ of $\widehat{R}$ such that $\phi(u)=v$ and $\phi\left(u_{i}\right)=u_{i}$ for $i=2, \ldots, n$ and, moreover, such that if $h=u-v$ and $p: \widehat{R} \rightarrow \widehat{R} /(h)$ is the canonical quotient homomorphism, then $p \phi=p$.

Proof. Consider the completion $R^{*}$ of $R$ with respect to the ideal generated by $\left(u, u_{2}, \ldots, u_{n}\right)$. We know: (i) $R^{*}=A^{\prime}\left[\left[u, u_{2}, \ldots, u_{n}\right]\right]$, for a suitable overring $A^{\prime}$ of $A$ (see 3.8 or 11.6), (ii) $R^{*}=\widehat{R}$ (see 7.6.) Using the identification (ii), to prove the proposition it suffices to define an automorphism $\phi$ of $R^{*}$ satisfying the required properties. Define $\phi$ by the conditions: $\phi(a)=a$ for $a \in A^{\prime}, \phi(u)=v$ and $\phi\left(u_{j}\right)=u_{j}, j=2, \ldots, n$. This is correct, since $v$, regarded as an element of $R^{*}$, must be of the form $v=m+\alpha$, with $m \in M R^{*}(M=r(A)), \alpha \in\left(u, u_{2}, \ldots, u_{n}\right) R^{*}$, and $M^{s}=0$ for $s$ large enough. Modulo $M R^{*}$, this induces a homomorphism of rings of formal power series $K\left[\left[u, u_{2}, \ldots, u_{n}\right]\right] \rightarrow K\left[\left[v, u_{2}, \ldots, u_{n}\right]\right]$ (with $K$ a field), which 
clearly is an isomorphism. By the "nilpotent" Nakayama's Lemma, $\phi$ must be an isomorphism. The statement about the quotient map $p$ follows from the fact that $u-\phi(u)=h$.

Proposition 7.8. Keep the assumptions and notation of 7.7, but also assume that $u \in T(I / S)_{y}$ and $v \in T(I / S)_{y}$. Then, the automorphism $\phi$ satisfies: $\phi(\mathcal{H}(I / S) \widehat{R})=\mathcal{H}(I / S) \widehat{R}$.

Proof. Above, we wrote $\mathcal{H}(I / S) \widehat{R}:=\mathcal{H}(I / S)_{y} \widehat{R}$. Recall the definitions in 7.7: we identify $\widehat{R}$ with $R^{*}:=A^{\prime}\left[\left[u, u_{2}, \ldots, u_{n}\right]\right]$, then $\phi: R^{*} \rightarrow R^{*}$ is defined by: $\phi(u)=v, \phi\left(u_{i}\right)=u_{i}$, for $i=2, \ldots, n, \phi(a)=a$ for $a \in A^{\prime}$. Thus, for $f\left(u, u_{2}, \ldots, u_{n}\right) \in R^{*}$,

$$
\phi f=f(u)+\frac{\partial f}{\partial u} h+\frac{1}{2 !} \frac{\partial^{2} f}{\partial u^{2}} u^{2} h^{2}+\frac{1}{3 !} \frac{\partial^{3} f}{\partial u^{3}} u^{3} h^{3}+\cdots
$$

(With this set-up, what follows imitates the proof of [28], Lemma 2.9.4). To check the desired equality, since $\phi$ is an automorphism of a noetherian ring, it suffices to show: $\phi\left(\mathcal{H}(I / S) R^{*}\right) \subseteq \mathcal{H}(I / S) R^{*}$.

To check the latter inclusion, in view of the definition of $\mathcal{H}(I / S)$ in 7.2, it suffices to show: $\phi\left(\Delta^{j}(I / S)[T(I / S)]^{j} R^{*} \subseteq \mathcal{H}(I / S) R^{*}, j=0, \ldots, b-1\right.$, where as in 7.2 we have written $T(I / S)=\Delta^{b-1}(I / S)$. It is easy to verify that this inclusion follows if we can prove the following assertions: $\left(a_{j}\right)$ : $\phi\left(\Delta^{j}(I / S) R^{*}\right) \subseteq \mathcal{H}\left(\Delta^{j}(I / S) R^{*}\right), j=0, \ldots, b-1,(b): \phi\left(T(I / S) R^{*}\right) \subseteq$ $\mathcal{H}(I / S) R^{*}$. Now, $\left(a_{0}\right)$ is a consequence of formula (1) above. Since this is valid for any ideal $I$, we may substitute (in $\left.\left(a_{0}\right)\right) I$ by $\Delta^{j}(I / S)$, and we obtain $\left(a_{j}\right)$, for all $j$. Concerning $(b)$, if $g \in T(I / S)_{y}$, by (1) (with $f=g$ ) and the fact that $h=u-v \in T(I / S)$ (hence $h^{i} \in\left[T(I / S)_{y}\right]^{i}$ for all $i$ ), it follows that $\phi(g) \in T(I / S)$, as desired.

Let us recall some terminology and notation to be used in the next theorem. Given a scheme $W$ and a point $w \in W$, an etale neighborhod of $y$ in $W$ consists of a scheme $V$, a point $v \in V$ and an etale morphism $\pi: V \rightarrow W$ such that $\pi(v)=w$. We use the notation $\pi:(V, v) \rightarrow(W, w)$ to indicate this etale neighborhood. To shrink the etale neighborhood $\pi$ means to take the composition of $\pi \pi^{\prime}$ of $\pi$ with another etale neighborhood $\pi^{\prime}:\left(V^{\prime}, v^{\prime}\right) \rightarrow(V, v)$. If $f$ and $g$ are morphisms from a scheme $V$ to a scheme $W$ and $Y$ is a closed subscheme of $W$, we say that $f$ and $g$ agree over $Y$ if $f^{-1}(Y)=g^{-1}(Y):=Y^{\prime}$ and $f j=g j$, where $j: Y^{\prime} \rightarrow V$ is the inclusion.

If $B=(p: W \rightarrow S, I, b, E)$ is an $S$-basic object, $f: V \rightarrow W$ an etale morphism, $f^{*}(B)$ denotes the $S$-basic object $\left(V, f^{*}(I), b, f^{*}(E)\right)$, where $f^{*}(I):=I \mathcal{O}_{V}$ and $f^{*}(E)$ is the sequence of inverse images by $f$ of the hypersurfaces that appear in $E$. 
Theorem 7.9. Let $B=(W \rightarrow S, I, b, E)$ be a nice basic object, $Z$ and $Z^{\prime}$ adapted hypersurfaces, $y \in Z \cap Z^{\prime}$. Let $Z$ (resp. $Z^{\prime}$ ) be defined by $u \in$ $\Gamma\left(W, \mathcal{O}_{W}\right)$ (resp. $v \in \Gamma\left(W, \mathcal{O}_{W}\right)$ ) and $J=\mathcal{H}(I / S)$. Then, there are etale neighborhoods $\pi_{u}:(V, z) \rightarrow(W, y)$ and $\pi_{v}:(V, z) \rightarrow(W, y)$ such that: (a) $\pi_{u}^{*}(J)=\pi_{v}^{*}(J),(b) \pi_{u}^{*}(u)=\pi_{v}^{*}(v)$, (c) if $H$ is any hypersurface in $E$, then $\pi_{u}^{-1}(H)=\pi_{v}^{-1}(H)$ (hence, $\left.\pi_{u}^{*}(E)=\pi_{v}^{*}(E)\right),(d) \pi_{u}=\pi_{v}$ over $\mathcal{V}(u-v)$.

Proof. Since $W$ is of finite type over $A$, we may find an affine open neighborhood of $y$, still denoted by $W$, of the form $\operatorname{Spec}(D)$, where $D=A[\mathbf{x}] / K$, with $\mathbf{x}=\left(x_{1}, \ldots, x_{m}\right)\left(x_{1}, \ldots, x_{m}\right.$ algebraically independent over $\left.A\right)$ and $K$ and ideal of $A[\mathbf{x}]$, say generated by $f_{i} \in A[\mathbf{x}], i=1, \ldots, r$. The point $y$ corresponds to a prime ideal $P$ of $D$, so we may identify $R=\mathcal{O}_{W, y}=D_{P}$. Moreover, shrinking $W$, if necessary, we may assume that: $(i) J R=\left(g_{1}, \ldots, g_{t}\right) R$, where $g_{j} \in A[\mathbf{x}]$, for all $j,(i i)$ there are elements $u_{2}, \ldots, u_{n}$ in $R$ such that both $u, u_{2}, \ldots, u_{n}$ and $v, u_{2}, \ldots, u_{n}$ are $A$-regular systems of parameters of $R$, where each hypersurface $H$ in $E$ containing $y$ is defined at $y$ by some $u_{i}$, $i>1$ and, moreover, $u$ and $u_{j}, j=2, \ldots, n$ are images in $R=D_{P}$ of polynomials (in $A[\mathbf{x}]) q(\mathbf{x})$ and $q_{j}(\mathbf{x}), j=2, \ldots, n$ respectively.

Now, we have natural homomorphisms

$$
A[\mathbf{x}] \rightarrow A[\mathbf{x}] / K \rightarrow(A[\mathbf{x}] / K)_{P}=R \rightarrow \widehat{R}
$$

where $\widehat{R}$ denotes the completion of $R$ with respect to its maximal ideal. Let $\psi: A[\mathbf{x}] \rightarrow \widehat{R}$ be the composition of these homomorphisms, and $a_{i}$ the image of $x_{i} \in A[\mathbf{x}]$ in $\widehat{R}, i=1, \ldots, m$. The fact that $\psi$ factors through $K$ means:

$$
f_{i}\left(a_{1}, \ldots, a_{m}\right)=0, i=1, \ldots, r
$$

Now consider the automorphism $\phi$ (of $\widehat{R}$ ) associated to $u, v, u_{2}, \ldots, u_{n}$, as in 7.7, and the composition $\phi \psi: A[\mathbf{x}] \rightarrow \widehat{R}$. Since $\phi$ is $A$-linear and the coefficients of $q(\mathbf{x})$ are in $A$, the fact that $\phi(u)=v$ means:

$$
q\left(a_{1}, \ldots, a_{m}\right)=v \in R
$$

For the same reasons, the fact that $\phi\left(u_{i}\right)=u_{i}, i=2, \ldots, n$, means

$$
q_{i}\left(a_{1}, \ldots, a_{m}\right)=u_{i} \in R, i=2, \ldots, n
$$

Let $J R$ be generated by elements $h_{1}, \ldots, h_{p}$ in $R$. Then, as above, from $\phi(J R)=J R$ we obtain:

$$
g_{j}=c_{j 1} h_{1}+\cdots, c_{j p} h_{p}, j=1, \ldots, t
$$

for suitable elements $c_{j i} \in \widehat{R}$. 
We may view (1) through (4) as polynomial equations with coefficients in $R$ in unknowns $\left\{A_{k}\right\}$ and $\left\{C_{j i}\right\}$, which have a solution $\left\{a_{k}\right\}$ and $\left\{c_{j i}\right\}$ in $\widehat{R}$. By Artin's approximation Lemma (see [2] 1.10, note that since $A$ is a finite dimensional vector space over $k=A / r(A)$, the hypothesis are valid) for any positive integer $s$ we find solutions $\left\{b_{k}\right\},\left\{d_{j i}\right\}$ in $\widetilde{R}$, the henselization of $R$ with respect to its maximal ideal. Choose $s \geq 2$. By sending $X_{i}$ to $b_{i} \in \widetilde{R} \subset$ $\widehat{R}, i=1, \ldots, m$, we obtain an $A$-linear homomorphism $\alpha^{\prime}: A[\mathbf{x}] \rightarrow \widetilde{R} \subset \widehat{R}$, which induces a homomorphism $\alpha: A[\mathbf{x}] / K \rightarrow \widetilde{R}$ and, localizing at $P$ and completing, another $\phi^{\prime}: \widehat{R} \rightarrow \widehat{R}$. By our choice $s \geq 2$, $\phi^{\prime}$ agrees with the isomorphism $\phi$ modulo $\mathcal{N}^{2}\left(\mathcal{N}=r(\widehat{R})\right.$. Hence, $\phi^{\prime}$ is also an automorphism of $\widehat{R}$, satisfying $\phi^{\prime}(u)=v$ and $\phi^{\prime}\left(u_{i}\right)=u_{i}, i=1, \ldots, n$.

Returning to $\alpha: D \rightarrow \widetilde{R}$, from the definition of henselization (involving a limit of etale neighborhoods) for some etale neighborhood $\pi_{v}:(V, z) \rightarrow$ $(W, y)$ there is an induced morphism $\bar{\alpha}: V \rightarrow W$ such that $\bar{\alpha}(z)=y$. If we consider the induced homomorphism $\widehat{\mathcal{O}_{W, y}} \rightarrow \widehat{\mathcal{O}_{V, z}}$, by our construction this may be identified to the isomorphism $\phi^{\prime}: \widehat{R} \rightarrow \widehat{R}$. Thus, $\bar{\alpha}$ is etale at $z$. Hence, shrinking $V$ if necessary, $\bar{\alpha}$ defines an etale neighborhood $\pi_{u}:(V, z) \rightarrow(W, y)$. Shrinking again this neighborhood if necessary, by using 7.7 we see $\pi_{u}$ and $\pi_{v}$ satisfy properties (a) through (d).

7.10. Theorem 7.9 can be extended to sequences of permissible transformations of $A$-basic objects as follows. Suppose we are in the conditions of 7.9. We retain the notation of that theorem, but we also write $B_{0}:=(W \rightarrow$ $S, J, b, E)$ (recall $J=\mathcal{H}(I / S))$. Take a $B_{0}$-permissible center $C$. Since $C \subseteq$ $\mathcal{V}(u-v)$, by $7.9(\mathrm{~d}) \pi_{u}^{-1}(C)=\pi_{u}^{-1}(C):=\bar{C} \subseteq V$. Let us write $\bar{J}:=\pi_{u}^{*}(J)=$ $\pi_{v}^{*}(J)$ (the equality by $\left.7.9,(\mathrm{a})\right)$, and $\bar{B}_{0}=(V, \bar{J}, b, \bar{E})$, where $\bar{E}:=\pi_{u}^{*}(E)=$ $\pi_{v}^{*}(E)$. Since $\pi_{u}$ and $\pi_{v}$ are etale, we have that both $\pi_{u}^{*}\left(\Delta^{b-1}(J / S)\right)$ and $\pi_{u}^{*}\left(\Delta^{b-1}(J / S)\right)$ agree with $\Delta^{b-1}(\bar{J} / S)$, i.e, $\pi_{u}^{*}\left(\Delta^{b-1}(J / S)\right)=\pi_{u}^{*}\left(\Delta^{b-1}(J / S)\right)$ $=\Delta^{b-1}(\bar{J} / S)$. Now we may easily check that $\bar{C}$ is a $\bar{B}$-permissible center. We transform $B$ and $\bar{B}$ with centers $C$ and $\bar{C}$ respectively, getting $B \leftarrow B_{1}=\left(W_{1} \rightarrow S, J_{1}, b, E_{1}\right)$ and $\bar{B}_{0} \leftarrow \bar{B}_{1}=\left(\bar{W}_{1}, \bar{J}_{1}, b, \bar{E}_{1}\right)$ respectively. By Proposition 11.7, both the pull-backs of $\pi_{u}: V \rightarrow W$ and $\pi_{v}: V \rightarrow W$ via $W \leftarrow W_{1}$ (the blowing-up of $W$ with center $C$ ) may be identified to the blowing-up $V_{1}$ of $V$ with center $\bar{C}$. Hence we get etale morphisms $\pi_{1 u}, \pi_{1 v}$ from $V_{1}$ to $W_{1}$ induced by $\pi_{u}$ and $\pi_{v}$ respectively. Notice that again, by the etaleness, both $\pi_{1 u}^{*}\left(J_{1}\right)$ and $\pi_{1 v}^{*}\left(J_{1}\right)$ may be identified to $\bar{J}_{1}$, hence they are equal. Similarly, if $u_{1}$ (resp. $v_{1}$ ) define the strict transform of $Z$ (resp. $Z^{\prime}$ ) to $W_{1}$, then $\pi_{1 u}^{*}\left(u_{1}\right)=\pi_{1 v}^{*}\left(v_{1}\right)=\bar{u}_{1}$, where $\bar{u}_{1}$ is the proper transform of $\bar{u}$ to $V_{1}$. Note that (by 6.8) these hypersurfaces are again adapted.

A formal argument with cartesian (i.e., fiber product) squares shows that $\pi_{1 u}=\pi_{1 v}$ over $\mathcal{V}\left(u_{1}-v_{1}\right)$ (in the sense explained after the statement of 7.9) 
and moreover, if we choose a point $y_{1} \in W_{1}$ lying over $y_{0}:=y$, then there is a point $z_{1} \in V_{1}$ lying over $z_{0}$ such that $\pi_{u 1}\left(z_{1}\right)=\pi_{v 1}\left(z_{1}\right)=y_{1}$.

Now the procedure may be iterated: if $C_{1}$ is a permissible $B_{1}$-center, then $\pi_{1 u}^{-1}\left(C_{1}\right)=\pi_{1 v}^{-1}\left(C_{1}\right):=\overline{C_{1}}$ and this is a permissible center for $\bar{B}_{1}$, we take the transforms of $B_{1}$ and $\bar{B}_{1}$ with centers $C_{1}$ and $\overline{C_{1}}$ respectively, and proceed as before. Iterating, we obtain the following result:

Theorem 7.11. Let

$$
\text { (1) } \quad B_{0} \leftarrow B_{1} \leftarrow \cdots \leftarrow B_{r}
$$

be a permissible sequence of $A$-basic objects and transformations, where $B_{0}$ is the homogenized basic object associated to a nice basic object $B=(W \rightarrow$ $S, I, b, E)$, we write $B_{i}=\left(W_{i} \rightarrow S, J_{i}, b, E_{i}\right)\left(s o, J_{0}=\mathcal{H}(I / S), W_{0}=W\right)$, $p_{i}: W_{i} \rightarrow W_{i-1}$ the corresponding blowing-up morphism, $i=1, \ldots, r$. Let $Z$ and $Z^{\prime}$ be $B$-adapted hypersurfaces, defined by elements $u$, $v$ of $\Gamma\left(W, \mathcal{O}_{W}\right)$ respectively, $y_{0} \in Z \cap Z^{\prime}$ a point of $W$. Then there are a permissible sequence of A-basic objects and transformations $\overline{B_{0}} \leftarrow \overline{B_{1}} \leftarrow \cdots \leftarrow \overline{B_{r}}$ (we write $\overline{B_{i}}=\left(V_{i} \rightarrow S, \overline{J_{i}}, b, \overline{E_{i}}\right), q_{i}$ denotes the $i$-th blowing-up morphism $\left.V_{i} \rightarrow V_{i-1}\right)$, and for each $i$ etale morphisms $\pi_{i u}$ and $\pi_{i v}$ from $V_{i}$ to $W_{i}$ such that:

(i) $\pi_{i u}^{*}\left(B_{i}\right)=\overline{B_{i}}=\pi_{i v}^{*}\left(B_{i}\right)$, in particular $\pi_{i u}^{*}\left(J_{i}\right)=\pi_{i v}^{*}\left(J_{i}\right)$

(ii) $\pi_{i u}^{*}\left(u_{i}\right)=\pi_{i v}^{*}\left(v_{i}\right)$, where $u_{i}$ and $v_{i}$ denote the strict transforms of $u$ and $v$ to $W_{i}$, respectively.

(iii) $\pi_{i u}=\pi_{i v}$ over $\mathcal{V}\left(u_{i}-v_{i}\right)$

(iv) for all $i, \pi_{i u}^{-1}\left(C_{i}\right)=\pi_{i v}^{-1}\left(C_{i}\right)$ and this is the center of the $i$-th transformation $\overline{B_{i}} \leftarrow \overline{B_{i+1}}$

(v) for all $i$, the squares

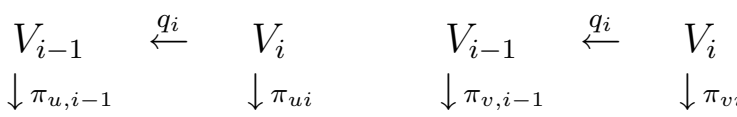

$$
\begin{aligned}
& W_{i-1} \stackrel{p_{i}}{\longleftarrow} W_{i} \quad W_{i-1} \stackrel{p_{i}}{\leftarrow} W_{i}
\end{aligned}
$$

are cartesian.

(vi) If $y_{i} \in W_{i}$ is such that $p_{i}\left(y_{i}\right)=y_{i-1}, i=1, \ldots, r$, then there are points $z_{i} \in V_{i}, i=1, \ldots, r$, such that $\pi_{u i}\left(z_{i}\right)=\pi_{v i}\left(z_{i}\right)=y_{i}$ and $q_{i}\left(z_{i}\right)=$ $z_{i-1}, C$. Moreover, if $z_{0}, \ldots, z_{r-1}$ as above are given, then we can find $z_{r} \in V_{r}$, lying over $z_{r-1}$ such that $\pi_{u r}\left(z_{r}\right)=\pi_{v r}\left(z_{r}\right)=y_{r}$ and $q_{r}\left(z_{r}\right)=z_{r-1}$

7.12. Next we discuss a version of 7.11 relative to inductive objects. In it, we use the following notation and assumptions. Let $B_{0}=\left(W_{0} \rightarrow S, J_{0}, b, E_{0}\right)$ be as in 7.11, i.e., the homogenized $A$-basic object associated to a nice basic object $B=\left(W_{0} \rightarrow S, I, b, E\right), Z$ and $Z^{\prime}$ be $B$-inductive hypersurfaces, 
defined by elements $u, v$ of $\Gamma\left(W_{0}, \mathcal{O}_{W_{0}}\right)$ respectively. Let $C_{0} \subset Z \cap Z^{\prime}$ be a strongly permissible center for both $B_{0}^{*}:=\left(B_{0}\right)_{Z}$ and $B^{\prime *}{ }_{0}:=\left(B_{0}\right)_{Z^{\prime}}$. Hence, writing $B_{0}:=B, C_{0}$ is also a $B_{0}$-permissible center. Consider $B_{1}^{*}=$ $\mathcal{T}\left(B_{0}^{*}, C_{0}\right), B_{1}^{\prime *}=\mathcal{T}\left(B_{0}^{\prime *}, C_{0}\right)$ and $B_{1}=\mathcal{T}\left(B_{0}, C_{0}\right)$. By 6.8 we may identify $u s\left(B_{1}^{*}\right)$ and $u s\left(B_{1}^{\prime *}\right)$ with the strict transforms $Z_{1}$ and $Z_{1}^{\prime}$ of $Z_{0}:=Z$ and $Z_{0}^{\prime}:=Z^{\prime}$ to $W_{1}=u s\left(B_{1}\right)$ (via the blowing-up $W_{1} \rightarrow W$ with center $C_{0}$ ) respectively; again $Z_{1}$ and $Z_{1}^{\prime}$ will be $B_{1}$-inductive hypersurfaces. Let $C_{1} \subset$ $Z_{1} \cap Z_{1}^{\prime}$ be a permissible center simultaneously for $B_{1}^{*}, B_{1}^{\prime *}$ and $B_{1}$. Transform these objects with center $C_{1}$, obtaining $A$-basic objects $B_{2}^{*}, B_{2}^{\prime *}$ and $B_{2}$ respectively. Again by 6.8 we may identify us $\left(B_{2}^{*}\right)=Z_{2}, u s\left(B_{2}^{\prime *}\right)=Z_{2}^{\prime}$, where $Z_{2}$ and $Z_{2}^{\prime}$ are the strict transforms of $Z_{1}$ and $Z_{1}^{\prime}$ to $W_{2}=u s\left(B_{2}\right)$, and so on. We assume this can be repeated, eventually obtaining strongly permissible sequences of $A$-basic objects (6.17):

$$
\left(B_{0}\right)_{Z}=B^{*}{ }_{0} \leftarrow \cdots \leftarrow B^{*}
$$

and

$$
\left(B_{0}\right)_{Z}=B^{\prime *}{ }_{0} \leftarrow \cdots \leftarrow B^{*}{ }_{r}
$$

where in each case we have used (in the sense just explained) the same centers $C_{i} \subset Z_{i} \cap Z_{i}^{\prime}$, where $Z_{i}=u s\left(B^{*}{ }_{i}\right), Z_{i}^{\prime}=u s\left(B^{* *}{ }_{i}\right), i=0, \ldots, r-1$, as well as one:

$$
\text { (3) } \quad B_{0} \leftarrow \cdots \leftarrow B_{r}
$$

where the center of $B_{i} \leftarrow B_{i+1}$ is again $C_{i}$. Since, for all $i, Z_{i}$ and $Z_{i}^{\prime}$ are $B_{i}$-inductive hypersurfaces in $W_{i}$, it makes sense to take a point $y \in Z_{i} \cap Z_{i}^{\prime}$. We still denote by $p_{i}$ the morphisms $Z_{i} \rightarrow Z_{i-1}$ and $Z_{i}^{\prime} \rightarrow Z_{i-1}^{\prime}$ induced by $p_{i}: W_{i} \rightarrow W_{i-1}$ of 7.11. Let $\pi_{i}: Y_{i} \rightarrow Z_{i}$ and $\pi_{i}^{\prime}: Y_{i} \rightarrow Z_{i}^{\prime}$ be the morphisms induced by $\pi_{u, i}$ and $\pi_{v, i}$ respectively, $i=0, \ldots, r$. Then we have:

Theorem 7.13. The hypotheses and notation are those of 7.11 and 7.12, so we have the sequences (1), (2), (3) there introduced. Then, there is a permissible sequence of $A$-basic objects and transformations $\tilde{B}_{0} \leftarrow \cdots \leftarrow \tilde{B}_{r}$ (we write $\tilde{B}_{i}=\left(Y_{i} \rightarrow S, \tilde{J}_{i}, b !, \tilde{E}_{i}\right), q_{i}^{*}: Y_{i} \rightarrow Y_{i-1}$ the induced morphism, for all $i$ ), and for each $i$ etale morphisms $\pi_{i}: Y_{i} \rightarrow Z_{i}, \pi_{i}^{\prime}: Y_{i} \rightarrow Z_{i}^{\prime}$, such that $\pi_{i}{ }^{*}\left(B^{*}{ }_{i}\right)=\tilde{B}_{i}=\pi_{i}^{\prime *}\left(B^{\prime *}\right)$ and the squares

$$
\begin{aligned}
& Y_{i-1} \stackrel{q^{*}{ }_{i}}{\longleftarrow} \quad Y_{i} \quad Y_{i-1} \stackrel{q^{*}{ }_{i}}{\longleftarrow} Y_{i} \\
& \downarrow \pi_{i-1} \quad \downarrow \pi_{i} \quad \downarrow \pi_{i-1}^{\prime} \quad \downarrow \pi_{i}^{\prime} \\
& Z_{i-1} \stackrel{p_{i}}{\leftarrow} Z_{i} \quad Z_{i-1}^{\prime} \stackrel{p_{i}}{\leftarrow} Z_{i}^{\prime}
\end{aligned}
$$

are cartesian.

Moreover, if $y_{i} \in Z_{i} \cap Z_{i}^{\prime}$ is such that $p_{i}\left(y_{i}\right)=y_{i-1}, i=1, \ldots, r$ and $z_{i} \in$ $Y_{i}, i=1, \ldots, r-1$ is such that $\bar{\pi}_{i}\left(z_{i}\right)=\bar{\pi}_{i}^{\prime}\left(z_{i}\right)=y_{i}$ and $q^{*}{ }_{i}\left(z_{i}\right)=z_{i-1}$, then there is a point $z_{r} \in V_{r}$, such that $q_{r}^{*}\left(z_{r}\right)=z_{r-1}$ and $\pi_{r}\left(z_{r}\right)=\pi_{r}^{\prime}\left(z_{r}\right)=y_{r}$. 
Proof. Let $Y_{0}:=\pi_{u, 0}{ }^{-1}\left(Z_{0}\right)=\pi_{v, 0}{ }^{-1}\left(Z_{0}^{\prime}\right)$. Then, the induced morphisms $\pi_{0}: Y_{0} \rightarrow Z_{0}$ and $\pi_{0}^{\prime}: Y_{0} \rightarrow Z_{0}^{\prime}$ are etale. From this fact, $\pi_{0}{ }^{*}\left(B^{*}{ }_{0}\right)=$ $\pi_{0}^{\prime *}\left(B^{\prime *}{ }_{0}\right)=\overline{B_{0}} Y_{0}$ and $\overline{C_{0}}$ is a strongly permissible center for the inductive object $\overline{B_{0}} Y_{0}$. Let $\left(\overline{B_{0} Y_{0}}\right)_{1}:=\mathcal{T}\left(\overline{B_{0}} Y_{0}, \overline{C_{0}}\right)(6.11(\mathrm{~b}))$. Then, using the notation of $7.10, Y_{1}:=u s\left(\left(\overline{B_{0} Y_{0}}\right)_{1}\right)$ may be identified to the hypersurface of $V_{1}$ defined by $\bar{u}$ and $\overline{C_{1}}$ to a closed subscheme of $Y_{1}$. The induced morphisms $\pi_{1}$ : $Y_{1} \rightarrow Z_{1}$ and $\pi_{1}^{\prime}: Y_{1} \rightarrow Z_{1}^{\prime}$ are again etale, which implies that $\pi_{1}{ }^{*}\left(B^{*}{ }_{1}\right)=$ $\pi_{1}^{\prime *}\left(B^{\prime *}{ }_{1}\right)=\left(\overline{B_{0} Y_{0}}\right)_{1}$ and $\overline{C_{1}}$ is a permissible center for both $\left(\overline{B_{0}} Y_{0}\right)_{1}$ and $\overline{B_{1}}$. Continuing in this way, we get a permissible sequence of $A$-basic objects

$$
\overline{B_{0} Y_{0}} \leftarrow\left(\overline{B_{0}} Y_{0}\right)_{1} \leftarrow \cdots \leftarrow\left(\overline{B_{0}} Y_{0}\right)_{r}
$$

obtained by using the centers $\overline{C_{i}}, i=0, \ldots, r-1$ of 7.11 . Let $Y_{i}:=u s\left(\left(\overline{B_{0}} Y_{0}\right)_{i}\right)$, $i=0, \ldots r$. Then $\pi_{i}: Y_{i} \rightarrow Z_{1}$ and $\pi_{i}^{\prime}: Y_{1} \rightarrow Z_{i}^{\prime}$ (the morphisms induced by $\pi_{u, i}$ and $\left.\pi_{v, i}\right)$, respectively) are etale, and $\pi_{i}^{*}\left(B^{*}{ }_{i}\right)=\left(\overline{B_{i}}\right)_{Y_{i}}=\pi_{i}^{\prime *}\left(B^{\prime *}{ }_{i}\right)$, for all $i=1, \ldots, r$. The other assertions are consequences of 7.11 .

\section{The associated locally nice object}

In this section we discuss how to associate to a basic object $B$ over an artinian local ring $A \in \mathcal{A}$, locally, a nice object $B^{\prime \prime}$, extending the results of $2.9(\gamma)$. The presentation follows that of [11], where one works over a base field. In particular, the construction of $B^{\prime \prime}$ is done via an intermediate object $B^{\prime}$, which has some but not all the desired properties. Since the discussion in [11] is rather sketchy, and some changes are necessary in the present context, we provide some details.

Throughout this section, $A \in \mathcal{A}$ is an artinian ring as in 3.1, $S=$ $\operatorname{Spec}(A)$. Given an $A$-basic object $B=(W \rightarrow S, I, b, E)$, to say " $C$ is a center of $B$ " means: $C$ is a center for the underlying pair $(W \rightarrow S, E)$, see 3.5. (To be a $B$-center is stronger, see 4.2 ).

We shall use the notation and results of 2.7 and 5.1 about the functions $\omega_{r}$ and $t_{r}$.

Definition 8.1. Consider a $\omega$-permissible $A$-sequence $B_{0} \leftarrow \cdots \leftarrow B_{r}$, let $\max \left(\omega_{r}\right)=b_{r} / b$. We associate to $B_{r}=\left(W_{r} \rightarrow S, I_{r}, b, E_{r}\right)$ the following basic object $B_{r}^{\prime}$. There are two cases:

$(\alpha) b_{r} \geq b$. We define $B_{r}^{\prime}=\left(W_{r} \rightarrow S, \overline{I_{r}}, b_{r}, E_{r}\right)$ (see 4.5 for $\overline{I_{r}}$ ),

( $\beta) b_{r}<b$. In the notation of Proposition 5.3, let $E_{r}=\left(H_{1}, \ldots, H_{m+r}\right)$ (where $H_{1}, \ldots, H_{m}$ are the strict transforms of the hypersurfaces that appear in $\left.E_{0}\right), \mathcal{C}_{r}=I\left(H_{m+1}\right)^{a_{1}} \ldots I\left(H_{m+r}\right)^{a_{r}}$; we set $B_{r}^{\prime}=\left(W_{r} \rightarrow S, \bar{I}_{r}^{b-b_{r}}+\right.$ $\left.\mathcal{C}^{b_{r}}, b_{r}\left(b-b_{r}\right), E_{r}\right)$. 
Proposition 8.2. With notation and assumptions as in 8.1, the A-basic object $B^{\prime} r=\left(W_{r} \rightarrow S, J_{r}, b^{\prime}, E_{r}\right)$ has the following properties:

(a) $\left(B_{r}^{\prime}\right)^{(0)}=\left(B_{r}^{(0)}\right)^{\prime}$

(b) A center $C \subset W_{r}$ is $\omega$-permissible (see 5.1) if and only if it is $B_{r}^{\prime}$ permissible.

(c) For every $B_{r}^{\prime}$-center $C, \nu(J, C)=\nu\left(J^{(0)}, C^{(0)}\right)=b^{\prime}$, where $b^{\prime}$ is the index of $B_{0}^{\prime}$.

(d) Let $C \subset W_{r}$ be a center which is $\omega$-permissible (or $B_{r}^{\prime}$-permissible, by $(b)$ it is the same). Consider the transformations $B_{r}^{\prime} \leftarrow\left(B_{r}^{\prime}\right)_{1}$ and $B_{r} \leftarrow$ $B_{r+1}$ with center $C$, and the associated object $B_{r+1}^{\prime}$. Assume $\max \left(\omega_{r}\right)=$ $\max \left(\omega_{r+1}\right)$. Then, $\left(B_{r}^{\prime}\right)_{1}=B_{r+1}^{\prime}$

Proof. (a) This readily follows from the definitions.

(b) From [11] (or by direct verification, which is easy) we have:

$$
\nu\left({\overline{I_{r}}}^{(0)}, C^{(0)}\right)=b_{r}
$$

Thus, to prove (b) we have to show: for a center $C, \nu\left(\overline{I_{r}}, C\right)=\nu\left(\overline{I_{r}}, C\right) \geq$ $b_{r} \Leftrightarrow \nu\left(J_{r}, C\right)=\nu\left(J_{r}, C\right) \geq b^{\prime}$. In case $(\alpha), J_{r}=\overline{I_{r}}$, so the equivalence is obvious (and the $\geq$ symbols are equalities). So, consider case $(\beta)$, where $J_{r}=\left(\overline{I_{r}}\right)^{b-b_{r}}+\mathcal{C}_{r}^{b_{r}}$ and $b^{\prime}=b_{r}\left(b-b_{r}\right)$.

Check the implication $\Rightarrow$ first. By our assumption and (1), we have $\nu\left({\overline{I_{r}}}^{b-b_{r}}, C\right)=\nu\left({\overline{I_{r}}}^{(0)^{b-b_{r}}}, C^{(0)}\right)=b_{r}\left(b-b_{r}\right)$. On the other hand, since our sequence of $A$-basic objects of 8.1 is permissible, $\nu\left(\mathcal{C}_{r}, C\right)=\nu\left(\mathcal{C}_{r}^{(0)}, C^{(0)}\right)=$ $b-b_{r}$ (the exponents $a_{i}$ are the same over $A$ or at the level of the special fiber). Then $\nu\left(\mathcal{C}_{r}^{b_{r}}, C\right)=\nu\left(\left(\mathcal{C}_{r}^{(0)}\right)^{b_{r}}, C^{(0)}\right)=b_{r}\left(b-b_{r}\right)$ and the implication $\Rightarrow$ follows.

Now we check the implication $\Leftarrow$. First, we claim that $\nu\left(\overline{I_{r}}, C\right)=$ $\nu\left({\overline{I_{r}}}^{(0)}, C^{(0)}\right)=b_{r}$. We always have $\nu\left(\overline{I_{r}}, C\right) \leq \nu\left({\overline{I_{r}}}^{(0)}, C^{(0)}\right)=b_{r}$. To check that the sign $<$ cannot hold, we may assume $C$ is irreducible, with generic point $y$, and we work in the ring $R$, the completion of $\mathcal{O}_{W, y}$ with respect to $\left(a_{1}, \ldots, a_{m}\right)$, an $A$-regular system of parameters which generates the ideal $I(C)_{y}$. We know $R=A^{\prime}\left[\left[a_{1}, \ldots, a_{r}\right]\right]$ (a power series ring), where $A^{\prime}$ is an appropriate local $A$-algebra. By (1), we may find in $\overline{I_{r}} R$ a series $h=M_{0}+\cdots+M_{b_{r}}+\cdots$ (sum of homogeneous parts in the power series ring), where $M_{b_{r}}$ is not a zero-divisor of $R$. Were, by contradiction, $\nu\left(\overline{I_{r}}, C\right)<b_{r}$, then we may find in $\overline{I_{r}}$ a series $g, g=M_{q}^{\prime}+\cdots$ (sum of homogeneous parts) where $M_{q}^{\prime} \neq 0$ and $q<b_{r}$. But then the series $g\left(h^{b_{r}-1}\right) \in \bar{I}_{r}{ }^{b-b_{r}} \subset J_{r}$ and (because $M_{b_{r}}$ is not a zero-divisor) has order $<b_{r}\left(b-b_{r}\right)$, a contradiction. So, the equality holds. But $B_{r}^{\prime}$ induces over the special fiber the object $B_{r}{ }^{(0)^{\prime}}$, in particular $C \subset \operatorname{Sing}\left(B_{r}\right):=\operatorname{Sing}\left(B_{r}^{(0)}\right.$. In [11] it is proved 
that $\nu\left(I_{0}^{(0)}, C^{(0)}\right) \geq b_{r}$. Thus, the implication $\Leftarrow$ is proved. It follows $C$ is a $B_{r}$ center.

(c) By (b), such a center $C$ is also a $\omega$-center. Then, in case $(\alpha)$ the equality follows from the definition of $\omega$-center. In case $(\beta)$, from this definition and Proposition 5.3. Note that in this case $a_{1}+\cdots+a_{m}=b-b_{r}$.

(d) The proof is a calculation entirely analogous to that for the case where $A=k$ is a field, and we shall omit it.

If (using the notation above) $B_{r}^{\prime}=\left(W_{r} \rightarrow S, J_{r}, b^{\prime}, E_{r}\right)$ is the object associated to an $A$-basic object $B_{r}=\left(W_{r} \rightarrow S, I_{r}, b, E_{r}\right)$, given a closed point $y \in \operatorname{Sing}\left(B_{r}^{\prime}\right)=\operatorname{Max}\left(\omega_{r}\right)(8.2)(\mathrm{b})$, we may find an element $f \in \Delta^{b^{\prime}-1}\left(J_{r} / S\right)_{y}$ defining, on a neighborhood of $y$, an $S$-hypersurface $Z$ satisfying condition (A1) of 6.2. But in general (A2) of 6.2 won't be satisfied, hence $Z$ won't be adapted to $B_{r}^{\prime}$. To overcome this difficulty we introduce another $A$-basic object, denoted by $B_{r}^{\prime \prime}$ (see $\left.[11,9.5]\right)$. This object $B_{r}^{\prime \prime}$ will be essential in inductive arguments.

8.3. Here we use the notation of 5.1. Consider a $t$-permissible sequence of $A$-basic objects

$$
\text { (1) } B_{0} \leftarrow \cdots \leftarrow B_{r}
$$

where we write $B_{j}=\left(W_{j} \rightarrow S, I_{j}, b, E_{j}\right)$.

(a) We shall say that an open set $U \subseteq W_{r}$ is amenable for $B_{r}$ if the following property holds. As usual, we write $t_{r}=\left(\omega_{r}, n_{r}\right)$. Let $\max \left(t_{r \mid U}\right)=$ $(\bar{\omega}, \bar{n})$ (where $\bar{\omega}=\max \left(\omega_{r \mid U}\right)$ ). Then, we require that for hypersurfaces $H_{1}^{*}, \ldots, H_{\bar{n}}^{*}$ in $E_{r}^{-}$we have:

$$
\operatorname{Max}\left(t_{r \mid U}\right)=\operatorname{Max}\left(\omega_{r \mid U}\right) \cap H_{1}^{*} \cap \cdots H_{\bar{n}}^{*} \cap U
$$

These hypersurfaces are uniquely determined, by the maximality of $\bar{n}$. Note that this concept depends not just on $B_{r}$ but also on its position in a $t$ permissible sequence like sequence (1) above.

If we may take $U=W_{r}$ as our amenable open, we say that $B_{r}$ is amenable.

(b) Note that if, in (1), $x \in \operatorname{Sing}\left(B_{r}\right)$, by the upper-semicontinuity of $t_{r}$ and the definitions we may find an open neighborhood $U$ of $x$ in $W_{r}$ such that $t_{r}(x)$ is the maximum of $t_{r \mid U}$ and $U$ is amenable for $B_{r}$.

(c) The object $B_{r}{ }^{\prime \prime}$. Let $U$ be a amenable open for $B_{r}$. Then we define a new $A$-basic object $\left(B_{r \mid U}\right)^{\prime \prime}$ as follows. Let $B_{r}^{\prime}=\left(W_{r}, J_{r}, b^{\prime}, E_{r}\right)$. Then, $\left(B_{r \mid U}\right)^{\prime \prime}=\left(U, I_{r}^{\prime \prime}, b^{\prime}, E_{r}^{+}\right)$, where

$$
I_{r}^{\prime \prime}=\left(J_{r}+I\left(H_{1}^{*}\right)^{b^{\prime}}+\cdots+I\left(H_{\bar{n}}^{*}\right)^{b^{\prime}}\right)_{\mid U}, \quad E_{r}^{+}=\left(E_{r} \backslash E_{r}^{-}\right)_{\mid U}
$$

(concerning $E_{r}^{-}$, see 5.1). By (b), given a point $x \in \operatorname{Sing}\left(B_{r}\right)$ always there is a neighborhood $U$ os $x$ such that $\left(B_{r \mid U}\right)^{\prime \prime}$ is defined.

When $B_{r}$ is amenable and we take $U=u s\left(B_{r}\right)$ we simply write $B_{r}^{\prime \prime}$. 
Proposition 8.4. Let $B_{0} \leftarrow \cdots \leftarrow B_{r}$ be a t-permissible sequence of $A$ basic objects, $B_{j}=\left(W_{j} \rightarrow S, I_{j}, b, E_{j}\right)$, where $B_{r}$ is amenable, and $B_{r}^{\prime \prime}$ the associated object just introduced. Then we have:

(a) $\left(B_{r}^{\prime \prime}\right)^{(0)}=\left(B_{r}^{(0)}\right)^{\prime \prime}$

(b) A center $C$ for $B_{r}$ is t-permissible if and only if it is $B_{r}^{\prime \prime}$-permissible.

(c) For every irreducible $B_{r}^{\prime \prime}$-center $C, \nu\left(I_{r}^{\prime \prime}, C\right)=\nu\left(I_{r}^{\prime \prime(0)}, C^{(0)}\right)=b^{\prime}$ (the index of $\left.B_{r}^{\prime \prime}\right)$.

(d) Let $C \subset W_{r}$ be a $B_{r}$-center which is t-permissible (or $B_{r}^{\prime \prime}$-permissible, it is the same.) Consider the transformations $B_{r}^{\prime \prime} \leftarrow\left(B_{r}^{\prime \prime}\right)_{1}$ and $B_{r} \leftarrow B_{r+1}$ with center $C$, and the object $B_{r+1}^{\prime \prime}$ associated to $B_{r+1}$. Assume $\max \left(t_{r}\right)=$ $\max \left(t_{r+1}\right)$. Then, $\left(B_{r}^{\prime \prime}\right)_{1}=\left(B_{r+1}\right)^{\prime \prime}$

(e) $B_{r}^{\prime \prime}$ is a locally nice object.

Proof. (a) easily follows from the definitions. (b) follows from the definition of $I_{r}^{\prime \prime}$, using 8.2(b) and the fact that $\operatorname{Max}\left(t_{r}\right)=\operatorname{Max}\left(\omega_{r}\right) \cap H_{1}^{*} \cap \cdots H_{\bar{n}}^{*}$ (recall that here $U=W_{r}$ ). (c) follows from (c) of 8.2 and the fact that such a center $C$ is contained in each hypersurface $H_{i}^{*}$.

Concerning (d), let $B_{r+1}^{\prime \prime}=\left(W_{r+1}, I_{r+1}^{\prime \prime}, b^{\prime \prime}, E_{r}^{+}\right)$. We must prove that, via the morphism $p: W_{r+1} \rightarrow W_{r}$ (the blowing up with center a $t$-permissible center $C$, with exceptional divisor $H), I_{r+1}^{\prime \prime}$ coincides with the controlled transform $I(H)^{-b^{\prime \prime}} I_{r}^{\prime \prime} \mathcal{O}_{W_{r+1}}=L$. Let $H_{j}^{\prime}$ denote the strict transform of $H_{j}^{*}$ via $p$, note that $H \in E_{r+1}^{+}$(because $\left.\max \left(t_{r}\right)=\max \left(t_{r+1}\right)\right)$. Letting $\mathcal{O}^{\prime}:=$ $\mathcal{O}_{W_{r+1}}$, we have:

$$
\begin{aligned}
L & =I(H)^{-b^{\prime}} I_{r}^{\prime \prime} \mathcal{O}^{\prime}=I(H)^{-b^{\prime}}\left[I_{r}^{\prime}+I\left(H_{1}^{*}\right)^{b^{\prime}}+\cdots+I\left(H_{N}^{*}\right)^{b^{\prime}}\right] \mathcal{O}^{\prime} \\
& =I(H)^{-b^{\prime}} I_{r}^{\prime}+\left(I(H)^{-1} I\left(H_{1}^{*}\right)\right)^{b^{\prime}}+\cdots+\left(I(H)^{-1} I\left(H_{N}{ }^{*}\right)^{b^{\prime}}\right] \mathcal{O}^{\prime} \\
& =I_{r+1}{ }^{\prime}+I\left(H_{1}^{\prime}\right)^{b^{\prime}}+\cdots+I\left(H_{N}^{\prime}\right)^{b^{\prime}}
\end{aligned}
$$

and the latter is precisely $I_{r+1}^{\prime \prime}$, as desired.

(e) Consider the smallest index $s$ such that $\max \left(t_{s}\right)=\cdots=\max \left(t_{r}\right)$. As usual, $t_{j}$ denotes the $j$-th $t$-function of the sequence of special fibers. Working with $B_{s}^{\prime \prime}$, by $8.2(\mathrm{c})$ and 6.3 , if $x \in \operatorname{Sing}\left(B_{s}^{\prime \prime}\right)$ is a closed point we may get an open neighborhood $V$ of $x$ in $W_{s}$ and an element $h \in \Gamma\left(V, \Delta^{b^{\prime \prime}-1}\left(I_{s}^{\prime \prime} / S\right)\right)$ defining an $A$-hypersurface on $V$. This is an adapted hypersurface (for $B_{r}^{\prime \prime}$ restricted to $V$ ). Indeed, clearly (A1) of 6.2 is satisfied, and (A2) is obvious because $E_{s}^{*}$ is empty. Letting $x$ vary in the (dense) set of closed points of $\operatorname{Sing}\left(B_{s}^{\prime \prime}\right)$ we get an open covering $\left\{V_{j}\right\}, j=1, \ldots, q$ of $\operatorname{Sing}\left(B_{s}^{\prime \prime}\right)$ and hypersurfaces $Z_{j}$ defined on $V_{j}$ and adapted for the restriction of $B_{s}^{\prime \prime}$ to $V_{j}$, for all $j$. Transforming with center $C_{s}$, letting $V_{1 j}$ be the pre-image of $V_{j}$ in $W_{s+1}$ and $Z_{1 j}$ the strict transform of $Z_{j}$, by Giraud's Lemma $6.10, Z_{1 j}$ is 
locally defined by an element of $\Delta^{b^{\prime \prime}-1}\left(I_{s+1}^{\prime \prime} / S\right)$ and is transversal to $E_{s+1}{ }^{+}$ (which consists of the exceptional divisor $H_{1}$ of the transformation only), because $C_{s} \cap V_{j}$ is contained in $Z_{j}$. Thus, $Z_{1 j}$ is adapted to the restriction of $B_{s+1}^{\prime \prime}$ to $V_{1 j}$, for all $j$. Reiterating, we get a covering of $\operatorname{Sing}\left(B_{r}^{\prime \prime}\right)$ by open sets $V_{(r-s), j}, j=1, \ldots, q$ of $W_{r}$ and on each $V_{(r-s), j}$ an $A$-hypersurface $Z_{(r-s), j}$, adapted to the restriction of $B_{r}^{\prime \prime}$ to $V_{(r-s), j}$, for all $j$. Thus, $B_{r}^{\prime \prime}$ is locally nice, as claimed.

8.5. More on the resolution algorithm over a field. Now we check the assertions made in 2.10 , by using the theory of Section 7 and the present one (in case $A=k$, a field).

We take as the open set $U$ of 2.10 an amenable neighborhood of $w$. Then the nice object $B_{s}^{\prime \prime}$ of 8.3 is defined on $U$, hence its associated homogenized object $\mathcal{H}\left(B_{s}^{\prime \prime}\right):=\left(H B_{s}^{\prime \prime}\right)$ (which is again nice) admits an adapted hypersurface $Z_{s U}$ (or simply $Z_{s}$ ) containing $w$, defined on $U$. From the assumption "dim $\left(\operatorname{Max}\left(t_{s}\right)<d-1 "\right.$ made in 2.8, $Z_{s}$ is inductive. This is the hypersurface $Z_{s}$ of 2.8 , while our object $B_{s}^{*}$ is $\left(H B_{s}^{\prime \prime}\right)_{Z_{s}}(6.11(\mathrm{~b}))$. (More properly, we should write, e.g., $B_{s, U}^{*}=\mathcal{H}\left(\left(B_{s \mid U}\right)^{\prime \prime}\right)_{Z_{s U}}$ rather than $B_{s}^{*}$, we try to simplify the notation).

Now we check properties $(i)-(i v)$ of 2.8 .

(i) By 6.13, $\operatorname{Sing}\left(B_{s}^{*}\right)=\operatorname{Sing}\left(H B_{s}{ }^{\prime \prime}\right)$. Since, by Proposition 7.4, $B_{s}{ }^{\prime \prime}$ and $H B_{s}{ }^{\prime \prime}$ are W-equivalent, we obtain $\operatorname{Sing}\left(H B_{s}{ }^{\prime \prime}\right)=\operatorname{Sing}\left(B_{s}{ }^{\prime \prime}\right)$. Combining these facts, we get $(i)$ of 2.8 .

(ii) By repeated application of $6.13(\mathrm{i})$, (ii), from the algorithmic resolution (2) of 2.8 (obtained by the inductive hypothesis) we get a permissible sequence

$$
\text { (1) } \quad\left(H B_{s}^{\prime \prime}\right) \leftarrow\left(H B_{s}^{\prime \prime}\right)_{1} \leftarrow \cdots
$$

By the W-equivalence of $\left(H B_{s}{ }^{\prime \prime}\right)$ and $B_{s}{ }^{\prime \prime}(7.4)$, the sequence (1) induces (by using the same centers) a permissible sequence

$$
B_{s}^{\prime \prime} \leftarrow\left(B_{s}^{\prime \prime}\right)_{1} \leftarrow \cdots
$$

which, by 8.4 (b), induces (by using again the same centers) a $t$-permissible sequence

$$
\widetilde{B}_{s} \leftarrow \widetilde{B}_{s 1} \leftarrow \cdots
$$

as claimed in (ii) of 2.8 .

(iii) By 6.10, us $\left(B_{s+j}^{*}\right)$ may be identified to the strict transform $Z_{j}$ of $Z_{s}$ to the scheme $u s\left(\left(H B_{s}^{\prime \prime}\right)_{j}\right)$. By the W-equivalence of $B_{s}{ }^{\prime \prime}$ and $\left(H B_{s}{ }^{\prime \prime}\right)$, $u s\left(\left(H B_{s}^{\prime \prime}\right)_{j}\right)=u s\left(\left(B_{s}^{\prime \prime}\right)_{j}\right)$. But, from our assumption "max $\left(\widetilde{t}_{s}\right)=\cdots=$ $\max \left(\widetilde{t}_{s+q}\right)$ ", we get $\left(B_{s}^{\prime \prime}\right)_{j}=\left(\widetilde{B}_{r+j}\right)$. Since $Z_{j}=u s\left(B_{s+j}^{*}\right)$, the fact that $u s\left(B_{s+j}^{*}\right)=\operatorname{Max}\left(\widetilde{t}_{s+j}\right)$ follows from 8.4. 
(iv) Since $\operatorname{Sing}\left(B_{s+j}^{*}\right)$ can be identified to $\operatorname{Max}\left(\widetilde{t}_{s+j}\right) \subseteq \operatorname{Sing}\left(B_{s+j}\right), \widetilde{g}_{j}$ defines a function as claimed. The fact that its value $\widetilde{g}_{j}(x)$ is well-defined comes from the following observation, by restricting to a suitable amenable open neighborhood of $x$ and using the compatibility of our constructions with etale pull-backs, in particular open inclusions.

Let $B=(U \rightarrow S, I, b, E)$ be a nice basic object, $(H B)$ its associated homogenized (nice) object, $Z, Z^{\prime}$ adapted hypersurfaces for $(H B), B^{*}=$ $(H B)_{Z}, B^{\prime *}=(H B)_{Z^{\prime}}^{\prime}$. Consider the resolution functions $g_{0}, \ldots, g_{m}$ of $B^{*}$ and $h_{0}, \ldots, h_{m^{\prime}}$ of $B^{\prime *}$ respectively (known by induction on the dimension). We claim that $g_{i}=h_{i}$, for all $i$. This means that $m=m^{\prime}$, for all $i$ both $g_{i}$ and $h_{i}$ have the same domain, and they take equal values.

Indeed, for $j=0$, note that, by [11], $S_{0}:=\operatorname{Sing}\left(B^{*}\right)=\operatorname{Sing}\left((H B)_{Z}\right)=$ $\operatorname{Sing}((H B))=\operatorname{Sing}\left((H B)_{Z^{\prime}}\right)=\operatorname{Sing}\left(B^{\prime *}\right)$ hence the domains of $g_{0}$ and $h_{0}$ agree. If $x \in S_{0}$, by 7.13 we get etale neighborhoods $p:(Y, y) \rightarrow(Z, x)$ and $p^{\prime}:(Y, y) \rightarrow\left(Z^{\prime}, x\right)$ such that $p^{*}\left(B^{*}\right)=p^{\prime *}\left(B^{\prime *}\right):=\bar{B}$. By the compatibility of the algorithm with etale morphisms, $p^{*}\left(g_{0}\right)$ and $p^{* *}\left(h_{0}\right)$ agree on $\operatorname{Sing}(\bar{B})$. Hence, $\widetilde{g_{0}}(x)=p^{*}\left(\widetilde{g_{0}}\right)(y)=p^{\prime *}\left(\widetilde{h_{0}}\right)(y)=\widetilde{h_{0}}(x)$. Consequently, $\operatorname{Max}\left(g_{0}\right)=$ $\operatorname{Max}\left(h_{0}\right)$ (say, $=C$ ). But $C$ is the first center we use in the resolution both for $B^{*}$ and $B^{\prime *}$. By [11], $C$ is also a permissible center for $(H B)$ (or its Wequivalent object $B$ ). Transform these objects with center $C$, getting $B^{*} \longleftarrow$ $B^{*}{ }_{1}, B^{\prime *} \leftarrow B^{\prime *}{ }_{1}, H B \leftarrow H B_{1}, B \leftarrow B_{1}$ respectively. We know that us $\left(B^{*}{ }_{1}\right)$ (resp. us $\left(B^{\prime *}{ }_{1}\right)$ ) is the strict transform $Z_{1}$ (resp. $Z_{1}^{\prime}$ ) of $Z$ (resp. $Z^{\prime}$ ) to $U_{1}:=$ $u s\left((H B)_{1}\right)=u s\left(B_{1}\right)$ (use 6.10). Again $S_{1}=\operatorname{Sing}\left(B^{*}{ }_{1}\right)=\operatorname{Sing}\left(\left(H B_{1}\right)\right)=$ $\operatorname{Sing}\left(B^{\prime *}{ }_{1}\right)\left(\right.$ both $\left.=\operatorname{Sing}\left(B_{1}\right)\right)$. Using again 7.13 , given an arbitrary point $x_{1}$ in $S_{1}$, lying over $x_{0} \in S \subset U$, we get commutative cartesian diagrams

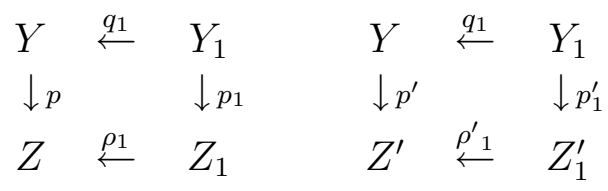

(where, by the compatibility of the resolution algorithm with etale pullbacks, $q_{1}$ is the first transformation in the algorithmic resolution process for $\bar{B})$ such that $p_{1}{ }^{*}\left(B^{*}{ }_{1}\right)=p_{1}{ }^{*}\left(B^{\prime *}{ }_{1}\right):=\bar{B}_{1}$. Then, both $p_{1}{ }^{*}\left(g_{0}\right)$ and $p_{1}^{\prime *}\left(h_{1}\right)$ must be the first resolution function for $\bar{B}$, hence they must agree. Exactly as in case $j=0$ we conclude that $g_{1}\left(x_{1}\right)=h_{1}\left(x_{1}\right)$. Since $x_{1}$ was arbitrary in $S, g_{1}=h_{1}$. We may iterate this procedure, each time using 7.13 to show that $m=m^{\prime}$ and $g_{i}=h_{i}$ for all $i$.

We should check that the functions $g_{j}$ thus defined are compatible with etale pull-backs (we used this fact in the inductive step). But this is simple, and we leave it to the reader (or see [5] or [11]). 


\section{Algorithmic equiresolution}

9.1. In this section we work with the VW-resolution algorithm (for basic objects over fields of characteristic zero) discussed in 2.8, 2.10 and 8.5. Henceforth this will be referred to as the algorithm. As usually, $\mathcal{A}$ is the class of rings of 3.1 .

We shall prove Theorem 1.1. To that effect, given a ring $A \in \mathcal{A}$ and an $A$-basic object $B=(W \rightarrow S, I, b, E), S=\operatorname{Spec}(A)$, we shall introduce certain conditions $\mathcal{E}_{j}, j=0,1, \ldots$, which may be valid or not. Intuitively, these conditions work as follows. Let

$$
B^{(0)}:=B_{0}^{(0)} \leftarrow \cdots \leftarrow B_{r}^{(0)}
$$

be the algorithmic resolution of the fiber $B^{(0)}$ of $B$, obtained by using algorithmic resolution functions $g_{i}, i=0, \ldots, r-1$, which yield resolution centers $C_{i}=\operatorname{Max}\left(g_{i}\right) \subset u s\left(B_{i}^{(0)}\right)$. For $0 \leq j<r$ the validity of $\mathcal{E}_{j}$ means that all the operations involved to complete the first $j-1$ steps in the algorithmic resolution (1) (i.e., what is necessary to obtain the $j$-truncation of (1)) can be extended, in a natural way, along $S=\operatorname{Spec} A$. Theorem 1.1 will be an immediate consequence of this theory. Let us discuss these conditions more carefully.

We define conditions $\mathcal{E}_{j}$ for every non-negative integer. We declare them vacuously valid for $j \geq r$. In particular if, in (1), $r=0$, i.e., $\operatorname{Sing}\left(B^{(0)}\right)=\emptyset$, then $B$ is algorithmically equisolvable. So, assume $r>0$. Then, for values $j=0, \ldots, r-1$, condition $\mathcal{E}_{j}$, if valid, defines centers $C_{i}, i=0, \ldots, j$, where more precisely $C_{0}$ is a center for $B=B_{0}, C_{1}$ is a center for $B_{1}=$ $\mathcal{T}\left(B_{0}, C_{0}\right), \ldots, C_{j}$ is a center for $B_{j}=\mathfrak{T}\left(B_{j-1}, C_{j-1}\right)$. Thus, if $\mathcal{E}_{j-1}$ is valid, $1 \leq j \leq r$, one has an induced permissible sequence of $A$-basic objects

$$
(2)_{j} \quad B_{0} \leftarrow \cdots \leftarrow B_{j}
$$

These conditions $\mathcal{E}_{j}$ will satisfy the following properties:

(a) The sequence $(2)_{j}$ induces, by taking fiber, the $j$-truncation of the algorithmic resolution of $B^{(0)}$.

(b) If $i<j$, then the sequence $(2)_{i}$ is the $i$-truncation of the analogous sequence $(2)_{j}$.

(c) If $f: W^{\prime} \rightarrow W$ is an etale morphism and $B^{\prime}$ is the $A$-basic object induced from $B$ by pull-back, if condition $\mathcal{E}_{j}$ is valid for $B$ then it is also valid for $B^{\prime}$ and the centers $C_{j}^{\prime}$ associated to $B^{\prime}$ are the pull-backs of those associated by $\mathcal{E}_{j}$ to $B$.

(d) The conclusion of (c) remains true if $W^{\prime}=W \times{ }_{S} S^{\prime}$, where $S^{\prime} \rightarrow S$ is induced by a ring homomorphism (of rings in $\mathcal{A}$ ) $A \rightarrow A^{\prime}$ and $f: W^{\prime} \rightarrow W$ is the first projection (i.e., $\mathcal{E}_{j}$ is stable under change of the base ring $A$ ). 
These conditions, satisfying the mentioned properties, will be defined inductively on $d=\operatorname{dim} B$. This will be done in the following sub-sections. But, accepting the results just stated, we may prove Theorem 1.1 as follows.

9.2. Proof of Theorem 1.1. Given an $A$-basic object $B$, with fiber $B^{(0)}$, let condition $\mathcal{E}_{i}$ be valid for $i=0, \ldots, s-1$. Then define $e(B):=s$. There is an associated $A$-permissible sequence $B=B_{0} \leftarrow \cdots \leftarrow B_{s}$ (this is $(2)_{j}$ of 9.1, with $j=s$ ), using centers $C_{i} \subset u s\left(B_{i}\right), i=0, \ldots, s-1$. These are the centers we associate to $B$ to complete the definition of the function of Theorem 1.1. The claimed properties of this function follow from properties (a), (b), (c) and $(\mathrm{d})$ of conditions $\mathcal{E}_{i}$ stated in 9.1.

Definition 9.3. Given an $A$-basic object $B$ as in 9.1 we say that it is algorithmically equisolvable if conditions $\mathcal{E}_{j}$ are valid for every $j \geq 0$. Equivalently, since conditions $\mathcal{E}_{j}$ are vacuously valid for $j \geq r$, we may require that $\mathcal{E}_{j}$ be valid for $j=0, \ldots, r-1$ (with $r$ as in sequence (1) in 9.1) or that in Theorem 1.1 it must be $e(B)=\ell\left(B^{(0)}\right)$.

In this case, the sequence $(2)_{r}$ of 9.1 that is determined will be called the algorithmic equiresolution sequence of $B$.

Of course, an $A$-basic object may not be algorithmically equisolvable. For instance, the basic object of Example 4.3 has non-empty singular locus but it does not admit any permissible center. Hence it cannot have any equiresolution.

Now let us precisely define conditions $\mathcal{E}_{i}$.

9.4. Defining $\mathcal{E}_{j}$ when $\operatorname{dim}(B)=1$. Let $(1)$ of 9.1 be the algorithmic resolution of the special fiber $B^{(0)}$. We shall define, for $0 \leq j<r$, conditions $\mathcal{E}_{j}$, in such a way that if $\mathcal{E}_{j}$ is valid, then the resulting sequence $(2)_{j}$ (of 9.1) is $\rho$-permissible (see 5.7).

Start with $\mathcal{E}_{0}$. Consider $B:=B_{0}$. Notice that necessarily $\max \left(\omega_{0}\right)>0$. In this case, the zeroth algorithmic center $C^{(0)}$ of the fiber $B^{(0)}$ is $\operatorname{Max}\left(t_{0}\right)$. To define $\mathcal{E}_{0}$, take an open cover $\left\{U_{i}\right\}$ of $\operatorname{Max}\left(t_{0}\right)$ such that each $U_{i}$ is amenable for $B$, hence $\left(B_{\mid U_{i}}\right)^{\prime \prime}:=B_{i}^{\prime \prime}=\left(U_{i} \rightarrow S, I_{i}^{\prime \prime}, b^{\prime \prime}, E_{i}^{\prime \prime}\right)$ is defined (see 8.3). Then, to have $\mathcal{E}_{0}$ valid, we require that, for all $i, \Delta^{b^{\prime \prime}-1}\left(I_{i}^{\prime \prime} / S\right)$ define a $B_{i}^{\prime \prime}$-permissible center $C_{i}$. Since the locally defined objects $B_{i}^{\prime \prime}$ agree on intersections (this follows from the construction in 8.3), this requirement is independent of the choice of the cover and the different $C_{i}$ glue together to yield a well-defined center $C$ which is $B$-permissible (because, by 8.4 , $C$ restricted to each $U_{i}$ is $B_{\mid U_{i}}$ permissible). This $C$ is the center $\mathcal{E}_{0}$ defines. Property (a) is true because because $\left(B_{\mid U_{i}}\right)^{\prime \prime}$ restricted to the fiber is $\left(B^{(0)}{ }_{\mid U_{i}}\right)^{\prime \prime}$ and $\operatorname{Sing}\left(B^{(0)}{ }_{\mid U_{i}}\right)^{\prime \prime}=\mathcal{V}\left(\Delta^{b-1}\left(I^{(0)}{ }_{\mid U_{i}}\right)\right)=\operatorname{Max}\left(t_{0}\right) \cap U_{i}$. Property (b) is clear. Moreover, $C$ is $t$-permissible $(8.4(\mathrm{~b}))$. 
Now, if $j>1$, assuming $\mathcal{E}_{s}$ defined for $s<j$, we introduce condition $\mathcal{E}_{j}$. Looking at $B_{j}^{(0)}$, there are two cases: $(\alpha) \max \left(\omega_{j}\right)>0,(\beta) \max \left(\omega_{j}\right)=0$.

Consider case $(\alpha)$. To declare $\mathcal{E}_{j}$ valid, first we require that conditions $\mathcal{E}_{s}$ be valid, for $s<j$. Hence, we have a $t$-permissible sequence $B_{0} \leftarrow \cdots \leftarrow B_{j}$. Recall that in this case the $j$-th center $C_{j}^{(0)}$ in (1) of 9.1 is $\operatorname{Max}\left(t_{j}\right)$. Apply to $B_{j}$ the technique used in the case $j=0$. Namely, cover $\operatorname{Max}\left(t_{j}\right)$ by amenable open sets, so that nice basic $A$-objects $\left(B_{j}^{\prime \prime}\right)_{i}=\left(U_{i} \rightarrow S, I_{i}^{\prime \prime}, b_{i}^{\prime \prime}, E_{i}^{\prime \prime}\right)$ are defined (see 8.3). To finish the definition of $\mathcal{E}_{j}$ in this case we require that the subscheme defined by $\Delta^{b_{i}^{\prime \prime}-1}\left(I_{i}^{\prime \prime} / S\right)$ be a $\left(B_{j}^{\prime \prime}\right)_{i}$-center $C_{j i}$, for all $i$. As above, this is independent of the chosen cover, and these centers patch together to produce a $t$-permissible center $C_{j}$ for $B_{j}$, which will be the $B_{j}$ center determined by $\mathcal{E}_{j}$. Requirement (a) is treated as above (case $j=0$ ) and (b) is clear.

Now consider case $(\beta)$. Here, the object $B_{j}$ is premonomial. To have condition $\mathcal{E}_{j}$ satisfied we require that $B_{j}$ be monomial, and we take as the associated center the canonical monomial center. The verification of properties (a) though (d) is simple.

9.5. It remains to check, in case $(\alpha)$, properties (c) and (d). We are not going to present the details of this verification, either here or in the other parts of the discussion of conditions $\mathcal{E}_{j}$. In fact, the verification is either immediate or a consequence of calculations in certain local rings (see, e.g., 3.9 and 3.10). These calculations are simple once we use the following observations.

For (c), use the identification of Proposition 7.6. Concerning (d), note that if $B=(W \rightarrow \operatorname{Spec} A, I, b, E)$ is an $A$-basic object, $w \in W$ a closed point, $A \rightarrow A^{\prime}$ a homomorphism in $\mathcal{A}, W^{\prime}=W \times_{A} A^{\prime} \rightarrow W$ the first projection, $w^{\prime} \in W^{\prime}$ a point lying over $w, R=\mathcal{O}_{W, w} \stackrel{\phi}{\rightarrow} \mathcal{O}_{W^{\prime}, w^{\prime}}^{\prime}=R^{\prime}$ the induced homomorphism, then: (a) an $A$-regular system of parameters $\mathbf{a}=\left(a_{1}, \ldots, a_{n}\right)$ of $R$ maps into an $A^{\prime}$-regular system of parameters $\mathbf{a}^{\prime}=$ $\left(a_{1}^{\prime}, \ldots, a_{n}^{\prime}\right)$ of $R^{\prime}$, (b) if $\widehat{R}$ (resp. $\widehat{R}^{\prime}$ ) is the completion of $R$ with respect to a (resp. of $R^{\prime}$ with respect to $\mathbf{a}^{\prime}$ ) then there is an identification of $\widehat{R}$ and the power series ring $A_{1}\left[\left[x_{1}, \ldots, x_{n}\right]\right]$ (resp. $\widehat{R}=A_{1}^{\prime}\left[\left[x_{1}, \ldots, x_{n}\right]\right]$ ) where $A_{1}=R /\left(a_{1}, \ldots, a_{n}\right)$ (resp. $\left.A_{1}^{\prime}=R^{\prime} /\left(a_{1}^{\prime}, \ldots, a_{n}^{\prime}\right)\right)$, with these, $\phi$ induces the natural homomorphism from $A_{1}=R /\left(a_{1}, \ldots, a_{n}\right)$ to $A_{1}^{\prime}=R^{\prime} /\left(a_{1}^{\prime}, \ldots, a_{n}^{\prime}\right)$ (sending each $x_{i}$ to itself); we also use the corresponding known result in case $A$ and $A^{\prime}$ are fields (2.12).

In the discussion of the case where the dimension $d$ of $B$ is arbitrary we shall need a lemma that we state next. Its proof will be presented in 9.10. Informally, it says that if $(H B)$ is the homogenized $A$-object associated to $B$ (nice), $Z$ and $Z^{\prime}$ are inductive hypersurfaces for $(H B)$ and we assume, inductively on the dimension, that the inductive objects $(H B)_{Z}$ and $(H B)_{Z^{\prime}}$ are 
algorithmically equisolvable, then the 0 -th centers $C_{0}$ and $C_{0}^{\prime}$ of their algorithmic equiresolutions coincide: $C_{0}=C_{0}^{\prime}$. Moreover, under suitable strong permissibility assumptions similar equalities hold for the other algorithmic resolution centers associated to $(H B)_{Z}$ and $(H B)_{Z^{\prime}}$.

Lemma 9.6. In the previous notation, assume conditions $\mathcal{E}_{j}$, satisfying $(a),(b),(c)$ of 9.1 , are defined for every possible $j$ when the dimension of our basic object is $<d$. Let $B=(W \rightarrow S, I, b, E)$ be a nice d-dimensional basic object over $A,(H B):=\mathcal{H}(B)=(W \rightarrow S, \mathcal{H}(I / S, b), b, E)(c f .7 .2$, it is again nice), $Z$ and $Z^{\prime}$ inductive hypersurfaces for $(H B)$. Let $(H B)_{Z}$ and $(H B)_{Z^{\prime}}$ be the corresponding inductive objects. Assume that both $(H B)_{Z}$ and $(H B)_{Z^{\prime}}$ are algorithmically equisolvable (i.e., they satisfy conditions $\mathcal{E}_{j}$ for all possible $j$, since their dimensions are equal to $d-1$, by our hypothesis these conditions are defined). Consider the corresponding algorithmic equiresolution sequences

$$
\begin{aligned}
& (H B)_{Z}=\left((H B)_{Z}\right)_{0} \leftarrow \cdots \leftarrow\left((H B)_{Z}\right)_{t} \\
& (H B)_{Z^{\prime}}=\left((H B)_{Z^{\prime}}\right)_{0} \leftarrow \cdots \leftarrow\left((H B)_{Z^{\prime}}\right)_{t}
\end{aligned}
$$

(Note that the length of these sequences, which depends on the special fibers only, must be the same, by the results of 8.5). Assume that both (1) and (2) are strongly permissible sequences (see 6.17), determined by centers $C_{i} \subset$ $u s\left(\left((H B)_{Z}\right)_{i}\right), C_{i}^{\prime} \subset u s\left(\left((H B)_{Z^{\prime}}\right)_{i}\right), i=0, \ldots, t-1$ respectively. Let

$$
\begin{aligned}
& (H B)_{0} \leftarrow(H B)_{1} \leftarrow \cdots \leftarrow(H B)_{t}, \\
& (H B)_{0}=(H B)_{0}^{\prime} \leftarrow(H B)_{1}^{\prime} \leftarrow \cdots \leftarrow(H B)_{t}^{\prime}
\end{aligned}
$$

be the induced permissible sequences (see 6.17). By 6.10, if $Z_{i}$ and $Z_{i}^{\prime}$ are the strict transforms of $Z$ and $Z^{\prime}$ to $(H B)_{i}$ and $(H B)_{i}^{\prime}$ respectively, there are identifications $Z_{i}=u s\left((H B)_{Z}\right)_{i}, Z_{i}^{\prime}=u s\left((H B)_{Z}^{\prime}\right)_{i}$.

Then, using these identifications, $C_{0}=C_{0}^{\prime}$, hence us $(H B)_{1}=u s\left((H B)_{1}^{\prime}\right)$; using this fact, $C_{1}=C_{1}^{\prime}$ (an equality of subschemes of us $\left((H B)_{1}\right)$, hence $\left.(H B)_{2}=(H B)_{2}^{\prime}\right)$, and so on, eventually getting $C_{\lambda}=C_{\lambda}^{\prime}$, (as subschemes of $\left.u s\left((H B)_{\lambda}\right)=u s\left((H B)_{\lambda}^{\prime}\right), \lambda=0, \ldots, t\right)$.

9.7. Defining conditions $\mathcal{E}_{j}$ when $\operatorname{dim} B=d$, an arbitrary positive integer. The case $d=1$ being explained, we shall proceed by induction on $d$. So, assuming the definition (having properties (a), (b), (c) and (d) of 9.1) known when the dimension of the $A$-basic object is less than $d$, we'll introduce conditions $\mathcal{E}_{j}$ for $B d$-dimensional. This will be done again by induction on $j$.

Start with $j=0$. Let $B=B_{0}$ be our $A$-basic object. Looking at the fiber $B^{(0)}$, its $\omega_{0}$ function must satisfy $\max \left(\omega_{0}\right)>0$. Consider $M=\operatorname{Max}\left(t_{0}\right)$. There are two cases: $\operatorname{dim} M=d-1$ or $\operatorname{dim} M<d-1$. 
(I) Case $\operatorname{dim} M=d-1$. Here, on the fiber $B^{(0)}$, the zeroth algorithmic resolution center is $M(1)$, the union of the components of $M$ of codimension one. We proceed as in the one-dimensional situation. Namely, consider locally defined nice $A$-basic objects $B_{i}^{\prime \prime}=\left(U_{i} \rightarrow S, I_{i}^{\prime \prime}, b_{i}^{\prime \prime}, E_{i}^{\prime \prime}\right)$ as in 8.3, where the open sets $U_{i}$ cover $M(1)$ and, for all $i, U_{i} \cap Y=\emptyset$, for any irreducible component $Y$ of codimension $>1$ of $M$. (This is possible, since by [11] $M(1)$ is regular.) Then we declare condition $\mathcal{E}_{0}$ valid if for each $i$ the $W$-ideal $\Delta^{b_{i}^{\prime \prime}-1}\left(I_{i}^{\prime \prime} / S\right)$ defines a permissible center $C_{i}$. Again, since the considered nice basic objects agree on each intersection $U_{i} \cap U_{i^{\prime}}$, the different $C_{i}$ define a $B$-center $C$. We take $C$ as the center that $\mathcal{E}_{0}$ associates to $B$. From the construction of the nice object $B_{i}^{\prime \prime}(8.3)$, this is independent of the choice of the open cover $\left\{U_{i}\right\}$. Conditions (a), (b), (c) and (d) of 9.1 are easily checked (see 9.4 and 9.5). This finishes the case where $j=0$ and $\operatorname{dim}(M)=d-1$.

(II) Case $\operatorname{dim} M<d-1$. In this situation, the zeroth algorithmic center $C_{0}^{(0)}$ in the resolution sequence (1) of 9.1 is defined inductively, on the dimension (using locally defined basic objects $B_{0}^{(0)}$ " , inductive hypersurfaces, homogeneization (7.2) and the corresponding inductive objects). Accordingly, we cover $M=\max \left(g_{0}\right)$ by amenable opens $U_{i}(i$ in a suitable set, $g_{0}$ is the 0 -th resolution function of $\left.B_{0}^{(0)}\right)$. Hence on each $U_{i}$ there is a nice $A$-basic object $B_{i}^{\prime \prime}=\left(U_{i} \rightarrow S, I_{i}^{\prime \prime}, b_{i}^{\prime \prime}, E_{i}^{\prime \prime}\right)$ admitting an adapted hypersurface $Z_{i} \subset U_{i}$ (see 8.3). For each $i$ take the homogenized object $\left(H B_{i}^{\prime \prime}\right):=\mathcal{H}\left(B_{i}^{\prime \prime}\right)=\left(U_{i} \rightarrow S, I_{i}^{*}, b_{i}^{\prime \prime}, E_{i}^{\prime \prime}\right)$ (where $\left.I_{i}^{*}:=\mathcal{H}\left(I_{i}^{\prime \prime} / S, b_{i}^{\prime \prime}\right)\right)$. The object $\left(H B_{i}^{\prime \prime}\right)$ is again nice, admitting $Z_{i}$ as an adapted hypersurface. From our assumption on $\operatorname{dim}(M)$, by 8.4 (b) and $6.12, Z_{i}$ also satisfies (A3) of 6.2 , i.e., $Z_{i}$ is inductive. Consider the inductive object $B_{i}^{*}:=\left(H B_{i}^{\prime \prime}\right)_{Z_{i}}=\left(Z_{i} \rightarrow\right.$ $\left.S, \mathrm{C}\left(I_{i}^{*} / S, Z_{i}\right), \tilde{b}_{i}, \tilde{E}_{i}\right)$, see 6.11 (a). By induction we know what it means the expression "condition $\mathcal{E}_{s}$ is valid for $B_{i}^{*}$, for every $i$ and $s$ ". So, we declare: $\mathcal{E}_{0}$ is valid for $B$ if the following conditions hold: (a) for all $i, \mathcal{E}_{s}$ is valid for $B_{i}^{*}$, for all $s \geq 0$; (b) the center $C_{i}$ that condition $\mathcal{E}_{0}$ is associates to $B_{i}^{*}$, is strongly permissible (see 6.17). Then we claim that these centers $C_{i}$ agree on intersections, defining a $B$-permissible center $C \subset W$. These statements follow from the following:

Observation: Let $V_{1}$ and $V_{2}$ be amenable open neighborhoods of $x \in M$, such that nice basic objects $B_{1}^{\prime \prime}$ and $B_{2}^{\prime \prime}$, with inductive hypersurfaces $Z_{1}$ and $Z_{2}$, are defined on $V_{1}$ and $V_{2}$ respectively, as in 8.3. Let $B_{i}^{*}:=\left(H B_{i}^{\prime \prime}\right)_{Z_{i}}$, $i=1,2$, both satisfying condition $\mathcal{E}_{s}$, for all $s \geq 0$. Let $U \subseteq V_{1} \cap V_{2}$ be an open neighborhood of $x, C_{i}$ the center that condition $\mathcal{E}_{0}$ assigns to $B_{i}^{*}, i=1,2$. Assume $C_{i}, i=1,2$, are strongly permissible centers. Then, $C_{1} \cap U=C_{2} \cap U$. 
To prove this observation note that, by the construction of $8.3, B_{1 \mid U}^{\prime \prime}=$ $B_{2 \mid U}^{\prime \prime}\left(\right.$ say $\left.=B^{\prime \prime}\right)$. Now, both $Z_{1} \cap U$ and $Z_{2} \cap U$ are hypersurfaces on $U$ adapted to $B^{\prime \prime}$. Then by applying Lemma 9.6 (case $\lambda=0$ ) we get $C_{1} \cap U=$ $C_{2} \cap U$, as needed.

9.8. Now, inductively, assuming conditions $\mathcal{E}_{s}$, satisfying properties (a), (b), (c) and (d) of 9.1, have been defined for $s<j, j>0$, let's introduce condition $\mathcal{E}_{j}$. By our inductive hypothesis (see $9.1,(2)_{j}$ ) we get a permissible sequence of $A$-basic objects

$$
\text { (1) } B=B_{0} \leftarrow \cdots \leftarrow B_{j}
$$

inducing on fibers a sequence

$$
B^{(0)}=B_{0}^{(0)} \leftarrow \cdots \leftarrow B_{j}^{(0)}
$$

(where (2) is the $j$-truncation of the algorithmic resolution of $B^{(0)}$ ). Looking at the functions $\omega_{p}$ corresponding to the sequence (2), we distinguish two cases: (I) $\max \left(\omega_{j}\right)=0$, (II) $\max \left(\omega_{j}\right)>0$. In case (I), $B_{j}$ is premonomial. We declare condition $\mathcal{E}_{j}$ valid if $B_{j}$ is monomial and we take the corresponding center $C_{j}$ to be the canonical center in this case (see 5.4.)

In situation (II), looking at the functions $t_{p}$ corresponding to the sequence (2), and letting $M:=\operatorname{Max}\left(t_{j}\right)$ and $d=\operatorname{dim}(B)$, we distinguish the following two possibilities: $(\alpha) \operatorname{dim} M<d-1,(\beta) \operatorname{dim} M=d-1$.

Consider $(\alpha)$ first. This is the situation where to define the $j$-th center $C_{0}^{(0)}$ in the algorithmic resolution of $B^{(0)}$ we use induction on the dimension (via local associated homogenized nice basic objects, inductive hypersurfaces, inductive objects). Take the index $q$ such that $\max \left(t_{q}\right)=$ $\max \left(t_{q+1}\right)=\cdots=\max \left(t_{j}\right)$ but $\max \left(t_{q-1}\right)>\max \left(t_{q}\right) . \operatorname{dim}\left(M_{q}\right)<d-1$, with $M_{q}:=\operatorname{Max}\left(t_{q}\right)$. To declare condition $\mathcal{E} j$ valid, first we require that conditions $\mathcal{E}_{s}$ hold, for $s<j$. In particular, condition $\mathcal{E}_{q}$ is valid. Take an open cover $\left\{V_{i q}\right\}$ of $C^{(0)}{ }_{q}$ (teh $q$-th algorithmic center in (1) of 9.1) such that on each $V_{i q}$ we have a nice $A$-basic object $B_{i q}^{\prime \prime}:=\left(B_{q \mid U_{i}}\right)^{\prime \prime}$ with adapted hypersurface $Z_{i q} \subset V_{i q}$ (i.e., $V_{i q}$ is amenable, see 8.3). From $\operatorname{dim}(M)<d-1$ we see that $Z_{i q}$ is inductive (i.e., (A3) of 6.2 holds, see 6.12) Next we take, for each $i$, the homogeneous $A$-basic object $\left(H B_{i q}^{\prime \prime}\right):=\mathcal{H}\left(B_{i q}^{\prime \prime}\right)$ (again nice), admitting $Z_{i q}$ as an inductive hypersurface. Consider next, for each index $i$, the inductive object $B_{i q}^{*}:=\left(H B_{i q}^{\prime \prime}\right)_{Z_{i q}}$ and the algorithmic equiresolution sequence

$$
\text { (3) } B_{i q}^{*} \leftarrow B_{i q+1}^{*} \leftarrow \cdots \leftarrow B_{i j}^{*} \leftarrow \cdots
$$

(obtained by the validity of condition $\mathcal{E}_{q}$ ) with centers $C_{i p} \subset u s\left(B_{p}^{*}\right), q \leq$ $p \leq j$. (It reaches at least level $j$ because of the equalities $\max \left(t_{q}\right)=\cdots=$ 
$\left.\max \left(t_{j}\right)\right)$. Finally, we say that $B$ satisfies condition $\mathcal{E}_{j}$ if the sequence (3) is strongly permissible (6.17), for all $i$. Then there is an induced permissible sequence $\left(H B_{i q}^{\prime \prime}\right) \leftarrow \cdots \leftarrow\left(H B_{i j}^{\prime \prime}\right)$. Let $\widetilde{B_{i q}}$ denote the restriction of $B_{q}$ to $V_{i q}$. By the W-equivalence of $\widetilde{B_{i q}}$ and $\left(H B_{i q}^{\prime \prime}\right)$ and repeated application of 8.4, we obtain (by using the same centers) an induced $t$-permissible sequence

$$
\text { (4) } \widetilde{B_{i q}} \leftarrow \widetilde{B_{i, q+1}} \leftarrow \cdots \leftarrow \widetilde{B_{i j}}
$$

From the validity of $\mathcal{E}_{p}$ for $p<j$, by induction we may assume that by varying $i$ the centers $C_{i p}$ glue together, to produce globally defined centers $C_{p}$, $q \leq p<j$, so that the resulting sequence coincides with (1). More precisely, we obtain $C_{q} \subset u s\left(B_{q}\right)$, next $C_{q+1} \subset u s\left(B_{q+1}\right)$, where $B_{q+1}=\mathcal{T}\left(B_{q}, C_{q}\right)$, and so on. Finally, we have $B_{j}=\mathcal{T}\left(B_{j-1}, C_{j-1}\right)$. In this way, $u s\left(\widetilde{B_{j}}\right)$ any be identified to an open $V_{i j} \subset u s\left(B_{j}\right)$, the center $C_{i j}$ of $B_{i j}^{*}$ to a closed subscheme of $V_{i j} \subset u s\left(B_{j}\right)$. Arguing as in 9.7(II), using Lemma 9.6 (now in case $\lambda=j$ ) we see that these agree on intersections. By the strong permissibility condition on the sequence (3), we obtain a $t$-permissible center $C_{j}$ of $B_{j}$. This $C_{j}$ is the center $\mathcal{E}_{j}$ attaches to $B_{j}$. Again by Lemma 9.6, this center is independent of the choice of the cover and the adapted surfaces selected, so $C$ is well defined.

Situation $(\beta)$ is treated exactly as case " $\operatorname{dim} M=d-1$ " in 9.7.

In both cases $\alpha$ and $\beta$ property (a) of 9.1 follows from the fact that our construction over $A$ induces on the fiber the appropriate algorithmic center in the resolution (1) of 9.1, (b) of 9.1 is immediate and (c), (d) are easily verified by using the remarks of 9.5 .

So, we have completed the definition of conditions $\mathcal{E}_{j}$, and the proof of Theorem 1.1 (see 9.2) is complete.

Example 9.9. A basic object over $A$ may have many equiresolutions, but only one of these can be the algorithmic equiresolution. Consider the following example. Here, $k$ denotes a characteristic zero field, $A=k[\epsilon]$, where $\epsilon^{2}=0, R=A[x, y]$ ( $x, y$ indeterminates), $S=\operatorname{Spec}(A), W=$ $\operatorname{Spec}(R), W \rightarrow S$ is induced by the natural homomorphism $A \rightarrow R$. Let $B=\left(W \rightarrow S,\left(y^{2}, x^{3}\right), 2, \emptyset\right)$. Then $B^{(0)}=\left(\operatorname{Spec}\left(k[x, y],\left(y^{2}, x^{3}\right), 2, \emptyset\right)\right.$ and $\operatorname{Sing}\left(B^{(0)}\right)=V(x, y)$ (the origin). If we blow-up the origin 0 of $\operatorname{Spec}(k[x, y])$ the resulting transform $B_{1}^{(0)}$ will satisfy $\operatorname{Sing}\left(B_{1}^{(0)}\right)=\emptyset$, and clearly this is the algorithmic resolution of $B^{(0)}$. Concerning $B$, if (for $\lambda \in k$ ) $C_{\lambda} \subset W$ is the subscheme defined by the ideal $I\left(C_{\lambda}\right):=(y, x-\lambda \epsilon) \subset R$, then $C_{\lambda}$ is a permissible center. Indeed, if we let $x^{\prime}:=x-\lambda \epsilon$, then $I=\left(y^{2},{x^{\prime}}^{3}+3 \lambda \epsilon x^{\prime 2}\right)$ and $I\left(C_{\lambda}\right)=\left(y, x^{\prime}\right)$. Now we immediately see that $\nu(I, C)=\nu\left(I^{(0)}, C^{(0)}\right)=2$. Clearly $C_{\lambda}$ induces the origin 0 on $B^{(0)}$, for all $\lambda$. If $B_{1 \lambda}$ is the transform $B$ 
with center $C_{\lambda}$, then $\operatorname{Sing}\left(B_{1 \lambda}\right)$ is nonsingular, for all $\lambda$. In fact, its restriction to the non trivial affine open of the blowing-up is ( $\operatorname{Spec}\left(A\left[x^{\prime}, y\right] \rightarrow\right.$ $\left.S,\left(y^{2}, x^{\prime}+3 \lambda \epsilon\right), 2, H\right)$ (with $\left.H=\mathcal{V}\left(x^{\prime}\right)\right)$, and we see that $\operatorname{Sing}\left(B_{1 \lambda}\right)=\emptyset$. Thus, for any $\lambda$ we obtain an equiresolution of $B$.

For what values of $\lambda$ will this be algorithmic, that is will condition $\mathcal{E}_{0}$ hold? We are in the case where (in the notation of 9.7) we have $\operatorname{codim}(M)>1$, so we have to use the inductive object. Note that $B$ is a nice $A$-basic object, $Z$ defined by the ideal $(y) A[x, y]$ is an inductive hypersurface. Let us study condition $\mathcal{E}_{0}$ using $Z$. Here, $\Delta(I / S)=\left(y, x^{2}\right), \mathcal{H}(I / S)=\left(y^{2}, x^{2} y, x^{3}\right)$, $\mathrm{C}(\mathcal{H}(I / S), Z)=\left(x^{3}\right) A[x]$. Hence, $B_{Z}=\left(\operatorname{Spec}\left(A[x] \rightarrow S,\left(x^{3}\right), 2, \emptyset\right)\right.$. By induction, to have condition $\mathcal{E}_{0}$ satisfied, we need a permissible center for $B_{Z}$. To study permissiblity for $C_{\lambda}$ (defined by $I\left(C_{\lambda}\right)=(x-\lambda \epsilon)$ ), note that $(x-\lambda \epsilon)^{3}=\left(x^{3}-3 \lambda \epsilon x^{2}\right)$. From this fact we see that for $\lambda \neq 0$ we get $2=\nu\left(J, C_{\lambda}\right)<\nu\left(J^{(0)}, C_{\lambda}^{(0)}\right)=3$, so that the center $C_{\lambda}$ is not permissible, while for $\lambda=0$ we get $\nu\left(J, C_{0}\right)=\nu\left(J^{(0)}, C_{0}^{(0)}\right)=3$, so that $C_{0}$ is permissible. So, this way the only algorithmic center we get is $C_{0}$, in agreement with the general theory

9.10. Proof of Lemma 9.6. We proceed by induction on $t$. Let $t=0$, i.e., we have a single $A$-basic object $(H B)$. Let $C$ (resp. $C^{\prime}$ ) be the algorithmic center of $(H B)_{Z}$ (resp. $\left.(H B)_{Z^{\prime}}\right)$. Firstly, we claim that $C^{(0)}=C^{(0)}$ (equality of fibers). Indeed, by (a) in 9.1, these are the algorithmic zeroth centers of the fibers $\left(H B^{(0)}\right)_{Z^{(0)}}$ and $\left(H B^{(0)}\right)_{Z^{\prime(0)}}$ respectively. But both centers, regarded as subschemes of $W^{(0)}:=u s\left(H B^{(0)}\right)$ must be the (unique) algorithmic zeroth center of $\left(H B^{(0)}\right)$ (by the construction when the base is a field). Hence they agree: $C^{(0)}=C^{\prime(0)}$.

Since the set of closed points is dense, to show the equality $C=C^{\prime}$ it suffices to show that if $y$ is any closed point in $C^{(0)}=C^{(0)}$, then the restrictions of $C$ and $C^{\prime}$ to a neighborhood of $y$ coincide.

By using 7.13 we find etale morphisms $\pi, \pi^{\prime}$ from a scheme $Y$ into $Z$ and $Z^{\prime}$ respectively, such that $\pi^{*}\left((H B)_{Z}\right)=\pi^{\prime *}\left((H B)_{Z^{\prime}}\right)$. By $(\mathrm{c})$ of 9.1 , both $\pi^{-1}(C)$ and $\pi^{\prime-1}\left(C^{\prime}\right)$ must be the algorithmic zeroth center that condition $\mathcal{E}_{0}$ assigns to $\left.\pi^{*}\left((H B)_{Z}\right)\right)$ and $\pi^{\prime *}\left((H B)_{Z^{\prime}}\right)$ respectively. Since these $A$-basic objects are equal, $\pi^{-1}(C)=\pi^{\prime-1}\left(C^{\prime}\right)$, whence $C=C^{\prime}$ near $y$, as claimed.

The inductive step (transition from $t$ to $t+1$ ) is accomplished with an argument similar to that used for $t=0$, applying the inductive hypothesis and again Theorem 7.13.

9.11. On generalized basic objects. Initially, we intended to use strictly the resolution process for basic objects (over a field) discussed in [11] or [5]. In it there is a fundamental inductive step, where one replaces a basic object $B$ first by a nice object $B^{\prime \prime}$ (locally defined) and then this one by an object $B_{Z}$ 
with underlying scheme $Z$, a regular hypersurface defined on $u s\left(B^{\prime \prime}\right)$. By induction we have a resolution function for $B_{Z}$, which allows us to define (locally) a resolution function for $B$. Alternately, we have an algorithmic permissible center for $B_{Z}$ which produces (locally) an algorithmic center for $B$. But this construction is local and moreover, given $B^{\prime \prime}$, there are many adapted hypersurfaces $Z$, so there is a problem of patching, if we try to get a globally defined resolution function (or algorithmic permissible resolution center). (Similar considerations apply to a basic object $B_{j}$ which appears in a suitable permissible sequence of basic objects, see 6.13). In these references to solve this problem the authors use generalized basic objects. In a suitable sense these are, locally, basic objects, but it is possible to define for them global permissible centers, globally defined $t$-functions (see 2.8), and so on. Once this is verified, the mentioned patching problem is easily solved. Key results to implement this approach are: (a) Hironaka's trick (see [5, Section 21]) (b) the fact that formula (1) of 6.13 holds or (essentially equivalently), (c) that a center $C$ is permissible for $B$ if and only if it is permissible for $B_{Z}$. As we saw, the statement analogous to (c) working over $A \in \mathcal{A}$ rather than a field is not true (see 6.14 and 6.15). Hence it does not seem possible to adapt this approach to the situation where we work over an artinian ring, necessary for Theorem 1.1. This difficulty was overcome by abandoning generalized basic objects and using some techniques from [28] instead. This leads to the VW-resolution algorithm, that we have used. However, many aspects of the theory of generalized basic objects, including Hironaka's trick, can be adapted to the case of objects over $A \in \mathcal{A}$. Since we do not make use of these results, we do not present the details here.

\section{Families of ideals and varieties}

It is known that, working over a field, our algorithm of resolution for basic objects induces algorithms for principalization of ideals and resolution of embedded varieties (see [5]). In this section we present a brief review of these facts and a description of an analogue when we work over an Artin ring. Throughout, $A$ will denote a ring in the class $\mathcal{A}$ of $3.1, S:=\operatorname{Spec}(A)$.

10.1. Families of ideals. Given an Artin ring $A \in \mathcal{A}$, an idealistic triple (or id-triple) over $A$ is a 3 -tuple $\mathcal{T}=(p: W \rightarrow S, I, E), S=\operatorname{Spec}(A)$, such that $(W \rightarrow S, E)$ is an $S$-pair (3.5) and $I$ is a never-zero $W$-ideal (2.1).

There is natural notion of (closed) fiber, by reducing modulo $r(A)$. This fiber is an id-triple over $k=A / r(A), \mathcal{T}^{(0)}=\left(W^{(0)} \rightarrow \operatorname{Spec} k, I^{(0)}, E^{(0)}\right)$. We might call an id-triple over $A \in \mathcal{A}$ a family of triples (over fields) parametrized by $S$ or an infinitesimal deformation of the triple $\mathcal{T}^{(0)}$. If $A=k$ is a field, often we shall write $(W, I, E):=(W \rightarrow \operatorname{Spec} k, I, E)$. 
Given and id-triple $\mathcal{T}=(W \rightarrow S, I, E)$ and a subscheme $C$ of $W$ which is a permissible center for the underlying $S$-pair $(W \rightarrow S, E)(3.5)$, we may define the transform of $\mathcal{T}$ with center $C$. This is the id-triple $\mathcal{T}_{1}=\left(W_{1} \rightarrow\right.$ $\left.S, I_{1}^{\prime}, E_{1}\right)$, where $\left(W_{1} \rightarrow S, E_{1}\right)$ is the transform of the $S$-pair $(W \rightarrow S, E)$ with center $C$ (3.12) and $I_{1}^{\prime}=I \mathcal{O}_{W_{1}}$ (the total transform of $I$ to $W_{1}, 4.5$ ).

An $A$-resolution, or equiprincipalization of $\mathcal{T}$ is a sequence $\mathcal{T}=\mathcal{T}_{0} \leftarrow$ $\mathcal{T}_{1} \leftarrow \cdots \leftarrow \mathcal{T}_{r}$ of id-triples, with $\mathcal{T}_{i}=\left(W_{i} \rightarrow S, I_{i}, E_{i}\right), \mathcal{T}_{i+1}$ the transform of $\mathcal{T}_{i}$ with a permissible center, such that $I_{r}^{\prime}=I\left(H_{1}\right)^{c_{1}} \ldots I\left(H_{s}\right)^{c_{s}}$ (where $\left.E_{r}=\left(H_{1}, \ldots, E_{s}\right)\right)$, for suitable (locally constant) integral exponents $c_{i} \geq 0$, $i=i, \ldots, s$. By taking fibers, such an $A$-principalization induces a principalization sequence for $\mathcal{T}^{(0)}$ (see [5], Theorem 2.5).

It is known that when the base is a field $k$ (of characteristic zero) our algorithm of resolution for basic objects induces an algorithm for principalization of id-triples. Namely, given the id-triple $\mathcal{T}=(W, I, E)$ over a field $k$, one considers the basic object $B_{0}=(W, I, 1, E)$ and applies the algorithm to $B_{0}$, getting a resolution $B=B_{0} \leftarrow \cdots \leftarrow B_{r}, B_{i}=\left(W_{i}, I_{i}, 1, E_{i}\right)$. Just by dropping the entry $b=1$ in each basic object $B_{i}$, we get the desired principalization. See [5, Parts I, II] for details. Henceforth this principalization process will be referred to as the algorithmic principalization of $\mathcal{T}=(W, I, E)$.

Now, relative to the VW-algorithm, we introduce a notion of algorithmic equiresolution, or equiprincipalization, for id-triples over $A \in \mathcal{A}$. Namely, given such an id-triple $T=(W \rightarrow S, I, E)$, with fiber $\mathcal{T}^{(0)}=\left(W^{(0)}, I^{(0)}, E^{(0)}\right)$, we say that $\mathcal{T}$ is algorithmically equiprincipalizable if conditions $\mathcal{E}_{0}, \ldots, \mathcal{E}_{r-1}$ are valid for the basic object $B=(W \rightarrow S, I, 1, E)$. Alternatively, we could demand that conditions $\mathcal{E}_{j}$ be valid for all possible $j$, since they are vacuously hold for $j \geq r$. So, if $\mathcal{T}$ is equiprincipalizable, we have an algorithmic equiresolution $B=B_{0} \leftarrow \cdots \leftarrow B_{r}, B_{i}=\left(W_{i}, I_{i}, 1, E_{i}\right)$, of $B$. As before (when we worked over a field), by dropping throughout the entry $b=1$, we obtain an $A$-principalization of $\mathcal{T}$ inducing by taking fibers the algorithmic principalization of $\mathcal{T}^{(0)}$. This will be called the algorithmic equiprincipalization of $\mathcal{T}$.

It is easy to state and prove a theorem analogous to 1.1 for $A$-triples, $A \in \mathcal{A}$. We leave this task to the reader.

We could have defined the notion of family of ideals parametrized by $\operatorname{Spec}(A), A \in \mathcal{A}$ as a pair $(p: W \rightarrow S, I)$, with $p$ and $I$ as above, and essentially repeat what was done above. But since in an $A$-resolution process the exceptional divisors that appear must be considered, it seems more reasonable to work from the outset with id-triples instead.

10.2. Working over a characteristic zero field $k$, a pair $\mathcal{X}=(X, W)$ where $W$ is a scheme, smooth over $k$, and $X$ is a reduced, equidimensional subscheme of $W$ will be called an embedded variety. 
A resolution of $\mathcal{X}$ is a proper, birational morphism $f: W^{\prime} \rightarrow W$, with $W^{\prime}$ smooth, such that: $(i)$ the exceptional locus of $f$ is the union of regular hypersurfaces $H_{1}, \ldots, H_{n}$ with normal crossings, $(i i)$ the strict transform $X^{\prime}$ of $X$ to $W^{\prime}$ is regular, and has normal crossings with $H_{1}, \ldots, H_{n}$, (iii) $f$ induces an isomorphism $X^{\prime}-f^{-1}(\Sigma) \rightarrow X-\Sigma$, where $\Sigma$ is the singular locus of $X$.

As explained in Sections (2.4) and (5.8) of [5], our algorithm for resolution of basic objects induces an algorithm for resolution of embedded varieties. Indeed, consider the basic object $B=(W, I(X), 1, \emptyset)$ and its corresponding algorithmic resolution:

$$
B=B_{0} \leftarrow B_{1} \leftarrow \cdots \leftarrow B_{r}
$$

obtained via resolution functions $g_{0}, \ldots, g_{r-1}$, taking values in a totally ordered set $\Lambda^{(d)}, d=\operatorname{dim}(W)$ (see 2.8). We write $B_{i}=\left(W_{i}, I_{i}, 1, E_{i}\right)$, for all $i$. A property of our algorithm says that $g_{0}$ is constant, say $=a \in \Lambda^{(d)}$ on $W \backslash \operatorname{Sing}(X)$ and there is a unique index $n$ (depending on $B$, hence on $\mathcal{X}$ ) such that $\max \left(g_{n}\right)=a$. It turns out that the strict transform $X_{n}$ of $X$ to $W_{n}$ is a union of components of $\operatorname{Max}\left(g_{n}\right)$ (i.e., the $n$-th center in the algorithmic resolution process), hence it is regular, having normal crossings with $E_{n}$. We shall denote the index $n$ above by $\eta(\mathcal{X})$.

10.3. Relative $A$-varieties and their resolutions. If $A \in \mathcal{A}(3.1)$, a scheme $X$ together with a flat morphism of finite type $f: X \rightarrow S=\operatorname{Spec} A$ will be called a relative $A$-scheme. The morphism won't be specified when it is clear from the context. If the (only) fiber $X^{(0)}$ is reduced and equidimensional, we shall talk about a relative $A$-variety. In that case, if $U$ is the open set of points where $X^{(0)}$ is smooth over $k=A / r(A)$, the induced morphism $X_{\mid U} \rightarrow S$ is smooth over is smooth (because it is flat, with smooth fiber). Note that $X_{\mid U}$ is a Cohen-Macaulay scheme (use 21.C, page 154, in [19]). Let us write $\mathcal{S}(X / S):=X_{\mid U}$.

A resolution of an a relative $A$-variety $f: X \rightarrow S$ is a proper morphism $\phi: X^{\prime} \rightarrow X$ such that: $(i) f \phi: X^{\prime} \rightarrow S$ is smooth, $(i i) \phi$ induces a resolution of fibers morphism $\phi^{(0)}: X^{\prime(0)} \rightarrow X^{(0)}$ (i.e., $\phi^{(0)}$ is proper, birational and an isomorphism off the singular locus of $\left.X^{(0)}\right)$.

10.4. Relative embedded $A$-schemes and varieties. As usually, $A$ is an Artin $\operatorname{ring}$ in $\mathcal{A}, S=\operatorname{Spec}(A)$.

(a) A relative embedded $A$-scheme (or an embedded scheme, flat over $S$ ) is a pair $\mathcal{X}=(X, p: W \rightarrow S)$, with $p$ smooth, $X$ a closed subscheme of $W$, such that the morphism $q: X \rightarrow S$ induced by $p$ is flat.

If in addition $X^{(0)}$ is reduced and equidimensional, we talk about a relative embedded $A$-variety. In this case $\left(X^{(0)}, W^{(0)}\right)$ is an embedded variety over $k=A / r(A)$. 
(b) We say that an the relative embedded $A$-variety $\mathcal{X}$ is equisolvable if there is a proper morphism $\psi: W^{\prime} \rightarrow W$ such that: $(i)$ the composition $p \psi: W^{\prime} \rightarrow S$ is smooth, (ii) $\psi$ induces a proper birational morphism of fibers $W^{\prime(0)} \rightarrow W^{(0)}$, (iii) If $\tilde{X}=\mathcal{S}(X / A)$ (the largest open subscheme of $X$ smooth over $S$, see 10.3) and $X^{\prime}$ is the scheme-theoretic closure of $\psi^{-1}(\tilde{X})$ in $W^{\prime}$, then the induced morphism $X^{\prime} \rightarrow X$ is a resolution of the relative $A$-variety $q: X \rightarrow S$.

We have used the term relative by analogy with the well established terminology concerning divisors flat over a base scheme, see [22, page 72].

Theorem 10.5. Let $\mathcal{X}=(X, p: W \rightarrow S)$ be a relative $A$-embedded variety, $\mathcal{X}^{(0)}=\left(X^{(0)}, W^{(0)}\right)$ its fiber. Assume $B:=(p: W \rightarrow S, I(X), 1, \emptyset)$, satisfies conditions $\mathcal{E}_{0}, \ldots, \mathcal{E}_{q}$, where $q=\eta\left(\mathcal{X}^{(0)}\right)$ (10.8). Then, $\mathcal{X}$ is equisolvable in the sense of $10.4(b)$.

Proof. The validity of these conditions implies the existence of a permissible sequence

$$
B=B_{0} \leftarrow \cdots \leftarrow B_{q} \leftarrow B_{q+1}
$$

$B_{i}=\left(W_{i}, I(X)_{i}, 1, E_{j}\right)$, with centers $C_{i} \subset W_{i}, i=0, \ldots q$, inducing on special fibers the $(q+1)$-truncation of the algorithmic resolution sequence of the fiber $B^{(0)}=B_{0}^{(0)}$ (with centers $C_{i}^{(0)}, i=0, \ldots, q$ ). By 10.2 the strict transform $X_{q}^{(0)}$ of $X$ to $u s\left(B_{s}^{(0)}\right)$ is the (disjoint) union of components $Y_{1}^{(0)}, \ldots, Y_{s}^{(0)}$ of the center $C_{q}^{(0)}$. Let $Y_{i}, i=0, \ldots, s$ be the component of the center $C_{q}$ inducing $Y_{i}^{(0)}$ on the special fiber (i.e., modulo $r(A)$ ) and $\psi: W_{q} \rightarrow W_{0}$ the naturally induced morphism. Using the fact that $\mathcal{S}(X / A)$ is a Cohen-Macaulay scheme and remark (b) in the proof of Proposition 3.10, one sees that the scheme -theoretic closure of $\psi^{-1}(\mathcal{S}(X / A))$ in $W_{q}$ is equal to $Y_{1} \cup \ldots \cup Y_{s}:=X_{q}$. Since $C_{q}$ is smooth over $S$ (via the restriction of the natural projection $\left.W_{q} \rightarrow S\right) X_{q}$ is smooth over $S$ and one readily checks that the morphism $\psi: W_{q} \rightarrow W$ defines an equiresolution of $\mathcal{X}$ in the sense of 10.4 (b).

10.6. In the notation of Theorem 10.5 , when conditions $\mathcal{E}_{0}, \ldots, \mathcal{E}_{q}, q=$ $\eta\left(\mathcal{X}^{(0)}\right)$, hold for $B=(p: W \rightarrow S, I(X), 1, \emptyset)$ we say that the relative embedded $A$-variety $\mathcal{X}$ is algorithmically equisolvable, and call the equiresolution obtained in the Proof of 10.5 its algorithmic equiresolution.

10.7. We use the notation of 10.5 and its proof. If $\mathcal{X}$ is is algorithmically equisolvable the algorithmic equiresolution of 10.5 has some additional properties, namely: (a) the morphism $\Psi: W_{q} \rightarrow W$ is a composition of blowing-ups with centers smooth over $S$, (b) the exceptional divisor $D$ of $\psi$ is a union of hypersurfaces with normal crossings (3.4), (c) $X_{q}$ has normal crossings with $D$. 
10.8. To finish this chapter we indicate how our notions of equiresolution (for basic objects, ideals or embedded schemes), given when we work over $S=\operatorname{Spec}(A), A \in \mathcal{A}$, naturally induce similar notions when working with families parametrized by an arbitrary noetherian scheme $T$. Consider, e.g., a basic object over a scheme $T$, that is a four-tuple $B=(p: W \rightarrow T, I, b, E)$, with $p$ a smooth morphism, $I$ a never-zero ideal of $\mathcal{O}_{W}, b$ a positive integer, $E$ a finite sequence of distinct hypersurfaces of $W$ with normal crossings. Let $t \in T, R_{t}:=\mathcal{O}_{T, t}$ and $P_{t}$ the maximal ideal of $R$. Hence, for any non-negative integer $m, R_{t, m}:=R / P_{t}^{m+1} \in \mathcal{A}$. The family $B$ induces an $R_{t, m}$-basic object $B_{t, m}=\left(p_{t, m}: W_{t, m} \rightarrow S_{t, m}, I_{t, m}, b, E_{t, m}\right)\left(S_{t, m}=\operatorname{Spec}\left(R_{t, m}\right)\right)$ where the morphism $p_{t, m}$ is obtained by base change (via the natural morphism $S_{t, m} \rightarrow T$ ), the hypersurfaces in $E_{t, m}$ come, by pull-back, from those in $E$, and $I_{t, m}=I \mathcal{O}_{W_{t, m}}$. We say that $B$ is algorithmically equisolvable at $t$ if, for every integer $m \geq 0$, the induced family $B_{t, m}$ is an equisolvable $R_{t, m^{-}}$ basic object, in the sense of 9.3. Finally we say that $B$ is algorithmically equisolvable if it is algorithmically equisolvable at $t \in T$, for every $t \in T$.

Similarly, essentially by substituting in our previous work (in 10.1 and 10.4) the base $S=\operatorname{Spec}(A)$ by an arbitrary noetherian scheme $T$, we introduce the notions of family of id-triples and family of embedded schemes, parametrized by $T$. With the notation above, we naturally obtain induced families over $R_{t, m}$, at each point $t \in T$ and we say that a family of id-triples is equiprincipalizable at $t$ is the induced family over $R_{t, m}$ is so, for all $m \geq 0$. Finally we say that it is equiprincipalizable if it is equiprincipalizable at every $t \in T$.

Following [11] or [5] we say that a family of embedded schemes $(X, W \rightarrow S)$ is algorithmically equisolvable if its associated family of id-triples ( $W \rightarrow$ $S, I(X), b, \emptyset)$ is algorithmically equiprincipalizable.

In case the parameter space is a smooth algebraic $k$-scheme $T$ ( $k$ a characteristic zero field) these notions are closely related to those studied in [10] or [5, Section 10]. This will be discussed in a subsequent article. We hope that that the present theory will also have applications similar to those considered in [10, Section 4] (Hilbert schemes).

\section{Appendix 1: Review of useful results}

In this appendix we collect a number of basic algebraic and geometric results that are used in the paper. Probably most of them are well known, but we prove those for which we could not find appropriate references in the literature.

11.1. (a) We shall use the notation and terminology of 3.1. Thus, $\mathcal{A}$ denotes the collection of artinian local rings $(A, \mathcal{M})$ such that the residue field $k=$ 
$A / \mathcal{M}$ has characteristic zero. Any ring in $\mathcal{A}$ is necessarily a complete $k$ algebra, $k=A / r(A)$.

(b) We shall be primarily concerned with the following situation: $(A, \mathcal{M})$ is an artinian ring in $\mathcal{A},(R, \mathcal{N})$ a local noetherian $A$-algebra, essentially of finite type (via a local homomorphism $A \rightarrow R$ ), $S=\operatorname{Spec}(A)$. Let $Z=\operatorname{Spec}(R)$ and $\pi: Z \rightarrow S$ be the induced morphism. The closed fiber is isomorphic to $\operatorname{Spec}\left(R^{(0)}\right)$, where $R^{(0)}:=R / \mathcal{M} R$

(c) Often it will be the case that $R$ is a $\mathcal{N}$-smooth $A$-algebra, or $\mathcal{N}$ smooth over $A$. This is usually defined in terms of a homomorphism lifting property (see [20, page 213] or [1, Def. 14, p. 222]). Equivalently, this means that that the morphism $\pi$ above is flat, with its closed fiber geometrically regular, see $[1$, Thm. 18, p. 224]. Under our assumption that the field $A / \mathcal{M}$ has zero characteric, this just means: "the closed fiber is regular", that is " $R^{(0)}$ is regular".

Lemma 11.2. With notation as in $11.1(b)$, assume moreover that $R^{(0)}$ is a regular ring, of dimension $n, a_{1}^{(0)}, \ldots, a_{n}^{(0)}$ is a regular system of parameters of $R^{(0)}$, and for all $i$ let $a_{i} \in \mathcal{N}=r(R)$ be such that $a_{i}$ induces $a_{i}^{(0)}$ via the canonical homomorphism $R \rightarrow R^{(0)}$. Then: $(i) a_{1}, \ldots, a_{n}$ is a regular sequence in $R$, (ii) $R$ is a Cohen-Macaulay ring, (iii) If $R$ is a $\mathcal{N}$-smooth $A$-algebra then, for any indices $1 \leq i_{1}<\cdots<i_{r} \leq n, R /\left(a_{i_{1}}, \ldots, a_{i_{r}}\right) R$ is a $\mathcal{N}$-smooth A-algebra.

Proof. We show that $a_{1}, \ldots, a_{n}$ is a regular sequence by induction on $n$, the case $n=0$ (i.e., $A=R$ ) being trivial. It suffices to check that $a:=a_{1}$ is a regular element of $R$. In fact, if this is the case, then our hypotheses applies to $R /\left(a_{1}\right)$ and the images of $a_{2}, \ldots, a_{n}$ in this ring, and we use induction.

We shall see that $a$ is regular in $R$ by induction on $\operatorname{dim}(A)$ (dimension as a vector space over $k=A / \mathcal{M})$. The case where $\operatorname{dim}(A)=0$ is trivial. For the inductive step, we shall use the well-known fact that the maximal ideal $\mathcal{M}$ contains an element $\epsilon \neq 0$ such that $\epsilon \mathcal{M}=0$. Let $A^{\prime}:=A /(\epsilon) A$, $\phi=R \rightarrow R^{\prime}:=R /(\epsilon) R$ be the canonical homomorphism and $b$ the image of $a_{1}$ in $R^{\prime}$. By induction assumption $b$ is a regular element. Let $\alpha$ be an element of $R$ such that $a \alpha=0, \alpha^{\prime}$ its image in $R^{\prime}$. Then, $b \alpha^{\prime}=0$, which (by the regularity of $b$ ) implies that $\alpha^{\prime}=0$. Hence, $\alpha \in K:=\operatorname{Ker}(\phi)=(\epsilon) R$. Note that, by the property of the element $\epsilon, K=(\epsilon) R$ is naturally a $k$-module, as such isomorphic to $R^{(0)}$. By this isomorphism, $\alpha \in K$ corresponds to $c \in R^{(0)}$, and $a \alpha$ to $a_{1}^{(0)} c$. Since $a_{1}^{(0)} \neq 0$ in the integral domain $R^{(0)}$ (because $a_{1}^{(0)}$ is part of a regular system of parameters), $c=0$, hence $\alpha=0$, as desired. This proves $(i)$ 
(ii) The fact that $R$ is Cohen Macaulay follows from the equalities $\operatorname{dim}(R)=\operatorname{dim}\left(R^{(0)}\right)=n$ and the existence of the regular sequence $a_{1}, \ldots, a_{n}$, that we just verified.

(iii) Concerning the smoothness, to begin with $R$ is $A$-flat by the "lifting relations criterion" ([3] p. 11, the proof presented there, for polynomial rings, works more generally and yields the result we need here.) In fact, we are dealing with regular sequences, whose relations are trivial. Next, the only closed fiber is a regular variety, since $a_{1}^{(0)}, \ldots, a_{n}^{(0)}$ was a regular system of parameters in the regular local ring $R^{(0)}$, which gives us smoothness.

Proposition 11.3. Let $f: X \rightarrow Y$ be a morphism of noetherian schemes, $D \subset X$ an effective Cartier divisor (which we identify to a closed subscheme of $X$ locally defined by a non-zero divisor), $U=X-D, f^{\prime}: D \rightarrow Y$ and $f_{U}: U \rightarrow Y$ the morphisms induced by $f$ by restriction. Assume both $f^{\prime}$ and $f_{U}$ are smooth. Then, $f$ is smooth (i.e., flat with geometrically regular fibers).

Proof. In [23] it is shown that the flatness of $f^{\prime}$ and $f_{U}$ implies that $f$ is flat. Let us check that all the geometric fibers are regular. So, consider such a fiber $X_{y}, y$ a geometric point of $Y$. Here, with obvious notation, $D \cap X_{y} \subset X_{y}$ is a Cartier divisor, let $U_{y}=X_{y}-D$. Note that $D \cap X_{y}$ (resp. $U_{y}$ ) can be identified to the fiber of $f^{\prime}$ (resp. $f_{U}$ ) at $y$, hence is regular. Then the regularity of $X_{y}$ is a consequence of the following lemma, finishing the proof.

Lemma 11.4. Let $(R, \mathcal{N})$ be a noetherian local ring, $a \in \mathcal{N}$ a regular element of $R$ (i.e., a non-zero divisor), suppose $R /(a)$ is a regular ring. Then, $R$ is regular.

Proof. Let $\operatorname{dim}(R)=d, R^{\prime}:=R / \mathcal{N}, \mathcal{N}^{\prime}$ the maximal ideal of $R^{\prime}$. By [4, Cor. 12.18], $\operatorname{dim}\left(R^{\prime}\right)=d-1$, The natural homomorphism of vectors spaces (over the common residue field $k) \mathcal{N} / \mathcal{N}^{2} \rightarrow \mathcal{N}^{\prime} / \mathcal{N}^{\prime 2}$ is onto and its kernel is generated by $a+\mathcal{N}^{2}$, hence we have $\operatorname{dim}\left(\mathcal{N} / \mathcal{N}^{2}\right) \leq \operatorname{dim}\left(\mathcal{N}^{\prime} / \mathcal{N}^{\prime 2}\right)+1$, where dim means dimension as a vector space. But by the assumed regularity of $R^{\prime}$, the right hand side is equal to $\operatorname{dim}\left(R^{\prime}\right)+1=\operatorname{dim}(R)-1+1=\operatorname{dim}(R)=d$. Hence $\operatorname{dim}(R)=\operatorname{dim}\left(\mathcal{N} / \mathcal{N}^{2}\right)$ and $R$ is regular.

Proposition 11.5. The notation is as in 1.1(a), we asume that $R$ is $\mathcal{N}$ smooth over $A$. Let $a_{1}, \ldots, a_{n}$ be elements of $R$ inducing a regular system of parameters $a_{1}^{\prime}, \ldots, a_{n}^{\prime}$ of the regular local ring $R^{(0)}$ and $I=\left(a_{1}, \ldots, a_{r}\right) R$, $r \leq n$. If $p: Z^{\prime} \rightarrow Z$ is the blowing-up of $Z$ with center $I$ and $\pi^{\prime}: Z^{\prime} \rightarrow S$ the composition of $p$ and $\pi$, then $\pi^{\prime}$ is a smooth morphism. 
Proof. Let $R_{i}:=R\left[a_{1} / a_{i}, \cdots, a_{n} / a_{i}\right]$. By the usual local description of the blowing-up, it suffices to show that if we regard $R_{i}$ as an $A$-algebra via the composition homomorphism $A \rightarrow R \rightarrow R_{i}$, then the resulting morphism $U_{i}:=\operatorname{Spec}\left(R_{i}\right) \rightarrow S$ is smooth, for all $i=1, \ldots, n$. To simplify the notation take $i=1$. Let $E \subset Z^{\prime}$ be the exceptional divisor of our blowing-up, $E_{i}:=E \cap U_{i}$ (note that that $U_{i}$ is naturally identified to an open of $Z^{\prime}$ ). By Proposition 11.3, it suffices to show that the induced morphisms from $U_{i}^{\prime}:=U_{i}-E_{i}$ and $E_{i}$ to $S$ are both smooth. Since $U_{i}^{\prime}$ is isomorphic to $Z-V(I)$, and $Z$ is smooth over $S, U_{i}^{\prime} \rightarrow S$ is smooth. Concerning, $E_{i} \rightarrow S$, since $a_{1}, \ldots, a_{r}$ is a regular sequence in $R$, then we have an isomorphism of graded rings $\operatorname{gr}_{I}(R)=(R / I)\left[T_{1}, \ldots, T_{r}\right]$, where the $T_{i}$ 's are indeterminates. The Proj of this is $E$ and, "dehomogenizing", $E_{i}$ is isomorphic to $\operatorname{Spec}(R / I)\left[t_{2}, \ldots, t_{r}\right]$ ) (see [18], p. 152), where $t_{i}=T_{i} / T_{1}, i=2, \ldots, r$. Since $(R / I)\left[t_{2}, \ldots, t_{r}\right]$ is a polynomial ring over $R / I$ and $R / I$ is smooth over $A$ (by Lemma 11.2, iii), the smoothness of the projection $E_{i} \rightarrow S$ follows.

On the following proposition we use the notation of 3.1-3.3.

Proposition 11.6. Let $W \rightarrow S=\operatorname{Spec}(A)$ be a smooth morphism, $w \in$ $W, a_{1}, \ldots, a_{n}$ a partial system of A-regular parameters of $R=\mathcal{O}_{W, w}, I=$ $\left(a_{1}, \ldots, a_{n}\right) R$. Let $\hat{R}$ be the $I$-completion of $R$. Then, $\hat{R}$ is isomorphic to a power series ring $A^{\prime}\left[\left[x_{1}, \ldots, x_{n}\right]\right]$, so that the isomorphism sends $a_{i}$ into $x_{i}$, $i=1, \ldots, n$ and $A^{\prime}$ is isomorphic to $R / I$.

Proof. $(i)$ First we shall prove this result with the added assumption that $R$ contains a subring $A^{\prime}$ isomorphic to $R / I$ via the canonical quotient homomorphism. In this case $R$ is not necessarily local, and $a_{1}, \ldots, a_{n}$ may be assumed to be just a regular sequence.

Consider the ring homomorphism $\psi: A^{\prime}\left[x_{1}, \ldots, x_{n}\right] \rightarrow R$ (where the elements $x_{1}, \ldots, x_{n}$ are algebraically independent over $A^{\prime}$ ) such that $\psi(b)=b$ if $b \in A^{\prime}$ and $\psi\left(x_{i}\right)=a_{i}$ for all $i$. We claim that the induced homomorphism of completions, with respect to the ideals $\left(x_{1}, \ldots, x_{n}\right)$ and $\left(a_{1}, \ldots, a_{n}\right)$ respectively, is the desired isomorphism. It suffices to show that for all positive integer $j$ the homomorphism $\psi_{n}: A^{\prime}\left[x_{1}, \ldots, x_{n}\right] /\left(x_{1}, \ldots, x_{n}\right)^{j} \rightarrow R / I^{j} A^{\prime}$ induced by $\psi$ is bijective. By taking quotients this will be true if the induced homomorphism $\left(x_{1}, \ldots, x_{n}\right)^{j} /\left(x_{1}, \ldots, x_{n}\right)^{j+1} \rightarrow I^{j} / I^{j+1}$ is bijective, for all positive integer $j$. But the later statement is true by the isomorphism of graded rings $R / I\left[x_{1}, \ldots, x_{n}\right] \approx \mathrm{g} r_{I}(R)$ (proved, e.g., in [18, page 152]).

(ii) Let us consider now the general case. First let us check that the completion $\hat{R}$ contains a subring $A^{\prime}$ mapping isomorphically onto $\hat{R} / \hat{I} \approx R / I$ via the quotient map (where $\hat{I}:=I \hat{R}$ ). To see this, consider the commutative 
diagram

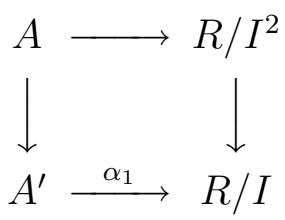

(recall that $R$ is an $A$-algebra). Note that by $11.2, A^{\prime} \approx R / I$ is smooth over $A$, hence the is a homomorphism $\alpha_{2}: A^{\prime} \rightarrow R / I^{2}$ making the resulting augmented diagram commutative. Thus, we obtain a commutative diagram:

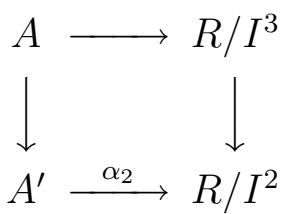

As before, the smoothness of $A^{\prime}$ over $A$ gives us a homomorphism $\alpha_{3}$ : $A^{\prime} \rightarrow R / I^{3}$ making the resulting augmented diagram commutative. Reiterating, we get a system of compatible homomorphisms $\alpha_{j}: A^{\prime} \rightarrow R / I^{j}$ for all positive integer $j$, which yields a homomorphism $A^{\prime} \rightarrow \hat{R}$ inducing an isomorphism $A^{\prime} \approx \hat{R} / \hat{I}$. Thus the image of $A^{\prime}$ (still denoted by $A^{\prime}$ ) is the desired subring of $\hat{R}$.

(iii) Now apply the result of $(i)$ to $\hat{R}$. If $R^{*}$ denotes its completion with respect to the ideal $\left(a_{1}, \ldots, a_{n}\right)$, then $R^{*}$ is isomorphic to a power series ring $A^{\prime}\left[\left[x_{1}, \ldots, x_{n}\right]\right]$, so that the isomorphism sends $a_{i}$ into $x_{i}$. But since $\hat{R}$ is $\left(a_{1}, \ldots, a_{n}\right)$-complete, the natural homomorphism $\hat{R} \rightarrow R^{*}$ is an isomorphism. Thus, $\hat{R}$ has the desired property, and the proposition is proved.

Next we present a result on blowing-ups that we use several times.

Proposition 11.7. Let $f: X \rightarrow T$ be a morphism of schemes, $C \subset X$ a close subscheme, flat over $T, J=I(C)$, such that $J_{x} \subset \mathcal{O}_{X, x}$ is generated by a regular sequence, for all $x \in C$. Let $T^{\prime} \rightarrow T$ be a morphism, $X^{\prime}=X \times_{T} T^{\prime}$, $p: X^{\prime} \rightarrow X$ the natural projection, $C^{\prime}=C \times_{T} T^{\prime}=p^{-1}(C), X_{1} \rightarrow X$ and $X_{1}^{\prime} \rightarrow X_{1}$ the blowing-ups with centers $C$ and $C^{\prime}$ respectively. Then, there is a natural isomorphism $X_{1}^{\prime}=X_{1} \times_{X} X^{\prime}$

11.8. In particular, we may take as $T^{\prime}$ a closed point $t$ of $T$ and the natural morphism Spec $(k(t)) \rightarrow T$. By the proposition, we may identify the blowing -up of $f^{-1}(t)$ with center $f^{-1}(t) \cap C$ with $f_{1}^{-1}(t)$, where $f_{1}: X_{1} \rightarrow T$ is obtained by composition.

By using the definition (or construction ) of the blowing-up given in [13, page 163], Proposition 11.7 is an easy consequence of the following algebraic lemma. In it, if $B$ is a ring and $I$ and ideal of $B$, we write $\mathcal{P}_{B}(I):=$ $B \oplus I \oplus I^{2} \oplus \cdots($ a graded $B$-algebra $)$. 
Lemma 11.9. Let $R \rightarrow B$ be a homomorphism of rings, $I \subset B$ an ideal, generated by a regular sequence, such that $B / I$ is $R$-flat. Let $R \rightarrow R^{\prime}$ a ring homomorphism, $B^{\prime}:=R^{\prime} \otimes_{R} B$ (naturally a $B$-algebra) and $I^{\prime}:=I B^{\prime}$. Then, $\mathcal{P}_{B} \otimes R^{\prime}=\mathcal{P}_{B^{\prime}}\left(I^{\prime}\right)$.

Proof. The contention follows if we prove that $I^{n} \otimes_{R} R^{\prime}=\left(I^{\prime}\right)^{n}=(I B)^{n}$, for all $n \geq 0$. But this follows if $B / I^{n}$ is $R$-flat, for all $n$. Indeed, from the exact sequence of $R$-modules

$$
0 \rightarrow I^{n} \rightarrow B \rightarrow B / I^{n} \rightarrow 0
$$

by tensoring with $R^{\prime}$ over $R$ we get, from the flatness of the $R$-module $B / I^{n}$, that the sequence

$$
0 \rightarrow I^{n} \otimes_{R} R^{\prime} \stackrel{\phi_{n}}{\longrightarrow} B^{\prime} \longrightarrow\left(B / I^{n}\right) \otimes_{r} R^{\prime} \rightarrow 0
$$

is exact. So, $\phi_{n}$ is injective and $I^{n} \otimes_{R} R^{\prime}=\operatorname{Im}\left(\phi_{n}\right)=\left(I B^{\prime}\right)^{n}$, as needed.

To see that $B / I^{n}$ is $R$-flat (for all $n \geq 0$ ), from the fact that $I$ is generated by a regular sequence, we obtain canonical isomorphisms

$$
\operatorname{gr}_{I}(B)=B / I \oplus I / I^{2} \oplus I^{2} / I^{3} \oplus \cdots=(B / I)\left[T_{1}, \ldots, T_{r}\right]
$$

(a polynomial ring, see [18, Proposition 5.10]). Thus, $I^{n} / I^{n+1}$ is a finite free $B / I$-module, for each $n$. Since $B / I$ is $R$-flat, it follows that $I^{n} / I^{n+1}$ is $R$-flat, for all $n \geq 0$. By using the exact sequences

$$
0 \rightarrow I^{n} / I^{n+1} \rightarrow B / I^{n+1} \rightarrow B / I^{n} \rightarrow 0
$$

and induction, we obtain that $B / I^{n}$ is $R$-flat for all $n \geq 0$, as desired.

\section{References}

[1] André, M.: Homologie des algèbres commutatives. Die Grundlehren der mathematischen Wissenschaften 206. Springer-Verlag, Berlin-New York, 1974.

[2] Artin, M.: Algebraic approximations of structures over complete local rings. Inst. Hautes Études Sci. Publ. Math. 36 (1969), 23-58.

[3] Artin, M.: Deformations of singularities. Tata Institute, Bombay, 1976.

[4] Atiyah, M. And Macdonald, I. G.: Introduction to commutative algebra. Addison-Wesley, Reading, Mass.-London-Don Mills, Ont., 1969.

[5] Bravo, A., Encinas, S. and Villamayor, O.: A simplified proof of desingularization and applications. Rev. Mat. Iberoamericana 21 (2005), no. $2,349-458$. 
[6] Bierstone, E. And Milman, P.: Canonical desingularization in characteristic zero by blowing up the maximum strata of a local invariant. Invent. Math. 128 (1997), no. 7, 207-302.

[7] Bierstone, E. And Milman, P.: Desingularization algorithms I. Role of exceptional divisors. Moscow Math. J. 3 (2003), no. 3, 751-805, 1197.

[8] Cutkosky, S.: Resolution of singularities. Graduate Studies in Mathematics 63. Am. Math. Soc., Providence, RI, 2004.

[9] Encinas, S. And Hauser, H.: Strong resolution of singularities in characteristic zero. Comment. Math. Helv. 77 (2002), no. 4, 821-845.

[10] Encinas, S., Nobile, A. and Villamayor, O.: On algorithmic equiresolution and stratification of Hilbert schemes. Proc. London Math. Soc. 86 (2003), no. 3, 607-648.

[11] Encinas, S. And Villamayor, O.: A course on constructive desingularization and equivariance. In Resolution of singularities (Obergurl, 1997), 147-227. Progr. Math. 181. Birkhauser, Basel, 2000.

[12] Giraud, J.: Sur la théorie du contact maximal. Mat. Z. 137 (1974), 285-310.

[13] Hartshorne, R.: Algebraic geometry. Graduate Texts in Mathematics 52. Springer-Verlag, New York-Heidelberg, 1977.

[14] Hironaka, H.: Resolution of singularities of an algebraic variety over a field of characteristic zero, I-II. Ann. of Math. (2) $\mathbf{7 9}$ (1964), 109-326.

[15] Hironaka, H.: Idealistic exponent of singularity. In Algebraic geometry (J.J. Sylvester Sympos., John Hopkins Univ., Baltimore, Md., 1976), 52125. Johns Hopkins Univ. Press, Baltimore, Md., 1977.

[16] Kollar, J.: Resolution of singularities-Seattle lecture. Preprint, arXiv: math. AG/0508332v1.

[17] Kollar, J.: Lectures on resolution of singularities. Annals of Mathematics Studies 166. Princeton University Press, Princeton, NJ, 2007.

[18] Kunz, E.: Introduction to Commutative Algebra and Algebraic Geometry. Birkhauser Boston, Boston, MA, 1985.

[19] Matsumura, H.: Commutative Algebra. W.A. Benjamin, New York, 1970.

[20] Matsumura, H.: Commutative ring theory. Cambridge Studies in Advanced Mathematics 8. Cambridge University Press, Cambridge, 1989.

[21] Matsuki, K.: Notes on the inductive algorithm of resolution of singularities of S. Encinas and O. Villamayor. Preprint, arXiv:math.AG/0103120.

[22] Mumford, D.: Lectures on curves on an algebraic surface. Annals of Mathematics Studies 59. Princeton University Press, Princeton, NJ, 1966.

[23] Nobile, A.: A note on flat algebras. Proc. Amer. Math. Soc. 64 (1977), no. 2, 206-208.

[24] Schlessinger, M.: Functors of Artin rings. Trans. Amer. Math. Soc. 130 (1968), 208-222. 
[25] Teissier, B.: Résolution simultanée. In Séminaire sur les sigularités des surfaces., 71-81. Lecture Notes in Mathematics 777. Springer, Berlin, 1980.

[26] Villamayor, O.: Constructiveness of Hironaka's resolution. Ann. Sci. École Norm. Sup. (4) 22 (1989), no. 1, 1-32.

[27] Villamayor, O.: Patching local uniformizations. Ann. Sci. École Norm. Sup. (4) 25 (1992), no. 6, 629-677.

[28] Wlodarczyk, J.: Simple Hironaka resolution in characteristic zero. J. Amer. Math. Soc. 18 (2005), no. 4, 779-822.

[29] Zariski, O.: Studies in equisingularity, I. Amer. J. Math. 87 (1965), $507-536$.

[30] Zariski, O.: Studies in equisingularity, II. Amer. J. Math. 87 (1965), 972-1006.

Recibido: 23 de enero de 2008

Revisado: 21 de abril de 2008

Augusto Nobile

Louisiana State University

Department of Mathematics

Baton Rouge, LA 70803, USA

nobile@math.lsu.edu 